\title{
A Sufficient Condition for the Existence and the Uniqueness of Smooth Solutions to Boundary Value Problems for Elliptic Systems*
}

By

Nobuhisa IwASAKI

\section{§1. Introduction, Problem and Result}

In this paper we shall give a sufficient condition for boundary value problems of elliptic systems to have the unique infinitely differentiable solution for any infinitely differentiable data function. We shall consider an elliptic partial differential system given in $\operatorname{ADN}[2]$, but it includes a real parameter. We give some notations before we state the problem we shall deal with. Let $\Omega$ be a bounded open set in $R^{n+1}(n \geqq 1)$ with an infinitely differentiable boundary $\partial \Omega$. $\operatorname{Pol}(k)$ are spaces of polynomials of degree $\leqq k$ in $(\xi, \lambda) \in R^{n+1} \times R$ with coefficients of infinitely differentiable functions on $\bar{\Omega}$ if $k \geqq 0$ and $\operatorname{Pol}(k)=\{0\}$ if $k<0$, where $k$ are integers. $\mathscr{A}=\left(a_{i j}\right)_{\substack{1 \leqq i \leqq m \\ 1 \leqq j \leqq m}}$ and $\mathscr{B}=\left(b_{i j}\right)_{\substack{1 \leqq i \leqq l \\ 1 \leqq j \leqq m}}$ are systems of polynomials such that $a_{i j} \in \operatorname{Pol}\left(r_{j}+s_{i}\right)$ and $b_{i j} \in \operatorname{Pol}\left(r_{j}+t_{i}\right)$, where $\left(r_{i}\right),\left(s_{i}\right)$ and $\left(t_{i}\right)$ are systems of integers, which we call weight indices of $(\mathscr{A}, \mathscr{B}) . \quad \mathscr{A}^{0}=\left(a_{i j}^{0}\right)$ and $\mathscr{B}^{0}=\left(b_{i j}^{0}\right)$ are principal parts of $\mathscr{A}$ and $\mathscr{B}$, respectively, that is, $a_{i j}^{0}\left(b_{i j}^{0}\right)$ be homogeneous parts of order $r_{j}+s_{i}\left(r_{j}+t_{i}\right)$ of $a_{i j}\left(b_{i j}\right) . A=\left(A_{i j}\right)$ is the partial differential system (with a parameter $\lambda)$ on $C_{m}^{\infty}(\bar{\Omega}) \quad\left(\mathbb{C}^{m}\right.$-valued infinitely differentiable functions on $\bar{\Omega})$ and $B=\left(B_{i j}\right)$ the trace operator by partial differential operators from $C_{m}^{\infty}(\bar{\Omega})$ to $C_{l}^{\infty}(\partial \Omega)$, that is, they operate to $e=\left(e_{j}\right)$, elements of $C_{m}^{\infty}(\bar{\Omega})$, such that

Received October 23, 1974.

* Thesis presented to Kyoto University. 


$$
\begin{aligned}
& \left\{\begin{array}{l}
A e=f=\left(f_{i}\right) \in C_{m}^{\infty}(\bar{\Omega}), \quad \sum_{j=1}^{m} A_{i j} e_{j}=f_{i} \quad \text { and } \\
A_{i j} e_{j}=a_{i j}\left(\partial_{x}, \lambda\right) e_{j}
\end{array}\right. \\
& \left\{\begin{array}{l}
B e=g=\left(g_{i}\right) \in C_{l}^{\infty}(\partial \Omega),\left.\quad \sum_{j=1}^{m} B_{i j} e_{j}\right|_{\partial \Omega}=g_{i} \quad \text { and } \\
B_{i j} e_{j}=b_{i j}\left(\partial_{x}, \lambda\right) e_{j},
\end{array}\right.
\end{aligned}
$$

where $\partial_{x}=\left(i^{-1} \frac{\partial}{\partial x_{j}}\right)$.

We consider a boundary value problem,

$$
\begin{cases}A u=f & \text { on } \Omega \\ B u=g & \text { on } \partial \Omega\end{cases}
$$

where $f \in C_{m}^{\infty}(\bar{\Omega})$ and $g \in C_{l}^{\infty}(\partial \Omega)$ are data functions and $u \in C_{m}^{\infty}(\bar{\Omega})$ is an unknown function. If $A$ is of type $\operatorname{ADN}$ and if $(A, B)$ is coercive, we know that the solutions to (1.1) belong to $C_{m}^{\infty}(\bar{\Omega})$ and the operator $(A, B)$ has an index, but we have so far ignored the existence and the uniqueness in general. On the other hand if $A$ is of Garding type or of symmetric type and if some relations between $A$ and $B$ are assumed, we have the theorem of existence and uniqueness on some Sobolev spaces for sufficiently large $\lambda$ by the use of variational method, but $C^{\infty}$-regularity up to the boundary does not generally hold.

In Agmon [1] and Lions-Magenes [11] we can find a non-variational case where existence and uniqueness are shown. Extending it and introducing a more general condition for regularity than coerciveness we single out a class of boundary value problem, for which regularity, uniqueness and existence of solution are guaranteed. We state these as the following theorem which is our main result. Some terms in the theorem will be defined later. Roughly speaking the condition described in the theorem is one of sufficient conditions under which the pseudo-differential operator defined by the Lopatinsky matrix of ( $A$, $B)$ on the boundary is solvable and hypoelliptic.

Theorem 1. If $(A, B)$ is an elliptic system properly linked by $\lambda$ on $\Omega$, then there exists a constant $\lambda_{0}$ such that $(A, B)$ is an isomor- 
phism from $C_{m}^{\infty}(\bar{\Omega})$ to $C_{m}^{\infty}(\bar{\Omega}) \times C_{l}^{\infty}(\partial \Omega)$ for $\lambda \geqq \lambda_{0}$.

Corollary of Theorem 1 . If $(A, B)$ is an elliptic system strongly linked by $\lambda$ on $\bar{\Omega}$, then $\operatorname{Index}(A, B)=\operatorname{dim}$ of $\operatorname{coker}(A, B)-\operatorname{dim}$ of $\operatorname{ker}(A, B)=0$ for any $\lambda \in \mathbb{C}^{1}$.

ADN [2] says that these types of partial differential systems ( $A$, $B)$ can be remodeled systematically to equivalent systems $\left(A^{\prime}, B^{\prime}\right)$ such that order of each term of $A^{\prime} \leqq 1$ and of $B^{\prime} \leqq 0$. So, we may assume that $(A, B)$ is the one remodeled. The main reason why we remodel it is that we embed the bundle attached to the principal symbol of the partial differential system $A$ and complemented by the trace operator $B$ into a trivial vector bundle on $T^{*}(\bar{\Omega})$. We explain here only about equivalence we have used above. We call two partial differential systems $P_{0}$ and $P_{1}$ equivalent if there exist six partial differential systems $F_{i}$, $G_{i}$ and $Q_{i}(i=0,1)$ such that

$$
\left(\begin{array}{c}
Q_{0}, P_{0} \\
G_{0}, F_{0}
\end{array}\right)\left(\begin{array}{c}
Q_{1}, P_{1} \\
G_{1}, F_{1}
\end{array}\right)=\left(\begin{array}{c}
Q_{1}, P_{1} \\
G_{1}, F_{1}
\end{array}\right)\left(\begin{array}{c}
Q_{0}, P_{0} \\
G_{0}, F_{0}
\end{array}\right)=I
$$

In our case we have $P_{0}=\left(\begin{array}{c}A \\ B\end{array}\right)$ and $P_{1}=\left(\begin{array}{c}A^{\prime} \\ B^{\prime}\end{array}\right)$.

Let $\Omega=R_{+}^{n+1}$ and let us put $x=\left(x_{i}\right)_{i=1, \ldots, n}, y=x_{n+1}, \xi=\left(\xi_{i}\right)_{i=1, \ldots, n}$, and $\mu=i \eta=i \xi_{n+1}$. We define $\mathscr{N}_{i}(\mu)$ as following, where $\left(r_{j}\right),\left(s_{j}\right)$ and $\left(t_{j}\right)$ are the systems of weight indices of $(\mathscr{A}, \mathscr{B}), m$ the degree of square matrix $\mathscr{A}$ and $r_{0}=\max _{j}\left(r_{j}\right)$.

$$
\begin{aligned}
& \mathscr{N}_{0}(\mu)=\left((1-\eta)^{r_{j}-r_{0}} \delta_{i j}\right) \\
& \mathscr{N}_{1}(\mu)=\left((1-\eta)^{s_{j}+r_{0}-1} \delta_{i j}\right)
\end{aligned}
$$

and $\left(\delta_{i j}\right)$ is Kronecker's $\delta$.

Definition 1.1. When we assume that $\mathscr{A}^{0}$ is non-singular for all real vector $(\eta, \xi, \lambda) \neq 0$ at $(x, y)=0$, we can define the followings.

$$
\mathscr{P}=(2 \pi i)^{-1} \int_{\Gamma} \mathscr{N}_{0}(\mu) \mathscr{A}^{0^{-1}}(\mu) \mathscr{N}_{1}(\mu) \mathscr{A}_{0}^{0} d \mu,
$$




$$
\mathscr{D}=(2 \pi i)^{-1} \int_{\Gamma} \mathscr{B}^{0} \mathscr{A}^{0^{-1}}(\mu) \mathscr{N}_{1}(\mu) \mathscr{A}_{0}^{0} d \mu
$$

where $|\xi|^{2}+\lambda^{2}=1, \mathscr{A}^{0}$ and $\mathscr{B}^{0}$ are the principal parts of $\mathscr{A}$ and $\mathscr{B}, \mathscr{A}_{0}^{0}=\left.\mathscr{A}^{0}(1)\right|_{(\xi, \lambda)=0}$ and $\Gamma$ is a $C^{\infty}$-Jordan contour which lies in $\mathbb{C}_{-}$ (left half plane) and encloses the roots of $\operatorname{det} \mathscr{A}^{0}(\mu)=0$ with negative real parts.

$$
\begin{aligned}
& \left.\begin{array}{l}
\mathscr{P}(\zeta)=\mathscr{P}(\zeta /|\zeta|) \\
\mathscr{D}(\zeta)=\mathscr{D}(\zeta /|\zeta|)
\end{array}\right\} \text { when } \zeta=(\xi, \lambda) \neq 0 \\
& \left.\begin{array}{l}
\mathscr{H}_{\alpha \beta}^{1}=\partial_{x}^{\alpha} \partial_{\xi}^{\beta} \mathscr{D} \cdot \mathscr{P} \\
\mathscr{H}_{\alpha \beta}^{2}=\mathscr{P}^{*} \partial_{x}^{\alpha} \partial_{\xi}^{\beta} \mathscr{D}^{*}
\end{array}\right\} \text { when } \zeta=(\xi, \lambda) \neq 0
\end{aligned}
$$

where $\mathscr{P}^{*}$ and $\mathscr{D}^{*}$ are adjoint matrices of $\mathscr{P}$ as the operator on $\mathbb{C}^{m}$ and of $\mathscr{D}$ as the operator from $\mathbb{C}^{m}$ to $\mathbb{C}^{l}$.

Definition 1.2. 1) We call a system $A$ a favourably elliptic system (at $(x, y)=0)$ if $\mathscr{A}^{0}$ is non-singular at $(x, y)=0$ for any real vector $(\eta, \xi, \lambda) \neq 0$.

2) We call an elliptic system $(A, B)$ linked by $\lambda$ if it satisfies the three properties that (1) $A$ is favourably elliptic, (2) the degree of $A$ (=the degree of $\left.\operatorname{det} \mathscr{A}^{0}\right)=2 l$ and (3) dim of range $\mathscr{D}=l$ if $\lambda>0$.

3) We call an elliptic system $(A, B)$ properly linked by $\lambda$ if it is linked by $\lambda$ and if there exist constants $c$ such that

$$
\begin{aligned}
& \lambda|\mathscr{P} f| \leqq c|\mathscr{D} f| \\
& \left|\mathscr{H}_{\alpha \beta}^{1} f\right|^{2} \leqq c|\mathscr{D} f| \\
& \left|\mathscr{H}_{\alpha \beta}^{2} g\right|^{2} \leqq c\left|\mathscr{D}^{*} g\right|
\end{aligned}
$$

for $\lambda \geqq 0,|\zeta|=1,|f|=1\left(f \in \mathbb{C}^{m}\right),|g|=1\left(g \in \mathbb{C}^{l}\right)$ and $|\alpha|+|\beta|=1$ at a neighborhood of $(0,0)$.

4) We call an elliptic system $(A, B)$ strongly linked by $\lambda$ if it is linked by $\lambda$ and if $\operatorname{dim}$ of range $\mathscr{D}=l$ on $\lambda \geqq 0$.

Definition 1.3. We call an elliptic system $(A, B)$ properly (strongly) 
linked by $\lambda$ on $\bar{\Omega}$ if $A$ is a favourably elliptic system at each point of $\bar{\Omega}$ and if $(A, B)$ is properly (strongly) linked by $\lambda$ at each point of the boundary $\partial \Omega$ with respect to a coordinate function on $\bar{\Omega},(n-1)$ of which is a coordinate function on $\partial \Omega$.

\section{Example.}

$$
\left\{\begin{array}{l}
\left(-\Delta+\lambda^{2}\right) u=f \quad \text { on } \quad \Omega \\
\left(\varphi \frac{\partial}{\partial v}+\lambda\right) u=g \quad \text { on } \partial \Omega
\end{array}\right.
$$

where $\varphi$ is a non-negative $C^{\infty}$-function on $\partial \Omega$ and $v$ is the conormal. Then, $\|u\|_{s+1, \Omega} \leqq c_{s}(\lambda)\left\{\|f\|_{s, \Omega}+\|g\|_{s+\frac{1}{2}, \partial \Omega}\right\}$ for sufficiently large $\lambda$.

We shall study a special case by the method of pseudo-differential operators and we shall obtain results sufficient to show Theorem 1 in general cases by regarding them as small perturbations of the special case. It is as follows.

The domain $\Omega$ is $R_{+}^{n+1}$. The equation is (1.10).

$$
\left\{\begin{array}{l}
\frac{\partial}{\partial y}+M\left(x, \partial_{x}, \lambda\right) u=f \\
\left.B\left(x, \partial_{x}, \lambda\right) u\right|_{y=0}=g
\end{array}\right.
$$

where $M\left(x, \partial_{x}, \lambda\right)$ and $B\left(x, \partial_{x}, \lambda\right)$ are pseudo-differential operators whose symbols are $M(x, \xi, \lambda)$ and $B(x, \xi, \lambda) . m \times m$ matrix $M(x, \xi, \lambda)$ and $l \times m$ matrix $B(x, \xi, \lambda)$ are $C^{\infty}$-functions, in real variable $(x, \xi, \lambda) \in R^{n}$ $\times\left\{R^{n} \times R-(0,0)\right\}$, which do not depend on $x$ out side of a bounded set in $x$-space, and functions of homogeneous order 1 and 0 in $(\xi, \lambda)$, respectively. We set that $\mathscr{A}=\mu+M(x, \xi, \lambda), \mathscr{B}=B(x, \xi, \lambda)$ and weight indices $r_{j}=1, s_{j}=0$ and $t_{j}=-1(j=1, \ldots, m$ or $l)$. Then, Definition $1.1 \sim 3$ are well defined.

Definition. Let us set $W_{b} \subset H_{m}^{0}\left(R_{+}^{n+1}\right) \times H_{m}^{-1 / 2}\left(R^{n}\right)$ as $(u, v) \in W_{b}$ if and only if $u,\left\{\frac{\partial}{\partial y}+M\left(x, \partial_{x}, \lambda\right)\right\} u \in H_{m}^{0}\left(R_{+}^{n+1}\right)$, (then, $u(\cdot, 0)$ is well defined in $\left.H_{m}^{-1 / 2}\left(R^{n}\right)\right), B\left(x, \partial_{x}, \lambda\right) v \in H_{l}^{1 / 2}\left(R^{n}\right)$ and $v=u(\cdot, 0)$.

Remark. $H_{m}^{s}(\Omega)$ is the Sobolev space of $\mathbf{C}^{m}$-valued distributions on $\Omega$. 
Definition. $C(\lambda)$ stands for the closed operator, from $H_{m}^{0}\left(R_{+}^{n+1}\right)$ $\times H_{m}^{-1 / 2}\left(R^{n}\right)$ to $H_{m}^{0}\left(R_{+}^{n+1}\right) \times H_{l}^{1 / 2}\left(R^{n}\right),\left(\begin{array}{cc}\frac{\partial}{\partial y}+M\left(x, \partial_{x}, \lambda\right), & 0 \\ 0 & , B\left(x, \partial_{x}, \lambda\right)\end{array}\right)$ whose definition domain is $W_{b}$.

Theorem. Let us assume that the pseudo-differential system $\left(\frac{\partial}{\partial y}\right.$ $\left.+M\left(x, \partial_{x}, \lambda\right), B\left(x, \partial_{x}, \lambda\right)\right)$ is an elliptic system properly linked by $\lambda$ on $R_{+}^{n+1}$. Then we have:

1) There exists a constant $\lambda_{0}$ such that, if $\lambda \geqq \lambda_{0}, C(\lambda)$ has the inverse operator $R(\lambda)$, which is a bounded operator from $H_{m}^{0}\left(R_{+}^{n+1}\right) \times H_{l}^{1 / 2}\left(R^{n}\right)$ to $H_{m}^{0}\left(R_{+}^{n+1}\right) \times H_{m}^{-1 / 2}\left(R^{n}\right)$.

2) $R(\lambda)$ satisfies for non-negative integers $s$ the estimates that

$$
\|R(\lambda)\|_{s} \leqq c_{s} \hat{\lambda}^{-1}
$$

where $\|\cdot\|_{s}$ is the norm of bounded operators from $H_{m}^{s}\left(R_{+}^{n+1}\right) \times H_{l}^{s+1 / 2}\left(R^{n}\right)$ to $H_{m}^{s}\left(R_{+}^{n+1}\right) \times H_{m}^{s-1 / 2}\left(R^{n}\right)$, that we obtain when we induce the norms $\left(\left\|\left\{\frac{\partial}{\partial y}-\Lambda(\lambda)\right\}^{s} u\right\|^{2}+\left\|\Lambda^{t}(\lambda) v\right\|^{2}\right)^{1 / 2}$ in $H^{s} \cdot\left(R_{+}^{n+1}\right) \times H_{0}^{t}\left(R^{n}\right): \Lambda(\lambda)$ is the pseudodifferential operator with the symbol $\left(|\xi|^{2}+\lambda^{2}\right)^{1 / 2}$.

The results of Theorem are not enough to show regularity in general cases. We have to estimate $R(\lambda)$ more precisely to do so. It is possible for systems properly linked by $\lambda$.

Definition. Let $s \geqq 0$ and $i=0$ or 1 .

1) $Y_{i}^{s}(\lambda)=L^{2}\left(R_{+}^{1} ; H_{m}^{s}\left(R^{n}\right)\right) \times H_{\alpha_{i}}^{s-1 / 2+i}\left(R^{n}\right)$ with the norm $\|U\|_{Y_{i}^{s}(\lambda)}$ $=\left(\left\|\Lambda^{s}(\lambda) u\right\|^{2}+\left\|\Lambda^{s-1 / 2+i}(\lambda) v\right\|^{2}\right)^{1 / 2} ; U=(u, v) \in Y_{i}^{s}(\lambda)$, where $\alpha_{0}=m, \alpha_{1}=l$ and $L^{2}\left(R_{+}^{1} ; H_{m}^{s}\left(R^{n}\right)\right)$ is a space of $H_{m}^{s}\left(R^{n}\right)$-valued $L^{2}$-functions in $y \in(0$, $\infty)$.

2) Let $\chi=\left(\chi_{j}\right)_{j=0, \ldots, k}$ be a system of $C^{\infty}$-functions such that $\chi_{0} \equiv 1, \chi_{i} \equiv 1$ or $\in C_{0}^{\infty}\left(\overline{R_{+}^{n+1}}\right)$, and $\chi_{i+1}=\chi_{i} \chi_{i+1}(i \geqq 1)$.

3) $X_{i}^{s}(\lambda, \chi)$ stands for the closure of $C_{0}^{\infty}\left(\overline{R_{+}^{n+1}}\right) \times C_{0}^{\infty}\left(R^{n}\right)$ in the space $\left\{U ; \chi_{j} U \in Y_{i}^{\sigma_{j}}(\lambda), \sigma_{0}=0, \sigma_{j}=s-(k-j) / 2,1 \leqq j \leqq k\right\}$ with the semi-norms $p_{i j}(U)=\left\|\chi_{j} U\right\|_{Y_{i}^{\sigma_{j}(\lambda)}}$ and the norm $\|U\|_{X_{i}^{s}(\lambda, \chi)}=\left(\sum_{j=0}^{k} p_{i j}(U)^{2}\right)^{1 / 2}$, where $\chi_{j} U=\left(\chi_{j} u, \chi_{j}^{0} v\right) ; U=(u, v)$ and $\chi_{j}^{0}=\left.\chi_{j}\right|_{y=0}$. 
4) Let us set $q_{i j}=\left(\sum_{v=0}^{j} p_{i v}^{2}\right)^{1 / 2} ; 0 \leqq j \leqq k, q_{i-1}=0$.

Theorem. Let us assume that the pseudo-differential system $\left(\frac{\partial}{\partial y}\right.$ $\left.+M\left(x, \partial_{x}, \lambda\right), B\left(x, \partial_{x}, \lambda\right)\right)$ is an elliptic system properly linked by $\lambda$ on $R_{+}^{n+1}$. Then, there exists a constant $\lambda_{0}$ such that the inverse operator $R(\lambda)$ of $C(\lambda)$ in the previous Theorem is a bounded operator from $X_{1}^{s}(\lambda, \chi)$ to $X_{0}^{s}(\lambda, \chi)$ if $\lambda \geqq \lambda_{0}$ and $0 \leqq s \leqq k / 2$. It satisfies the following estimates for $U \in X_{1}^{s}(\lambda, \chi)$.

$$
p_{0 j}(R(\lambda) U) \leqq c_{0} \lambda^{-1} p_{1 j}(U)+c(s, \chi) \lambda^{-3 / 2} q_{1 j-1}(U),
$$

where $c_{0}$ is a constant not depending on $s \geqq 0, \lambda \geqq \lambda_{0}$ and $\chi$.

Remark. In this theorem it is important that the constant $c_{0}$ does not depend on $s, \lambda$ and $\chi$.

If the system $M$ and $B$ do not depend on the variable $x$, we can easily construct $R(\lambda)$ by Fourier transform. So, we consider what we obtain when we regard the variable $x$ in $M$ and $B$ as a parameter. It defines a pseudo-differential operator and gives a first approximation of $R(\lambda)$, that is, if we denote it by $R^{\prime}(\lambda)$, we obtain the relations that $R(\lambda) \cdot(I+S(\lambda))=R^{\prime}(\lambda)$ and $(I+T(\lambda)) \cdot R(\lambda)=R^{\prime}(\lambda)$. We can show that $R^{\prime}(\lambda), S(\lambda)$ and $T(\lambda)$ are defined by pseudo-differential operators that satisfy similar estimates as ones in the previous theorems if we assume the conditions in Definition 1.2. Thus, $I+S(\lambda)$ and $I+T(\lambda)$ are invertible.

We write down the results for pseudo-differential operators we use in proof of the theorems. $R^{\prime}, S$ and $T$ are defined by compositions of pseudo-differential operators to which we can apply them. They are due to the papers by A.P. Calderon and R. Vaillancourt [4] and L. Hörmander [8]. If we follow them carefully, we have all of them without other technique.

We consider pseudo-differential operators on the space of distributions valued in a Hilbert space $X$, that is, symbols of pseudo-differential operators are $C^{\infty}$-functions valued in $B L(X)$ the space of bounded linear operators on $X$. Let $C^{\infty}\left(R^{s}, X\right)$ and $C^{\infty}\left(R^{s}, B L(X)\right)$ stand for the space of $X$ and $B L(X)$-valued $C^{\infty}$-functions on $R^{s}$ with respect to the topologies 
by the norms of $X$ and $B L(X)$, respectively. We denote the norms of $X$ and $B L(X)$ by $|\cdot|$ and the canonical norm of $L^{2}\left(R^{s}, X\right)$ the space of $X$-valued square integrable functions by $\|\cdot\|$.

Let $p(x, \xi, \lambda)$ and $q(x, \xi, \lambda) \in C^{\infty}\left(R^{n} \times R^{n}, B L(X)\right)$ with a parameter $\lambda \in(0, \infty)$ and let us assume that $p(x, \xi, \lambda)$ and $q(x, \xi, \lambda)$ are independent of $x$ outside a ball and that for all multi-indices $\alpha, \beta$ there exist constants $c_{\alpha \beta}, m_{i}, \delta_{i}$, and $\rho_{i}(i=1,2)$ such that

$$
\begin{aligned}
& \left|p_{(\beta)}^{(\alpha)}(x, \zeta)\right| \leqq c_{\alpha \beta} \lambda^{-|\alpha|}(|\zeta| / \lambda)^{m_{1}+\delta_{1}|\beta|-\rho_{1}|\alpha|} \\
& \left|q_{(\beta)}^{(\alpha)}(x, \zeta)\right| \leqq c_{\alpha \beta} \lambda^{-|\alpha|}(|\zeta| / \lambda)^{m_{2}+\delta_{2}|\beta|-\rho_{2}|\alpha|}
\end{aligned}
$$

on $\zeta=(\xi, \lambda) \in R^{n} \times(0, \infty)$, where $p_{(\beta)}^{(\alpha)}(x, \zeta)=\partial_{\xi}^{\alpha} \partial_{x}^{\beta} p(x, \zeta)$.

Let us set that for $u \in C_{0}^{\infty}\left(R^{n}, X\right)$,

$$
K(\lambda) u=q(\partial, \lambda) p(\partial, \lambda) u-\sum_{|\alpha|<N}(i)^{|\alpha|}(\alpha !)^{-1} q^{(\alpha)}(\partial, \lambda) \circ p_{(\alpha)}(\partial, \lambda) u
$$

If $0 \leqq \delta_{1}<\rho_{2} \leqq 1$ and $0 \leqq \delta_{i} \leqq \rho_{i} \leqq 1$, then for any real number $s$ and integer $m$ there exists an integer $N_{0}$ such that, if $N \geqq N_{0}$ and $\lambda \geqq 1$,

$$
\left\|\Lambda^{s}(\lambda) p(\partial, \lambda) u\right\| \leqq c_{s} \lambda^{-m_{1}}\left\|\Lambda^{s+m_{1}}(\lambda) u\right\|
$$

and

$$
\left\|\Lambda^{s}(\lambda) K(\lambda) u\right\| \leqq c_{s N} \lambda^{-N+s-m+2}\left\|\Lambda^{m}(\lambda) u\right\|, \quad \text { for } \quad u \in C_{0}^{\infty}\left(R^{n}, X\right) .
$$

Let $Y$ be another Hilbert space densely contained in $X$ by a continuous injection. We assume further for $q(x, \zeta)$ to satisfy that there exists an integer $\alpha_{0}$ such that $q_{(\beta)}^{(\alpha)}(x, \zeta) \in B L(X, Y)$ (bounded linear operators from $X$ to $Y$ ) and

$$
\left|q_{(\beta)}^{(\alpha)}(x, \zeta)\right|_{X Y} \leqq c_{\alpha \beta \lambda}(1+|\xi|)^{m_{3}+\delta_{2}|\beta|-\rho_{2}|\alpha|}
$$

for all $|\alpha| \geqq \alpha_{0}$ and $|\beta| \geqq 0$, where $|\cdot|_{X Y}$ is the norm of $B L(X, Y)$. Then, there exists an integer $N_{0}$ for any integer $m$ such that

$$
\|K(\lambda) u\|_{Y} \leqq c_{m N \lambda}\left\|\Lambda^{m}(\lambda) u\right\|_{X} \quad \text { for } \quad N \geqq N_{0} \quad \text { and } \quad u \in C_{0}^{\infty}\left(R^{n}, X\right) \text {, }
$$

where $\|\cdot\|_{X}$ and $\|\cdot\|_{Y}$ stand for the norms of $L^{2}\left(R^{n}, X\right)$ and $L^{2}\left(R^{n}, Y\right)$, respectively. 


\section{§. Preliminary Analysis for Proof}

We consider the following $m \times m$ matrix $M(x, \xi, \lambda)$ such that $M(x$, $\xi, \lambda)$ is a $C^{\infty}$-function, in real variables $(x, \xi, \lambda) \in R^{n} \times\left\{R^{n} \times R^{1}-(0,0)\right\}$, which does not depend on $x$ out side of a bounded set in $x$-space, and a function of homogeneous order 1 in $(\xi, \lambda)$, that is, $\alpha M(x, \xi, \lambda)=M(x$, $\alpha \xi, \alpha \lambda)$ for all $\alpha>0$. We assume that $\mu+M(x, \xi, \lambda)$ is non-singular when $\mu=-i \eta$ and when $(\eta, \xi, \lambda)$ are non-zero real vectors, that is, there exists the inverse of $\mu+M(x, \xi, \lambda)$. From this assumption we are able to divide the eigenspace of $M(x, \xi, \lambda)$ into two parts as followings.

$$
\begin{aligned}
& P_{+}(x, \xi, \lambda)=(2 \pi i)^{-1} \int_{\Gamma_{+}}(\mu+M(x, \xi, \lambda))^{-1} d \mu \\
& P_{-}(x, \xi, \lambda)=(2 \pi i)^{-1} \int_{\Gamma_{-}}(\mu+M(x, \xi, \lambda))^{-1} d \mu
\end{aligned}
$$

where $\Gamma_{+}\left(\Gamma_{-}\right)$is a Jordan curve which is laid in the right (left) half plane of $\mathbf{C}$ and surrounds the eigenvalues of $-M(x, \xi, \lambda)$ of which the real parts are positive (negative). $P_{+}$and $P_{-}$are projections and satisfy the relations that $P_{ \pm}(x, \alpha \xi, \alpha \lambda)=P_{ \pm}(x, \xi, \lambda)$ for all $\alpha>0$ and that $P_{+}+P_{-}=I$. If we set $U(y)=e^{-M(x, \xi, \lambda) y} U(0)$ and $U(0)$ a vector, then $U(y)$ satisfies the equation that $\left\{\frac{\partial}{\partial y}+M(x, \xi, \lambda)\right\} U(y)=0$ and $U(y)$ increases (decreases) in the exponential order as $y \rightarrow+\infty$ if $U(0)$ belongs to the range of $P_{+}\left(P_{-}\right)$. We consider another $l \times m$ matrix $B(x, \xi, \lambda)$ such that $B(x, \xi, \lambda)$ is a $C^{\infty}$-function, in real variable $(x, \xi, \lambda) \in R^{n} \times\left\{R^{n}\right.$ $\left.\times R^{1}-(0,0)\right\}$, which does not depend on $x$ outside of a bounded set in $x$-space, and a function of homogeneous order 0 in $(\xi, \lambda)$, that is, $B(x, \xi, \lambda)=B(x, \alpha \xi, \alpha \lambda)$ for all $\alpha>0$.

<Assumption (A)> Relations between $M(x, \xi, \lambda)$ and $B(x, \xi, \lambda)$.

1) $\operatorname{dim}\left[\right.$ range of $\left.P_{-}(x, \xi, \lambda)\right]=l$.

2) When $\lambda>0,|\xi|^{2}+\lambda^{2}=1,|f|=1,|g|=1, f \in \mathbb{C}^{m}, g \in \mathbb{C}^{l}$ and $|\alpha|+|\beta|=1$, then there exist constants $c>0$ such that

a) $\quad \lambda\left|P_{-}(x, \xi, \lambda) f\right| \leqq c|D(x, \xi, \lambda) f|$ 
b) $\left|H_{\alpha \beta}^{1}(x, \xi, \lambda) f\right|^{2} \leqq c|D(x, \xi, \lambda) f|$

c) $\left|H_{\alpha \beta}^{2}(x, \xi, \lambda) g\right|^{2} \leqq c\left|D^{*}(x, \xi, \lambda) g\right|$,

where $D(x, \xi, \lambda) \equiv B(x, \xi, \lambda) P_{-}(x, \xi, \lambda), P_{-}^{*}$ and $D^{*}$ are adjoint matrices of $P_{-}$as the operator on $\mathbb{C}^{m}$ and of $D$ as the operator from $\mathbb{C}^{m}$ to $\mathbb{C}^{l}$, respectively, and

$$
\begin{aligned}
& H_{\alpha \beta}^{1}(x, \xi, \lambda) \equiv \partial_{x}^{\alpha} \partial_{\xi}^{\beta} D(x, \xi, \lambda) \cdot P_{-}(x, \xi, \lambda) \\
& H_{\alpha \beta}^{2}(x, \xi, \lambda) \equiv P_{-}^{*}(x, \xi, \lambda) \partial_{x}^{\alpha} \partial_{\xi}^{\beta} D^{*}(x, \xi, \lambda) .
\end{aligned}
$$

Under this <Assumption $(A)>$ we go on with our discussion. We denote the linear operator (matrix) $B(x, \xi, \lambda) \cdot P_{-}(x, \xi, \lambda)$ from range of $P_{-}(x, \xi, \lambda)$ to $\mathbb{C}^{l}$ by $D(x, \xi, \lambda)$, too. From the Assumption (A) 1) and 2) a), there exists the inverse operator (matrix) of $D(x, \xi, \lambda)$ when $\lambda>0$. We are going to estimate the operator-norm of the inverse operator in $(x, \xi, \lambda)$. Let $X \Xi \Lambda$ stand for the set $\left\{(x, \xi, \lambda) ;(x, \xi, \lambda) \in R^{2 n+1},|\xi|^{2}+\lambda^{2}\right.$ $=1, \lambda \geqq 0\}$. We first prove the following lemma.

Lemma 2.1. On $X \Xi \Lambda$ the following inequalities hold for a positive constant $c$.

$$
\begin{aligned}
& |D(x, \xi, \lambda) f| \geqq c \lambda^{1 / 2}\left|H_{\alpha \beta}^{1}(x, \xi, \lambda) f\right| \\
& \left|D^{*}(x, \xi, \lambda) g\right| \geqq c \lambda^{1 / 2}\left|H_{\alpha \beta}^{2}(x, \xi, \lambda) g\right|,
\end{aligned}
$$

where $|\alpha|+|\beta|=1$.

Proof. This lemma follows from the assumption (A) 2). Replacing $f$ in the inequalities of the assumption (A) 2$)$ by $P_{-}(x, \xi, \lambda) f / \| P_{-}(x, \xi$, $\lambda) f \mid$ and $g$ by $g /|g|$, where $\lambda \neq 0$, we obtain the following inequalities (2.2).

$$
\begin{aligned}
& \left|H_{\alpha \beta}^{1}(x, \xi, \lambda) f\right|^{2} \leqq c\left|P_{-}(x, \xi, \lambda) f\right||D(x, \xi, \lambda) f| \\
& \left|H_{\alpha \beta}^{2}(x, \xi, \lambda) g\right|^{2} \leqq c|g|\left|D^{*}(x, \xi, \lambda) f\right| ;|\alpha|+|\beta|=1 .
\end{aligned}
$$

Since we can apply to the above inequalities the assumption (A) 2) a), that is, $\lambda\left|P_{-}(x, \xi, \lambda) f\right| \leqq c|D(x, \xi, \lambda) f|$ and also $\lambda|g| \leqq c\left|D^{*}(x, \xi, \lambda) g\right|$, we obtain the inequalities which we have to prove.

End of proof. 
We now define the operator (matrix) $E(x, \xi, \lambda)$ for $\lambda>0$ by

$$
E(x, \xi, \lambda) \equiv D(x, \xi, \lambda)^{-1},
$$

where $D(x, \xi, \lambda)^{-1}$ means the inverse operator from $\mathbb{C}^{l}$ to the range of $P_{-}(x, \xi, \lambda)$ of $D(x, \xi, \lambda)$. It is clear that for $\lambda>0 E(x, \xi, \lambda)$ satisfies the following equalities.

$$
\begin{aligned}
& E(x, \xi, \lambda) \cdot D(x, \xi, \lambda)=P_{-}(x, \xi, \lambda) \\
& D(x, \xi, \lambda) \cdot E(x, \xi, \lambda)=I .
\end{aligned}
$$

Lemma 2.2. On $X \Xi \Lambda$ the following estimates hold for some positive constants $c_{\alpha \beta \gamma}$ and for all multi-indices $\alpha, \beta$ and $\gamma$.

1) $\left\|\partial_{x}^{\alpha} \partial_{\xi}^{\beta} \partial_{\lambda}^{\gamma} E(x, \xi, \lambda) \cdot D(x, \xi, \lambda)\right\| \leqq c_{\alpha \beta \gamma} \lambda^{-(|\alpha| / 2+|\beta| / 2+|\gamma|)}$

2) $\left\|D(x, \xi, \lambda) \cdot \partial_{x}^{\alpha} \partial_{\xi}^{\beta} \partial_{\lambda}^{\gamma} E(x, \xi, \lambda)\right\| \leqq c_{\alpha \beta \gamma} \lambda^{-(|\alpha| / 2+|\beta| / 2+|\gamma|)}$

3) $\left\|\partial_{x}^{\alpha} \partial_{\xi}^{\beta} \partial_{\lambda}^{\gamma} E(x, \xi, \lambda)\right\| \leqq c_{\alpha \beta \gamma} \lambda-(|\alpha| / 2+|\beta| / 2+|\gamma|+1)$.

Proof. We shall prove the lemma by maens of induction in the length $k=\sum_{i=1}^{n}\left(\alpha_{i}+\beta_{i}\right)+\gamma$ of multi-index $(\alpha, \beta, \gamma)$. If $k=0$, it is immediately proved from (2.3) and from the assumption (A) 2) a). We assume that the inequalities of lemma hold on $X \Xi \Lambda$ when $k \leqq k_{0}$. Let us differentiate the both sides of (2.3). By Leibniz formula we obtain that, denoting $\partial^{t}=\partial_{x}^{t_{\alpha}} \partial_{\xi_{\xi}}^{t_{\beta}} \partial_{\lambda^{\gamma}}^{t_{\nu}} ; t=\left(t_{\alpha}, t_{\beta}, t_{\gamma}\right)=p, q, r$ or $s$,

$$
\begin{aligned}
& \partial^{r}\{E \cdot D\}=\sum_{p+q=r} c_{p q} \partial^{p} E \cdot \partial^{q} D=\partial^{r} p_{-} \\
& \partial^{r}\{D \cdot E\}=\sum_{p+q=r} c_{p q} \partial^{q} D \cdot \partial^{p} E=0 ;|r|=k_{0}+1, \lambda>0 .
\end{aligned}
$$

Transposing terms except for $\partial^{s} E \cdot D$ and $D \cdot \partial^{s} E$ to the other side,

$$
\begin{gathered}
\partial^{r} E \cdot D=\partial^{r} P_{-}-\sum_{\substack{p+q=r \\
q \neq 0}} c_{p q} \partial^{p} E \cdot \partial^{q} D \\
=\partial^{r} P_{-} \cdot P_{-}-\sum_{\substack{p+q=r \\
q \neq 0}} c_{p q} \partial^{p} E \cdot \partial^{q} D \cdot P_{-} \\
D \cdot \partial^{r} E=-\sum_{\substack{p+q=r \\
q \neq 0}} c_{p q} \partial^{q} D \cdot \partial^{p} E
\end{gathered}
$$


because $D \cdot P_{-}=D$. We here notice that $P_{-}$and $D$ are infinitely differentiable on $X \Xi \Lambda$ and that, if $|r|=k_{0}+1, \partial^{p} E$ of the right hand sides of (2.4) and (2.5) satisfy the estimates of lemma by the assumption of induction because $|p| \leqq k_{0}$. From (2.4) and (2.5) we obtain (2.6) and (2.7), where $\kappa(t)=\left|t_{\alpha}\right| / 2+\left|t_{\beta}\right| / 2+t_{\gamma} ; t=\left(t_{\alpha}, t_{\beta}, t_{\gamma}\right)$.

$$
\begin{aligned}
& \partial^{r} E \cdot D=-\sum_{\substack{p+q=r \\
0<\kappa(q)<1}} c_{p q} \partial^{p} E \cdot \partial^{q} D \cdot P_{-}+F_{1} ; \\
& \left\|F_{1}(x, \xi, \lambda)\right\| \leqq c_{1}\left\{1+\sum_{\substack{p+q=r \\
\kappa(q) \geqq 1}} \lambda^{-(\kappa(p)+1)}\right\} \\
& D \cdot \partial^{r} E=-\sum_{\substack{p+q=r \\
0<\kappa(q)<1}} c_{p q} \partial^{q} D \cdot \partial^{p} E+F_{2} ; \\
& \left\|F_{2}(x, \xi, \lambda)\right\| \leqq c_{2}\left\{\sum_{\substack{p+q=r \\
\kappa(q) \geqq 1}} \lambda^{-(\kappa(p)+1)}\right\} .
\end{aligned}
$$

If $\kappa(q) \geqq 1$, then we have that $\kappa(p)+1=\kappa(r)+1-\kappa(q) \leqq \kappa(r)$. We next estimate the remained terms. Using (2.3),

$$
\partial^{p} E \cdot \partial^{q} D \cdot P_{-}=\left[\partial^{p} E \cdot D\right] \cdot\left[E \cdot \partial^{q} D \cdot P_{-}\right]
$$

Since $D^{*}$ and $P_{-}^{*} \partial^{q} D^{*}$ satisfy the estimate of Lemma 2.1, we replace $g$ in those inequalities with $E^{*}$ which is the adjoint operator of $E$ and we consider its adjoint. Then we obtain that, if $\kappa(q)<1$,

$$
\left\|E(x, \xi, \lambda) \cdot \partial^{q} D(x, \xi, \lambda) \cdot P_{-}(x, \xi, \lambda)\right\| \leqq c_{3} \lambda^{-\kappa(q)} .
$$

On the other hand $\left[\partial^{p} E(x, \xi, \lambda) \cdot D(x, \xi, \lambda)\right]$ satisfies the estimate 1) of this lemma by the assumption of induction because $|p| \leqq k_{0}$. Combining (2.9) and the above, we can estimate the remained terms of (2.6) and we have a bound $c_{4} \lambda^{-(\kappa(p)+\kappa(q))}$. Thus, the estimate 1) holds if $|r|=$ $k_{0}+1$. Since $E=P_{-} \cdot E$, we differentiate both sides of this equality,

$$
\begin{aligned}
\partial^{p} E & =\sum_{s+t=p} c_{s t} \partial^{s} P_{-} \cdot \partial^{t} E \\
& =P_{-} \cdot \partial^{p} E+\sum_{\substack{s+t=p \\
s \neq 0}} c_{s t} \partial^{s} P_{-} \cdot \partial^{t} E \\
& =P_{-} \cdot \partial^{p} E+F_{3}
\end{aligned}
$$




$$
\left\|F_{3}(x, \xi, \lambda)\right\| \leqq c_{5} \lambda^{-\kappa(p+q)} \quad \text { if } \quad|p| \leqq k_{0} \quad \text { and } \quad q \neq 0
$$

Substituting (2.10) for $\partial^{p} E$ in (2.7)

$$
\begin{gathered}
\sum_{\substack{p+q=r \\
0<\kappa(q)<1}} c_{p q} \partial^{q} D \cdot \partial^{p} E=\sum_{\substack{p+q=r \\
0<\kappa(q)<1}} c_{p q} \partial^{q} D \cdot P_{-} \cdot \partial^{p} E+F_{4} \\
\left\|F_{4}(x, \xi, \lambda)\right\| \leqq c_{6} \lambda^{-\kappa(r)} .
\end{gathered}
$$

Since $\partial^{q} D \cdot P_{-}=H_{q}^{1}$ if $0<\kappa(q)<1$, we have that

$$
\sum_{\substack{p+q=r \\ 0<\kappa(q)<1}} c_{p q} \partial^{q} D \cdot \partial^{p} E=\sum_{\substack{p+q=r \\ 0<\kappa(q)<1}} c_{p q}\left[H_{q}^{1} \cdot E\right] \cdot\left[D \cdot \partial^{p} E\right]+F_{4} .
$$

Since $D$ and $H_{q}^{1}$ satisfy the estimate of Lemma 2.1, we substitute $E$ for $f$ in those inequalities. We obtain that, if $\kappa(q)<1$,

$$
\left\|H_{q}^{1}(x, \xi, \lambda) \cdot E(x, \xi, \lambda)\right\| \leqq c_{7} \lambda^{-\kappa(q)} .
$$

On the other hand $\left[D(x, \xi, \lambda) \cdot \partial^{p} E(x, \xi, \lambda)\right]$ satisfies the inequality 2) of this lemma by the assumption of induction because $|p| \leqq k_{0}$. Combining these facts, we can estimate the remained terms of (2.7) and we have a bound $c_{8} \lambda^{-(\kappa(p)+\kappa(q))}=c_{8} \lambda^{-\kappa(r)}$. Thus, we obtain the inequality 2) when $|r|=k_{0}+1$. The inequality 3 ) is immediately obtained by operating $E$ to the right hand side of $\partial^{r} E \cdot D$ and by estimating it by the inequality 1), or by operating $E$ to the left hand side of $D \cdot \partial^{r} E$, by using (2.10) and by estimating it by the inequality 2) and the inequality 3) for $|p| \leqq k_{0}$.

End of proof.

\section{Lemma 2.3.}

1) $\left\|\partial^{p}\left[\partial^{q} E(x, \xi, \lambda) \cdot D(x, \xi, \lambda)\right]\right\|<c_{p q} \lambda^{-\kappa(p+q)}$.

2) $\left\|\partial^{p}\left[D(x, \xi, \lambda) \cdot \partial^{q} E(x, \xi, \lambda)\right]\right\|<c_{p q} \lambda^{-\kappa(p+q)}$.

Proof. We have only to prove the estimate 1) for $\partial^{p}\left[\partial^{q} E \cdot D\right] \cdot P_{-}$.

$$
\begin{aligned}
\partial^{p}\left[\partial^{q} E \cdot D\right] \cdot P_{-} & =\sum_{p=r+s} c_{r s} \partial^{r+q} E \cdot \partial^{s} D \cdot P_{-} \\
& =\sum_{\substack{p=r+s \\
\kappa(s)<1}} c_{r s} \partial^{r+q} E \cdot D \cdot E \cdot \partial^{s} D \cdot P_{-}+F_{1}
\end{aligned}
$$




$$
\left\|F_{1}(x, \xi, \lambda)\right\|<c \lambda^{-\kappa(p+q)}
$$

By Lemma 2.1 and 1) of Lemma 2.2 the remained terms are estimated with the bound $c \lambda^{-\kappa(r+q)-\kappa(s)}=c \lambda^{-\kappa(p+q)}$. Thus, we obtains 1$)$.

$$
\partial^{P}\left[D \cdot \partial^{q} E\right]=D \cdot \partial^{p+q} E+\sum_{\substack{r+s=p \\ 0<\kappa(r)<1}} c_{r s}\left[H_{r}^{1} \cdot E\right] \cdot\left[D \cdot \partial^{q+s} E\right]+F_{2},
$$

where $\left\|F_{2}(x, \xi, \lambda)\right\| \leqq c \lambda^{-\kappa(p+q)}$. From the above we obtain 2) by using Lemma 2.1 and 2) of Lemma 2.2.

End of proof.

Lemma 2.4. Let us define $J_{\alpha}^{1} \equiv \partial_{\xi}^{\alpha} E \cdot \partial_{x}^{\alpha} D$ and $J_{\alpha}^{2} \equiv \partial_{\xi}^{\alpha} D \cdot \partial_{x}^{\alpha} E$. On $X \Xi \Lambda$ for some positive constants $c_{\alpha \beta}$ and all multi-indices $p$ it holds that

1) when $|\alpha|=1,\left\|\partial^{p} J_{\alpha}^{1}(x, \xi, \lambda)\right\|<c_{\alpha p} \lambda^{-\kappa(p)-1}$

when $|\alpha| \geqq 2,\left\|\partial^{p} J_{\alpha}^{1}(x, \xi, \lambda)\right\|<c_{\alpha p} \lambda^{-\kappa(p)-|\alpha| / 2}$

2) when $|\alpha|=1,\left\|\partial^{p} J_{\alpha}^{2}(x, \xi, \lambda)\right\|<c_{\alpha p} \lambda^{-\kappa(p)-1}$

when $|\alpha| \geqq 2,\left\|\partial^{p} J_{\alpha}^{2}(x, \xi, \lambda)\right\|<c_{\alpha p} \lambda^{-\kappa(p)-|\alpha| / 2}$

where $\kappa(p)=\left|p_{1}\right| / 2+\left|p_{2}\right| / 2+\left|p_{3}\right| ; \partial^{p}=\partial_{x}^{p_{1}} \partial_{\xi}^{p_{2}} \partial_{\lambda}^{p_{3}}$.

Proof. We obtain the following equalities in the similar way as in the previous lemma.

$$
\begin{aligned}
& \partial_{\xi}^{\alpha} E \cdot \partial_{x}^{\alpha} D=-\left[\partial_{\xi}^{\alpha} E \cdot D\right] \cdot\left[\partial_{x}^{\alpha} E \cdot D\right]+\left[\partial_{\xi}^{\alpha} E \cdot D\right] \cdot \partial_{x}^{\alpha} P- \\
& \text { when }|\alpha|=1 \text {. } \\
& \partial_{\xi}^{\alpha} D \cdot \partial_{x}^{\alpha} E=-\left[D \cdot \partial_{\xi}^{\alpha} E\right] \cdot\left[D \cdot \partial_{x}^{\alpha} E\right]+\partial_{\xi}^{\alpha} D \cdot \partial_{x}^{\alpha} P_{-} \cdot E \\
& \text { when }|\alpha|=1 \text {. } \\
& \partial_{\xi}^{\alpha} D \cdot \partial_{x}^{\alpha} E=-\left[D \cdot \partial_{\xi}^{\alpha} E\right] \cdot\left[D \cdot \partial_{x}^{\alpha} E\right]+\partial_{\xi}^{\alpha} D \cdot \partial_{x}^{\alpha} P_{-} \cdot E \\
& +\sum_{\substack{s+t=\alpha \\
|s|=|t|=1}} c_{s t}\left\{\left[D \cdot \partial_{\xi}^{s} E\right] \cdot\left[D \cdot \partial_{\xi}^{t} E\right] \cdot\left[D \cdot \partial_{x}^{\alpha} E\right]\right. \\
& \left.-\partial_{\xi}^{s} D \cdot \partial_{\xi}^{t} P_{-} \cdot P_{-} \cdot \partial_{x}^{\alpha} E+\partial_{\xi}^{\alpha} D \cdot \partial_{x}^{s} P_{-} \cdot \partial_{x}^{t} E\right\} \\
& \text { when }|\alpha|=2 \text {. }
\end{aligned}
$$


When $|\alpha|=1$, we apply Lemma 2.3 and 3) of Lemma 2.2 to the right hand sides of (2.11) and (2.12) and these derivatives by $\partial^{p}$. We obtain the estimates. When $|\alpha|=2$, we apply same ones to the right hand sides of (2.13) and these derivatives by $\partial^{p}$. We obtain the estimates 2). The other cases are obtained by applying 3) of Lemma 2.2 to the defined forms of $J_{\alpha}^{i}$.

End of proof.

Lemma 2.5. Let $F(x, \xi, \lambda)$ be a matrix valued $C^{\infty}$-function in ( $x$, $\xi, \lambda) \in R^{2 n+1} \cap\{\lambda>0\}$, homogeneous of order $m$ in $\zeta=(\xi, \lambda)$. If $F(x, \xi$, $\lambda$ ) satisfies the following estimates on $X \Xi \Lambda=\left\{(x, \xi, \lambda) ;|\xi|^{2}+\lambda^{2}=1, \lambda \geqq 0\right\}$

$$
\left\|\partial^{p} F(x, \xi, \lambda)\right\| \leqq c_{p} \lambda^{-\left(\delta\left|p_{1}\right|+\varepsilon\left|p_{2}\right|+\left|p_{3}\right|+1\right)} ; \partial^{p}=\partial_{x}^{p_{1}} \partial_{\xi}^{p_{2}} \partial_{\lambda}^{p_{3}},
$$

then, $F(x, \xi, \lambda)$ satisfies the following estimates on the whole defined domain $R^{2 n+1} \cap\{\lambda>0\}$.

$$
\left\|\partial_{x}^{\alpha} \partial_{\xi}^{\beta} F(x, \xi, \lambda)\right\| \leqq c_{\alpha \beta} \lambda^{-(\delta|\alpha|+\varepsilon|\beta|+1)}|\zeta|^{(\delta|\alpha|+(\varepsilon-1)|\beta|+1+m)}
$$

(Note)

$f(\sigma)$ : function in $\sigma \in R^{m}$

$\psi(\xi)=\left(\psi_{i}(\xi)\right)_{i=1, \ldots, m}: R^{m}$-valued function in $\xi \in R^{l}$

$\beta=\left(\beta_{j}\right), \quad \tau=\left(\tau_{j}\right), \quad \omega=\left(\omega_{j}\right), \quad v=\left(v_{i}\right), \quad \mu=\left(\mu_{i j}\right)$

$i=1, \ldots, m, \quad j=1, \ldots, l$

$\beta_{j}, \tau_{j}, \omega_{j}, v_{i}, \mu_{i j}:$ non-negative integers

$$
\begin{aligned}
& \omega+\tau=\left(\omega_{j}+\tau_{j}\right), \quad|\mu|=\left(\mu_{i}^{(1)}\right) ; \mu_{i}^{(1)}=\sum_{j=1}^{l} \mu_{i j}, \quad \underline{\mu}=\left(\mu_{j}^{(2)}\right) ; \\
& \mu_{j}^{(2)}=\sum_{i=1}^{m} \mu_{i j} \\
& \partial_{\xi}^{\beta}=\partial_{\xi_{1}}^{\beta_{1}} \partial_{\xi_{2}}^{\beta_{2}} \cdots \partial_{\xi_{l}}^{\beta_{l}}, \partial_{\xi} \psi=\left(\psi_{i j}\right) ; \psi_{i j}=\partial_{\xi_{j}} \psi_{i} \\
& \left(\partial_{\xi} \psi\right)^{\mu}=\prod_{j=1}^{l} \prod_{i=1}^{m}\left(\psi_{i j}\right)^{\mu_{i j}} .
\end{aligned}
$$

With these notations we have easily the following formula, 


$$
\partial_{\xi}^{\beta} f(\psi(\xi))=\sum_{\substack{\omega=\underline{\mu} \\ v=\underline{\mu} \\ \nu+\tau=\beta}} c_{\mu \omega \beta}\left(\partial_{\psi}^{v} f\right)(\psi) \cdot \partial_{\xi}^{\tau}\left[\left(\partial_{\xi} \psi\right)^{\mu}\right]
$$

Proof. We first prove the lemma when $F$ is a homogeneous function of order 0 in $\zeta=(\xi, \lambda)$, i.e. $F(x, \zeta)=F(x, \zeta /|\zeta|)$. We use the notation of note.

$$
\partial_{\xi}^{\beta} F_{(\alpha)}(x, \psi(\zeta))=\sum_{\substack{\omega=\bar{\omega} \\ \nu=|\mu| \\ \omega+\tau=\beta}} c_{\mu \omega \beta} \partial_{\psi}^{v} F_{(\alpha)}(x, \psi(\zeta)) \cdot \partial_{\xi}^{\tau}\left[\left(\partial_{\xi} \psi\right)^{\mu}\right]
$$

where $l=n, m=n+1, \zeta=(\xi, \lambda), F_{(\alpha)}(x, \zeta)=\partial_{x}^{\alpha} F(x, \zeta)$,

$$
\psi_{i}(\zeta)=\xi_{i}|| \zeta\left|; i=1, \ldots, n, \psi_{m}(\zeta)=\lambda /\right| \zeta \mid \text {. }
$$

It is sufficient to estimate each term of right hand side.

$$
\begin{aligned}
\partial_{\psi}^{v} F_{(\alpha)}(x, \psi(\zeta)) \partial_{\xi}^{\tau}\left[\left(\partial_{\xi} \psi\right)^{\mu}\right] \\
\quad=\partial_{\psi}^{\tilde{\psi}} \partial_{\psi_{m}^{v}}^{v_{m}^{m}} F_{(\alpha)}(x, \psi(\zeta)) \partial_{\xi}^{\tau}\left[\left(\partial_{\xi} \tilde{\psi}\right)^{\tilde{\mu}}\left(\partial_{\xi} \psi_{m}\right)^{\mu_{m}}\right],
\end{aligned}
$$

where $\tilde{v}=\left(v_{i}\right)_{i=1, \ldots, n}, \tilde{\psi}=\left(\psi_{i}\right)_{i=1, \ldots, n}$,

$$
\tilde{\mu}=\left(\mu_{i j}\right)_{i, j=1, \ldots, n}, \mu_{m}=\left(\mu_{m j}\right)_{j=1, \ldots, n} .
$$

We compute these.

$$
\begin{aligned}
& \left(\partial_{\xi} \tilde{\psi}\right)^{\tilde{\mu}}=|\zeta|^{-|\underline{\underline{\tilde{\mu}}}|} \varphi_{1}(\zeta) \\
& \left(\partial_{\xi} \psi_{m}\right)^{\mu_{m}}=\lambda\left|\mu_{m}\right||\zeta|^{-2\left|\mu_{m}\right|} \varphi_{2}(\zeta) \\
& \left(\partial_{\xi} \psi\right)^{\mu}=\lambda\left|\mu_{m}\right||\zeta|^{-\left(|\overline{\underline{\underline{\mu}}}|+2\left|\mu_{m}\right|\right)} \varphi_{3}(\zeta) \\
& \partial_{\xi}^{\tau}\left[\left(\partial_{\xi} \psi\right)^{\mu}\right]=\lambda\left|\mu_{m}\right||\zeta|^{-\left(|\underline{\underline{\tilde{\mu}}}|+2\left|\mu_{m}\right|+|\tau|\right)} \varphi_{4}(\zeta),
\end{aligned}
$$

where $\varphi_{i}(\zeta)_{i=1,2,3,4}$ are homogeneous functions of order 0 in $\zeta$. On the other hand from the assumption for $F(x, \zeta)$ on $X \Xi \Lambda$ we obtain the followings.

$$
\left\|\partial_{\psi}^{v} F_{(\alpha)}(x, \zeta)\right\| \leqq c_{\alpha v}(|\zeta| / \lambda)^{\left(\delta|\alpha|+\varepsilon|\tilde{v}|+v_{m}+1\right)}
$$

Combining (2.14) and (2.15), we obtain that 


$$
\begin{aligned}
& \left\|\partial_{\psi}^{v} F_{(\alpha)}(x, \psi(\zeta)) \partial_{\xi}^{\tau}\left[\left(\partial_{\xi} \psi\right)^{\mu}\right]\right\| \leqq c \lambda^{-\kappa_{1}}|\zeta|^{\kappa_{2}}, \\
& \kappa_{1}=\left(\delta|\alpha|+\varepsilon|\tilde{v}|+v_{m}+1\right)-\left|\mu_{m}\right| \\
& \kappa_{2}=\left(\delta|\alpha|+\varepsilon|\tilde{v}|+v_{m}+1\right)-\left(|\underline{\tilde{\mu}}|+2\left|\mu_{m}\right|+|\tau|\right) .
\end{aligned}
$$

Since $\left|\mu_{m}\right|=v_{m},|\tilde{v}|=|\underline{\tilde{\mu}}|$ and $|\tilde{v}|+v_{m}+|\tau|=|\beta|$, so

$$
\begin{aligned}
& \kappa_{1}=\delta|\alpha|+\varepsilon|\beta|+1-\varepsilon v_{m}-\varepsilon|\tau|, \\
& \kappa_{2}=\delta|\alpha|+(\varepsilon-1)|\beta|+1-\varepsilon v_{m}-\varepsilon|\tau| .
\end{aligned}
$$

Considering that $\lambda /|\zeta| \leqq 1$, we obtain that

$$
\lambda^{-\kappa_{1}}|\zeta|^{\kappa_{2}} \leqq \lambda^{-(\delta|\alpha|+\varepsilon|\beta|+1)}|\zeta|^{(\delta|\alpha|+(\varepsilon-1)|\beta|+1)} .
$$

Thus, we conclude that

$$
\begin{aligned}
& \left\|\partial_{\psi}^{v} F_{(\alpha)}(x, \psi(\zeta)) \partial_{\xi}^{\tau}\left[\left(\partial_{\xi} \psi\right)^{\mu}\right]\right\| \\
& \quad \leqq c \lambda^{-(\delta|\alpha|+\varepsilon|\beta|+1)}|\zeta|^{(\delta|\alpha|+(\varepsilon-1)|\beta|+1)} .
\end{aligned}
$$

Summing these in $(v, \tau)$ up, we obtain the conclusion of this lemma with other constants $c_{\alpha \beta}$ when $m=0$. When $m \neq 0$, we consider $|\zeta|^{-m} F(x$, $\zeta)$, which is a homogeneous function of order 0 and satisfies the same estimates on $X \Xi \Lambda$ as $F(x, \zeta) .|\zeta|^{-m} F(x, \zeta)$ satisfies the estimates of the conclusion of this lemma by the previous discussion. Since $F(x, \zeta)$ $=|\zeta|^{m}\left\{|\zeta|^{-m} F(x, \zeta)\right\}$, we obtain the conclusion by using the Leibniz formula.

End of proof.

Lemma 2.6. In $(x, \xi, \lambda) \in R^{2 n+1} \cap\{\lambda>0\}$, for all $\alpha, \beta$ and $\gamma$ and for some constants $c_{\alpha \beta}$ and $c_{\alpha \beta \gamma}$ which do not depend on $\left.\left.(x, \xi, \lambda), 1\right) \sim 6\right)$ and 7) hold where $\zeta=(\xi, \lambda)$.

1) $\left\|\partial_{x}^{\alpha} \partial_{\xi}^{\beta} M(x, \xi, \lambda)\right\| \leqq c_{\alpha \beta}|\zeta|^{-|\beta|+1}$

2) $\left\|\partial_{x}^{\alpha} \partial_{\xi}^{\beta} B(x, \xi, \lambda)\right\| \leqq c_{\alpha \beta}|\zeta|^{-|\beta|}$

3) $\left\|\partial_{x}^{\alpha} \partial_{\xi}^{\beta} P_{-}(x, \xi, \lambda)\right\| \leqq c_{\alpha \beta}|\zeta|^{-|\beta|}$

4) $\left\|\partial_{x}^{\alpha} \partial_{\xi}^{\beta} D(x, \xi, \lambda)\right\| \leqq c_{\alpha \beta}|\zeta|^{-|\beta|}$ 
5) $\left\|\partial_{x}^{\alpha} \partial_{\xi}^{\beta} E(x, \xi, \lambda)\right\| \leqq c_{\alpha \beta} \lambda^{-(|\alpha|+|\beta|+2) / 2}|\zeta|^{(|\alpha|-|\beta|+2) / 2}$

6) $\left\|\partial_{x}^{\alpha} \partial_{\xi}^{\beta} J_{\gamma}^{1}(x, \xi, \lambda)\right\| \leqq c_{\alpha \beta \gamma} \lambda-(|\alpha|+|\beta|+2) / 2|\zeta|^{(|\alpha|-|\beta|) / 2}$ when $|\gamma|=1$.

$\left\|\partial_{x}^{\alpha} \partial_{\xi}^{\beta} J_{\gamma}^{1}(x, \xi, \lambda)\right\| \leqq c_{\alpha \beta \gamma} \lambda^{-(|\alpha|+|\beta|+|\gamma|+2) / 2}|\zeta|^{(|\alpha|-|\beta|-|\gamma|+2) / 2}$ when $|\gamma| \geqq 2$.

7) $\left\|\partial_{x}^{\alpha} \partial_{\xi}^{\beta} J_{\gamma}^{2}(x, \xi, \lambda)\right\| \leqq c_{\alpha \beta \gamma} \lambda^{-(|\alpha|+|\beta|+2) / 2}|\zeta|^{(|\alpha|-|\beta|) / 2}$ when $|\gamma|=1$.

$\left\|\partial_{x}^{\alpha} \partial_{\xi}^{\beta} J_{\gamma}^{2}(x, \xi, \lambda)\right\| \leqq c_{\alpha \beta \gamma} \lambda^{-(|\alpha|+|\beta|+|\gamma|+2) / 2}|\zeta|^{(|\alpha|-|\beta|-|\gamma|+2) / 2}$ when $|\gamma| \geqq 2$.

Proof. $E$ is homogeneous order 0 and satisfies 3) of Lemma 2.2 and $J_{\gamma}^{i}$ are homogeneous order $-|\gamma|$ and satisfy Lemma 2.4. We can apply Lemma 2.5 to these facts to obtain this lemma. End of proof.

Lemma 2.7. For all multi-indices $\alpha, \beta$ and $\gamma$ and for some positive constants $c_{\alpha \beta}, c_{\alpha \beta \gamma}$ and $a$,

1) $\left\|\partial_{x}^{\alpha} \partial_{\xi}^{\beta}\left\{(i \eta+M(x, \xi, \lambda))^{-1}\right\}\right\| \leqq c_{\alpha \beta}\left(|\eta|^{2}+|\zeta|^{2}\right)^{-(|\beta|+1) / 2}$

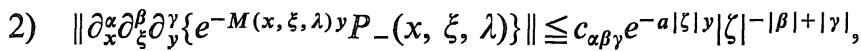
where $e^{-M(x, \xi, \lambda) y} P_{-}(x, \xi, \lambda) \equiv(2 \pi i)^{-1} \int_{\Gamma_{-}}(\mu+M(x, \xi, \lambda))^{-1} e^{+\mu y} d \mu$.

Proof. 1) follows from the fact that $i \eta+M(x, \xi, \lambda)$ is non-singular for $(\eta, \xi, \lambda) \neq 0$ and does not depend on $x$ outside a ball in $x$-space. Since $\Gamma_{-}$is taken not to depend on $(x, \xi, \lambda)$ if $|\zeta|^{2} \equiv|\xi|^{2}+|\lambda|^{2}=1$, we obtain 2) using 1) and homogeneity of $M(x, \xi, \lambda)$ in $(\xi, \lambda)$.

End of proof.

\section{§3. Results from Theory of Pseudo-Differential Operators}

In this section we shall mention the results on pseudo-differential operators obtained by A.P. Calderon and $\mathbb{R}$. Vaillancourt [4] and $\mathbb{L}$. Hörmander [8]. We shall apply these to some pseudo-differential operators which we need in this paper. In order to apply these to our proof we have to make it clear how norms of pseudo-differential operators depend on parameters. So we shall give rough proves again though they are almostly same as ones by $\mathbb{L}$. Hörmander. 
We consider pseudo-differential operators on the space of functions (distributions) valued in a Hilbert space $X$, that is, symbols of pseudodifferential operators are $C^{\infty}$-functions valued in $B L(X)$ the space of bounded linear operators on $X$ with some additional conditions. Let $C^{\infty}\left(R^{s}, X\right)$ and $C^{\infty}\left(R^{s}, B L(X)\right)$ stand for the space of $X$ and $B L(X)$ valued $C^{\infty}$-functions on $R^{s}$ with respect to the topologies by the norms of $X$ and $B L(X)$, respectively. We denote the norms of $X$ and $B L(X)$ by $|\cdot|$ and the norms of $H^{m}\left(R^{s}, X\right)$ the Sobolev spaces of $X$-valued distribution by $\|\cdot\|_{m}$.

Lemma 3.1. Let $p(x, \xi) \in C^{\infty}\left(R^{n} \times R^{n}, B L(X)\right)$ and assume that with $0 \leqq \delta \leqq \rho \leqq 1(\delta \neq 1)$

$$
\left|p_{(\beta)}^{(\alpha)}(x, \xi)\right| \leqq c_{\alpha \beta}(1+|\xi|)^{-\rho|\alpha|+\delta|\beta|}
$$

for $(x, \xi) \in R^{n} \times R^{n}$. Then there is a constant $c$, depending on the constants $c_{\alpha \beta}$, such that

$$
\|p(x, \partial) u\|_{0} \leqq c\|u\|_{0}, \quad u \in C_{0}^{\infty}\left(R^{n}, X\right)
$$

where $p_{(\beta)}^{(\alpha)}(x, \xi) \equiv \partial_{\xi}^{\alpha} \partial_{x}^{\beta} P(x, \xi)$. (Refer to Hörmander [8] and Calderon and Vaillancourt [4] for proof. There is nothing that should be changed in them though symbols take their values in bounded operators on a Hilbert space.)

Lemma 3.2. Let $p(x, \xi)$ and $q(x, \xi) \in C^{\infty}\left(R^{n} \times R^{n}, B L(X)\right)$ and assume that $p(x, \xi)$ and $q(x, \xi)$ vanish for $x$ outside a ball $\{x ;|x| \leqq R\}$ and that for all multi-indices $\alpha, \beta$ we have constants $c_{|\alpha||\beta|}, m_{i}, \delta_{i}$ and $\rho_{i}$, where $i=1$ or 2 , such that

$$
\begin{aligned}
& \left|p_{(\beta)}^{(\alpha)}(x, \xi)\right| \leqq c_{|\alpha||\beta|}(1+|\xi|)^{m_{1}+\delta_{1}|\beta|-\rho_{1}|\alpha|} \\
& \left|q_{(\beta)}^{(\alpha)}(x, \xi)\right| \leqq c_{|\alpha||\beta|}(1+|\xi|)^{m_{2}+\delta_{2}|\beta|-\rho_{2}|\alpha|}
\end{aligned}
$$

$0 \leqq \delta_{1}<\rho_{2} \leqq 1$.

Then, for any integer $m$ we have

$$
\begin{aligned}
& \left\|q(x, \partial) p(x, \partial) u(x)-\sum_{|\alpha|<N}(i)^{|\alpha|}(\alpha !)^{-1} q^{(\alpha)}(x, \partial) \circ p_{(\alpha)}(x, \partial) u(x)\right\| \\
& \quad \leqq b(N, L, n) R^{2 n} c_{\max }(N, L) \cdot\|u(x)\|_{m}, \quad \text { for } \quad u(x) \in C_{0}^{\infty}\left(R^{n}, X\right)
\end{aligned}
$$


where $b(N, L, n)$ are constants which depend only on $N, L$ and $n$, $c_{\max }(N, L)=\max _{-1 \leqq l-n \leqq 1}\left[c_{N l}\right] \cdot \max _{-1 \leqq l-n-N \leqq 1+L}\left[c_{0 l}\right]$

and

$$
\left\{\begin{array}{l}
m_{1}+m_{2}+\left(\delta_{1}+\delta_{2}\right)(n+1)+\left(\delta_{1}-\rho_{2}\right) N \leqq m \\
m_{1}+\delta_{1}(n+1)+\delta_{1} N+\left(\delta_{1}-1\right) L \leqq m \\
m_{2}+\delta_{2}(n+1)-\rho_{2} N \leqq 0
\end{array}\right.
$$

Proof. We consider a partial sum of the Taylor expansion of $q(x, \eta)$ in $\eta$ at $\xi$.

$$
\begin{aligned}
q(x, \eta)= & \sum_{|\alpha|<N} q^{(\alpha)}(x, \xi)(i)^{|\alpha|}(\alpha !)^{-1}(\eta-\xi)^{\alpha} \\
& +\sum_{|\alpha|=N} r^{(\alpha)}(x, \xi, \eta-\xi)(i)^{N}(\alpha !)^{-1}(\eta-\xi)^{\alpha} \\
r^{(\alpha)}(x, \xi, \eta-\xi)=|\alpha| & \int_{0}^{1} q^{(\alpha)}(x, \xi+\theta(\eta-\xi))(1-\theta)^{|\alpha|-1} d \theta
\end{aligned}
$$

Now we obtain the following Lemma 3.3 for $\hat{r}^{(\alpha)}(\zeta, \xi, \eta-\xi)$ which is the Fourier transform of $r^{(\alpha)}(x, \xi, \eta-\xi)$ in $x$.

\section{Lemma 3.3.}

$$
\begin{aligned}
& \left|\hat{r}^{(\alpha)}(\zeta, \xi, \eta-\xi)\right| \\
& \quad \leqq\left\{\sup _{0 \leqq \theta \leqq 1}(1+|\xi+\theta(\eta-\xi)|)^{m_{2}+\delta_{2}(L+n-1)-\rho_{2}|\alpha|}\right\} \\
& \quad \times\left\{\max _{-1 \leqq l-n \leqq L-1}\left[c_{|\alpha| l}\right]\right\} 2^{L} S_{n} R^{n}(1+|\zeta|)^{-L}|\zeta|^{1-n}
\end{aligned}
$$

On the other hand we have Lemma 3.4 for $\hat{p}_{(\alpha)}(\eta, \xi)$.

\section{Lemma 3.4.}

$$
\begin{aligned}
& (1+|\eta|)^{L}|\eta|^{n-1}\left|\hat{p}_{(\alpha)}(\eta, \xi)\right| \\
& \quad \leqq 2^{L} S_{n} R^{n} \cdot \max _{0 \leqq l-|\alpha|-n+1 \leqq L}\left[c_{0 l}\right] \cdot(1+|\xi|)^{m_{1}+\delta_{1}(|\alpha|+L+n-1)}
\end{aligned}
$$

We now put $v(x) \equiv q(x, \partial) p(x, \partial) u(x)$. Then we have 


$$
\begin{aligned}
\hat{v}(\zeta)= & \iint \hat{q}(\zeta-\eta, \eta) \hat{p}(\eta-\xi, \xi) \hat{u}(\xi) d \xi d \eta \\
= & \sum_{|\alpha|<N}(i)^{|\alpha|}(\alpha !)^{-1} \iint \hat{q}^{(\alpha)}(\zeta-\eta, \xi) \hat{p}_{(\alpha)}(\eta-\xi, \xi) \hat{u}(\xi) d \xi d \eta \\
& +\sum_{|\alpha|=N}(i)^{|\alpha|}(\alpha !)^{-1} \iint \hat{r}^{(\alpha)}(\zeta-\eta, \xi, \eta-\xi) \hat{p}_{(\alpha)}(\eta-\xi, \xi) \hat{u}(\xi) d \xi d \eta \\
= & \sum_{|\alpha|<N}(i)^{|\alpha|}(\alpha !)^{-1} \int \widehat{q^{(\alpha)} \cdot p_{(\alpha)}(\zeta-\xi, \xi) \hat{u}(\xi) d \xi} \\
& +\sum_{|\alpha|=N}(i)^{|\alpha|}(\alpha !)^{-1} \iint \hat{r}^{(\alpha)}(\zeta-\eta, \xi, \eta-\xi) \hat{p}_{(\alpha)}(\eta-\xi, \xi) \hat{u}(\xi) d \xi d \eta
\end{aligned}
$$

where $\left.\widehat{q^{(\alpha)} \cdot p_{(\alpha)}(\zeta}, \xi\right)=\int e^{-i x \zeta} q^{(\alpha)}(x, \xi) p_{(\alpha)}(x, \xi) d x$. This equality shows that it suffices to estimate the last terms

$$
\sum_{|\alpha|=N}(i)^{N}(\alpha !)^{-1} \iint \hat{r}^{(\alpha)}(\zeta-\eta, \xi, \eta-\xi) \hat{p}_{(\alpha)}(\eta-\xi, \xi) \hat{u}(\xi) d \xi d \eta
$$

in the form of Lemma 3.2.

\section{Lemma 3.5.}

$$
\begin{aligned}
& \left|\hat{r}^{(\alpha)}(\zeta-\eta, \xi, \eta-\xi) \hat{p}_{(\alpha)}(\eta-\xi, \xi)\right| \\
& \quad \leqq 2^{4+\max \left(2 L, \rho_{2} N-m_{2}-\delta_{2}(n+1)\right)}\left(S_{n} R^{n}\right)^{2} \\
& \quad \times \max _{-1 \leqq l-n \leqq 1}\left[c_{N}\right] \cdot \max _{-1 \leqq l-N-n \leqq L+1}\left[c_{0 l}\right] \\
& \quad \times(1+|\zeta-\eta|)^{-2}|\zeta-\eta|^{1-n}(1+|\eta-\xi|)^{-2}|\eta-\xi|^{1-n}(1+|\xi|)^{m}
\end{aligned}
$$

where $\quad m_{1}+m_{2}+\left(\delta_{1}+\delta_{2}\right)(n+1)+\left(\delta_{1}-\rho_{2}\right) N \leqq m$

$$
\begin{aligned}
& m_{1}+\delta_{1}(n+1)+\delta_{1} N+\left(\delta_{1}-1\right) L \leqq m \\
& m_{2}+\delta_{2}(n+1)-\rho_{2} N \leqq 0
\end{aligned}
$$

If we prove this lemma for $\hat{r}^{(\alpha)}(\zeta-\eta, \xi, \eta-\xi) \hat{p}_{(\alpha)}(\eta-\xi$, $\xi$, we obtain the followings by Hausdorff-Young inequality.

$$
\left\|v(x)-\sum_{|\alpha|<N}(i)^{|\alpha|}(\alpha !)^{-1} q^{(\alpha)}(x, \partial) \circ p_{(\alpha)}(x, \partial) u(x)\right\|
$$




$$
\begin{aligned}
& \quad=\left\|\sum_{|\alpha|=N}(i)^{N}(\alpha !)^{-1} \iint \hat{r}^{(\alpha)}(\zeta-\eta, \xi, \eta-\xi) \hat{p}_{(\alpha)}(\eta-\xi, \xi) \hat{u}(\xi) d \xi d \eta\right\| \\
& \quad \leqq b(N, L, n) R^{2 n} c_{\max }(N, L)\|u(x)\|_{m} ; \\
& b(N, L, n) \\
& =2^{4+\max \left(2 L, \rho_{2} N-m_{2}-\delta_{2}(n+1)\right)} S_{n}^{2}\left(\sum_{|\alpha|=N} 1 / \alpha !\right)\left(\int(1+|\zeta|)^{-2}|\alpha|^{1-n} d \zeta\right)^{2} .
\end{aligned}
$$

The proof of Lemma 3.2 is complete if we verify Lemmas $3.3 \sim 5$.

End of proof of Lemma 3.2.

It is easy to verify Lemmas 3.3 and 3.4 using the assumptions for $p(x, \xi)$ and $q(x, \xi)$. So, we prove Lemma 3.5 from Lemmas 3.3 and 3.4 .

(Proof of Lemma 3.5) When $|\eta-\xi| \leqq|\xi| / 2$, we obtain (3.1) from Lemma 3.3 and (3.2) from Lemma 3.4 because $1+|\xi+\theta(\eta-\xi)| \geqq(1+|\xi|) / 2$. Combining (3.1) and (3.2), we conclude Lemma 3.5 if $|\eta-\xi| \leqq|\xi| / 2$.

$$
\begin{aligned}
& \left|\hat{r}^{(\alpha)}(\zeta-\eta, \xi, \eta-\xi)\right|
\end{aligned}
$$

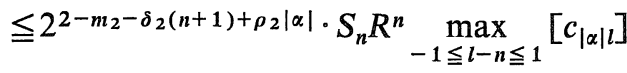

$$
\begin{aligned}
& \times(1+|\zeta-\eta|)^{-2}|\zeta-\eta|^{1-n}(1+|\zeta|)^{m_{2}+\delta_{2}(n+1)-\rho_{2}|\alpha|},
\end{aligned}
$$

where $m_{2}+\delta_{2}(n+1)-\rho_{2}|\alpha| \leqq 0$.

$$
\begin{aligned}
& \left|\hat{p}_{(\alpha)}(\eta-\xi, \xi)\right| \\
& \quad \leqq 2^{2} S_{n} R^{n} \max _{-1 \leqq l-n-|\alpha| \leqq 1}\left[c_{0 l}\right] \\
& \quad \times(1+|\eta-\xi|)^{-2}|\eta-\xi|^{1-n}(1+|\xi|)^{m_{1}+\delta_{1}(|\alpha|+n+1)} .
\end{aligned}
$$

When $|\eta-\xi| \geqq|\xi| / 2$, we obtain (3.3) from Lemma 3.3 and (3.4) from Lemma 3.4 because $1+|\eta-\xi| \geqq(1+|\xi|) / 2$. Combining (3.3) and (3.4), again, we complete the proof of Lemma 3.5 .

$$
\begin{aligned}
& \left|\hat{r}^{(\alpha)}(\zeta-\eta, \xi, \eta-\xi)\right| \\
& \quad \leqq 2^{2} S_{n} R^{n} \max _{-1 \leqq l-n \leqq 1}\left[c_{|\alpha| l}\right](1+|\zeta-\eta|)^{-2}|\zeta-\eta|^{1-n}
\end{aligned}
$$


where $m_{2}+\delta_{2}(n+1)-\rho_{2}|\alpha| \leqq 0$.

$$
\begin{aligned}
& \left|\hat{p}_{(\alpha)}(\eta-\xi, \xi)\right| \\
& \quad \leqq 2^{2+2 L} S_{n} R_{-1 \leqq l-|\alpha|-n \leqq L+1}^{n}\left[c_{0 l}\right] \\
& \quad \times(1+|\eta-\xi|)^{-2}|\eta-\xi|^{1-n}(1+|\xi|)^{m_{1}+\delta_{1}(|\alpha|+L+n+1)-L}
\end{aligned}
$$

End of proof.

Lemma 3.6. Let $q(\xi)$ be constant in $x$. If $p(x, \xi)$ and $q(\xi)$ satisfy the hypotheses of Lemma 3.2 except for the hypothesis that $q(\xi)$ vanishes in $x$ outside a ball, then the result of Lemma 3.2 is valid with another constant $b^{\prime}(N, L, n) R^{n} c^{\prime}{ }_{\text {max }}(N, L)$ such that $c^{\prime}(N, L) \equiv \max _{-1 \leqq l-N-n \leqq L+1}\left[c_{0 l}\right] c_{N l}$.

Proof. From constantness of $q(\xi)$ in $x$ we have that

$$
\begin{aligned}
\hat{v}(\zeta)= & \sum_{|\alpha|<N}(i)^{|\alpha|}(\alpha !)^{-1} \widehat{q^{(\alpha)} p_{(\alpha)}}(\zeta-\xi, \xi) \hat{u}(\xi) d \xi \\
& +\sum_{|\alpha|=N}(i)^{|\alpha|}(\alpha !)^{-1} \int r^{(\alpha)}(\xi, \zeta-\xi) \hat{p}_{(\alpha)}(\zeta-\xi, \xi) \hat{u}(\xi) d \xi .
\end{aligned}
$$

We have further

$$
\begin{aligned}
& \left|r^{(\alpha)}(\xi, \eta-\xi)\right| \leqq \sup _{0 \leqq \theta \leqq 1}(1+|\xi+\theta(\eta-\xi)|)^{m_{2}-\rho_{2}|\alpha| \cdot c_{|\alpha| 0}} \\
& \quad \leqq\left\{\begin{array}{lll}
2^{\rho_{2}|\alpha|-m_{2}}(1+|\xi|)^{m_{2}-\rho_{2}|\alpha|} c_{|\alpha| 0} & \text { if } & |\eta-\xi| \leqq|\xi| / 2 \\
c_{|\alpha| 0} & \text { if } & |\eta-\xi| \geqq|\xi| / 2,
\end{array}\right.
\end{aligned}
$$

where $m_{2}-\rho_{2}|\alpha| \leqq 0$.

Combining (3.2), (3.4) and (3.5) we can estimate as

$$
\begin{aligned}
& \left\|\hat{v}(\zeta)-\sum_{|\alpha|<N}(i)^{|\alpha|}(\alpha !)^{-1} \int q^{(\alpha)} p_{(\alpha)}(\zeta-\xi, \xi) \hat{u}(\xi) d \xi\right\| \\
& \quad \leqq b^{\prime}(N, L, n) R^{n} c^{\prime}{ }_{\max }(N, L)\left\|(1+|\xi|)^{m} \hat{u}(\xi)\right\| \\
& \quad b^{\prime}(N, L, n) \equiv 2^{2+\max \left(2 L, \rho_{2} N-m_{2}\right)}\left(\sum_{|\alpha|=N} 1 / \alpha !\right) \int(1+|\zeta|)^{-2}|\zeta|^{1-n} d \zeta
\end{aligned}
$$

if $m_{1}+m_{2}+\delta_{1}(n+1)+\left(\delta_{1}-\rho_{2}\right) N \leqq m, \quad m_{1}+\delta_{1}(n+1)+\delta_{1} N+\left(\delta_{1}-1\right) L \leqq m$ 
and $m_{2}-\rho_{2} N \leqq 0$.

End of proof.

Lemma 3.7. Let $p(x, \xi, \lambda)$ and $q(x, \xi, \lambda) \in C^{\infty}\left(R^{n} \times R^{n}, B L(X)\right)$ with a parameter $\lambda \in(0, \infty)$ and assume that $p(x, \xi, \lambda)$ and $q(x, \xi, \lambda)$ are constant in $x$ outside $a$ ball $\{x ;|x| \leqq R\}$ and that for all multi-indices $\alpha, \beta$ we have constants $c_{|\alpha||\beta|}, m_{i}, \delta_{i}$ and $\rho_{i}$, where $i=1$ or 2 , such that

$$
\begin{aligned}
& \left|p_{(\beta)}^{(\alpha)}(x, \xi, \lambda)\right| \leqq c_{|\alpha||\beta|} \lambda-|\alpha| \cdot(|\zeta| / \lambda)^{m_{1}+\delta_{1}|\beta|-\rho_{1}|\alpha|} \\
& \left|q_{(\beta)}^{(\alpha)}(x, \xi, \lambda)\right| \leqq c_{|\alpha||\beta|} \lambda^{-|\alpha|}(|\zeta| / \lambda)^{m_{2}+\delta_{2}|\beta|-\rho_{2}|\alpha|},
\end{aligned}
$$

$0 \leqq \delta_{1}<\rho_{2} \leqq 1$ and $\zeta=(\xi, \lambda)$.

Then for any integer $m$ we have

$$
\begin{aligned}
& \left\|q(x, \partial, \lambda) p(x, \partial, \lambda) u(x)-\sum_{|\alpha|<N}(i)^{|\alpha|}(\alpha !)^{-1} q^{(\alpha)}(x, \partial, \lambda) \circ p_{(\alpha)}(x, \partial, \lambda) u(x)\right\| \\
& \quad \leqq c \lambda^{-N-m+2}\left\|\Lambda^{m}(\lambda) u\right\|, \text { for } u(x) \in C_{0}^{\infty}\left(R^{n}, X\right)
\end{aligned}
$$

where $c$ is a constant which depends only on $N, L, n, R$ and $c_{|\alpha||\beta|}$ and $m_{i}, \delta_{i}, \rho_{i}, N, L$ and $m$ satisfy the relations in Lemma 3.2.

Remark. Many times we use pseudo-differential operators $\Lambda^{s}(\lambda)$ for all real number $s$ whose symbols are $\left(|\xi|^{2}+\lambda^{2}\right)^{s / 2}$.

Proof. Let $u \in C_{0}^{\infty}\left(R^{n}, X\right), v \equiv q p u-\sum_{|\alpha|<N}(i)^{|\alpha|} / \alpha ! \cdot q^{(\alpha)_{\circ}} p_{(\alpha)} u, \tilde{p}(x, \xi, \lambda) \equiv$ $p(x / \lambda, \xi \lambda, \lambda), \tilde{q}(x, \xi, \lambda) \equiv q(x / \lambda, \xi \lambda, \lambda), u_{1}(x) \equiv u(x / \lambda) \quad$ and $\quad v_{1}(x)=v(x / \lambda)$.

Then

$$
v_{1}(x)=\tilde{q} \tilde{p} u_{1}-\sum_{|\alpha|<N}(i)^{|\alpha|} / \alpha ! \cdot \tilde{q}^{(\alpha)} \circ \tilde{p}_{(\alpha)} u_{1}
$$

Functions $\tilde{p}$ and $\tilde{q}$ satisfy the hypotheses of Lemma 3.2 and 3.6 as followings

$$
\begin{aligned}
& \left|\tilde{p}_{(\beta)}^{(\alpha)}(x, \xi, \lambda)\right| \leqq c_{|\alpha||\beta|} \lambda^{-|\beta|}(1+|\xi|)^{\left(m_{1}+\delta_{1}|\beta|-\rho_{1}|\alpha|\right)} \\
& \left|\tilde{q}_{(\beta)}^{(\alpha)}(x, \xi, \lambda)\right| \leqq c_{|\alpha||\beta|} \lambda^{-|\beta|}(1+|\xi|)^{\left(m_{2}+\delta_{2}|\beta|-\rho_{2}|\alpha|\right)} .
\end{aligned}
$$

So we apply Lemma 3.2 and Lemma 3.6 to (3.6). $R^{n}, c_{\max }(N, L)$ in Lemma 3.2 and $c_{\text {max }}^{\prime}(N, L)$ in Lemma 3.6 are less than $c \lambda^{n}, c \lambda^{-N-2 n+2}$ 
and $c \lambda^{-N-n+1}$ for some constant $c$, respectively. Thus,

$$
\left\|v_{1}\right\| \leqq c \lambda^{-N+2}\left\|\Lambda^{m}(1) u_{1}\right\|
$$

Changing variables, we obtain the estimate of this lemma.

End of proof.

Lemma 3.8. Let $p(x, \xi, \lambda)$ satisfy the hypotheses of Lemma 3.7, where $0 \leqq \delta_{1} \leqq \rho_{1} \leqq 1$ and $\delta_{1} \neq 1$. Then we obtain

$$
\begin{aligned}
\left\|\Lambda^{m}(\lambda) p(x, \partial, \lambda) u\right\| \leqq & c_{0} \lambda^{-m_{1}}\left\|\Lambda^{m+m_{1}}(\lambda) u\right\| \\
& +c_{m} \lambda^{-m_{1}-\delta_{1}}\left\|\Lambda^{m+m_{1}+\delta_{1}-1}(\lambda) u\right\|
\end{aligned}
$$

especially

$$
\left\|\Lambda^{m}(\lambda) p(x, \partial, \lambda) u\right\| \leqq c_{m} \lambda^{-m_{1}}\left\|\Lambda^{m+m_{1}}(\lambda) u\right\|
$$

for $\lambda \in[1, \infty)$ and for any real number $m$, where $c_{0}$ does not depend on $\mathrm{m}$.

Proof. When $m=0$, we prove the estimate in the same way as in Lemma 3.7 using Lemma 3.1 instead of Lemma 3.2. $\tilde{p}(x, \xi, \lambda)$ $\equiv p(x / \lambda, \xi \lambda, \lambda) \quad$ satisfies the hypotheses of Lemma 3.1 with constants $c_{|\alpha||\beta|}$ which do not depend on $\lambda$ if $\lambda \geqq 1$. If we put $v=p(x, \partial, \lambda) u$, $u_{1}(x) \equiv u(x / \lambda) \quad$ and $\quad v_{1}(x) \equiv v(x / \lambda)$, then $v_{1}=\tilde{p}(x, \partial, \lambda) u_{1} \quad$ and $\quad\left\|v_{1}\right\| \leqq$ $c\left\|\Lambda^{m_{1}}(1) u_{1}\right\|$ by Lemma 3.1, where $c$ does not depend on $\lambda \in[1, \infty)$. Changing variables, we obtain that

$$
\|v\| \leqq c \lambda^{-m_{1}}\left\|\Lambda^{m_{1}}(\lambda) u\right\|, \quad \lambda \in[1, \infty) .
$$

Let us put $q(x, \xi, \lambda) \equiv(|\zeta| / \lambda)^{m}$. Then $p(x, \xi, \lambda)$ and $q(x, \xi, \lambda)$ satisfy the hypotheses of Lemma 3.7, where $m_{2}=m, \delta_{2}=0$ and $\rho_{2}=1$. This means that it is enough to estimate the finite numbers of the pseudo-differential operators with symbols $r(\gamma, x, \xi, \lambda) \equiv q^{(\gamma)}(x, \xi, \lambda) p_{(\gamma)}(x, \xi, \lambda)$ because from Lemma 3.7 the error term $q p u-\sum_{|\alpha|<N}(i)^{|\alpha|} / \alpha ! \cdot q^{(\alpha)} \circ p_{(\alpha)} u$ is bounded by $c_{N} \lambda^{-m-m_{1}-1}\left\|\Lambda^{m+m_{1}-1}(\lambda) u\right\|$ if $N$ is sufficiently large. For some other constants $c_{|\alpha||\beta||\gamma|}$,

$$
\left|r_{(\beta)}^{(\alpha)}(\gamma, x, \ddot{\zeta}, \lambda)\right|
$$




$$
\leqq c_{|\alpha||\beta||\gamma|} \lambda^{-|\alpha|-|\gamma|}(|\zeta| / \lambda)^{m+m_{1}+\delta_{1}|\beta|-\rho_{1}|\alpha|+\left(\delta_{1}-1\right)|\gamma|} 。
$$

So we can use the result which we have already proved in the case $m=0$.

$$
\|r(\gamma, x, \partial, \lambda) u\| \leqq c_{\gamma} \lambda^{-m-m_{1}-\delta_{1}|\gamma|}\left\|\Lambda^{m+m_{1}+\left(\delta_{1}-1\right)|\gamma|}(\lambda) u\right\|
$$

Summing these and the error term up, we obtain that

$$
\begin{aligned}
& \lambda^{-m}\left\|\Lambda^{m}(\lambda) p(x, \partial, \lambda) u\right\|=\|q(x, \partial, \lambda) p(x, \partial, \lambda) u\| \\
& \leqq \\
& c_{0} \lambda^{-m-m_{1}}\left\|\Lambda^{m+m_{1}}(\lambda) u\right\|+\sum_{1 \leqq|\gamma|<N} \lambda^{-m-m_{1}-\delta_{1}|\gamma|}\left\|\Lambda^{m+m_{1}+\left(\delta_{1}-1\right)|\gamma|}(\lambda) u\right\| \\
& \quad+c_{N} \lambda^{-m-m_{1}-1}\left\|\Lambda^{m+m_{1}-1}(\lambda) u\right\|, \quad \lambda \in[1, \infty) \\
& \leqq \\
& c_{0} \lambda^{-m-m_{1}}\left\|\Lambda^{m+m_{1}}(\lambda) u\right\|+c_{m} \lambda^{-m-m_{1}-\delta_{1}}\left\|\Lambda^{m+m_{1}+\delta_{1}-1}(\lambda) u\right\|
\end{aligned}
$$

because $\quad \lambda^{\left(1-\delta_{1}\right) s}\left\|\Lambda^{\left(\delta_{1}-1\right) s}(\lambda) u\right\| \leqq\|u\|$.

End of proof.

Lemma 3.9. Let $p(x, \xi, \lambda)$ and $q(x, \xi, \lambda)$ satisfy the hypotheses of Lemma 3.7, where $0 \leqq \delta_{1} \leqq \rho_{1} \leqq 1, \delta_{1} \neq 1$ and $0 \leqq \delta_{2}<1$. Then for any real number $s$ and integer $m$ and for sufficiently large $N$ 's we have

$$
\begin{aligned}
& \| \Lambda^{s}(\lambda)\{q(x, \partial, \lambda) \cdot p(x, \partial, \lambda) \\
&\left.-\sum_{|\alpha|<N}(i)^{|\alpha|} / \alpha ! \cdot q^{(\alpha)}(x, \partial, \lambda) \circ p_{(\alpha)}(x, \partial, \lambda)\right\} u \| \\
& \leqq c \lambda^{-N+s-m+2}\left\|\Lambda^{m}(\lambda) u\right\|, \quad \text { for } \quad u \in C_{0}^{\infty}\left(R^{n}, X\right) .
\end{aligned}
$$

Proof. It is trivial if $p$ is constant in $x$. So we may assume that the support of $p$ in $x$ is bounded. Let us put $r(x, \partial, \lambda)=\Lambda^{s}(\lambda) \lambda^{-s}$. Since $q$ and $r, p$ and $\left.\lambda^{|\alpha|}\right|^{(\alpha)} \circ q_{(\alpha)}$, and, $\lambda^{|\beta|} q^{(\beta)} \circ p_{(\beta)}$ and $r$ satisfy the hypotheses of Lemma 3.7, we have (3.7), (3.8) and (3.9). We have also (3.10) by combining (3.7) and (3.8), and the result of this lemma by combining (3.9) and (3.10) for sufficiently large $N$ 's.

$$
\begin{aligned}
& \left\|\left\{r \cdot q-\sum_{|\alpha|<N}(i)^{|\alpha|} / \alpha ! \cdot r^{(\alpha)} \circ q_{(\alpha)}\right\} p u\right\| \\
& \leqq c \lambda^{-N-m+m_{1}+2}\left\|\Lambda^{m-m_{1}}(\lambda) p u\right\| \\
& \leqq c \lambda^{-N-m+2}\left\|\Lambda^{m}(\lambda) u\right\|
\end{aligned}
$$




$$
\begin{aligned}
& \left\|\left\{r^{(\alpha)} \circ q_{(\alpha)} \cdot p-\sum_{|\alpha+\beta+\gamma|<N}(i)^{|\beta+\gamma|} / \beta ! \gamma ! \cdot r^{(\alpha+\gamma)} \circ q_{(\alpha)}^{(\beta)} \circ p_{(\beta+\gamma)}\right\} u\right\| \\
& \leqq c \lambda^{-N-m+2}\left\|\Lambda^{m}(\lambda) u\right\|
\end{aligned}
$$

$$
\begin{aligned}
& \|\left\{r \cdot q^{(\beta)} \circ p_{(\beta)}-\sum_{|\alpha+\beta+\gamma|<N}(i)^{\left.|\alpha+\gamma| / \alpha ! \gamma ! \cdot r^{(\alpha+\gamma)} \circ q_{(\alpha)}^{(\beta)} \circ p_{(\beta+\gamma)}\right\} u \|}\right. \\
& \quad \leqq c \lambda^{-N-m+2}\left\|\Lambda^{m}(\lambda) u\right\|
\end{aligned}
$$

$$
\begin{aligned}
& \|\left\{r \cdot q \cdot p-\sum_{|\alpha+\beta+\gamma|<N}(i)^{\left.|\alpha+\beta+\gamma| / \alpha ! \beta ! \gamma ! \cdot r^{(\alpha+\gamma)} \circ q_{(\alpha)}^{(\beta)} \circ p_{(\beta+\gamma)}\right\} u \|}\right. \\
& \leqq c \lambda^{-N-m+2}\left\|\Lambda^{m}(\lambda) u\right\| \text {. }
\end{aligned}
$$

There are Lemmas 2.6 and 2.7 for $M, B, P_{-}, D, E, J_{\gamma}^{i}, e^{-M y_{\circ} P_{-}}$and $(i \eta+M)^{-1}$, which are defined in section 2 . So these are pseudo-differential operators to which Lemmas 3.7, 3.8 and 3.9 can be applied.

Lemma 3.10. Let $h$ be an element of $C_{0}^{\infty}\left(R^{n}\right)$ and $\lambda \in[1, \infty)$.

1) $\left\|\Lambda^{s}(\lambda) M\left(x, \partial_{x}, \lambda\right) h\right\| \leqq c\left\|\Lambda^{s+1}(\lambda) h\right\|$

2) $\left\|\Lambda^{s}(\lambda) B\left(x, \partial_{x}, \lambda\right) h\right\| \leqq c\left\|\Lambda^{s}(\lambda) h\right\|$

3) $\left\|\Lambda^{s}(\lambda) P_{-}\left(x, \partial_{x}, \lambda\right) h\right\| \leqq c\left\|\Lambda^{s}(\lambda) h\right\|$

4) $\left\|\Lambda^{s}(\lambda) D\left(x, \partial_{x}, \lambda\right) h\right\| \leqq c\left\|\Lambda^{s}(\lambda) h\right\|$

5) $\left\|\Lambda^{s}(\lambda) E\left(x, \partial_{x}, \lambda\right) h\right\| \leqq c \lambda^{-1}\left\|\Lambda^{s+1}(\lambda) h\right\|$

6) $\left\|\Lambda^{s}(\lambda) J_{\gamma}^{1}\left(x, \partial_{x}, \lambda\right) h\right\| \leqq c \lambda^{-1}\left\|\Lambda^{s}(\lambda) h\right\| \quad ;|\gamma|=1$

7) $\left\|\Lambda^{s}(\lambda) J_{\gamma}^{1}\left(x, \partial_{x}, \lambda\right) h\right\| \leqq c \lambda^{-|\gamma| / 2-1}\left\|\Lambda^{s-|\gamma| / 2+1}(\lambda) h\right\| ;|\gamma| \geqq 2$

8) $\left\|\Lambda^{s}(\lambda) J_{\gamma}^{2}\left(x, \partial_{x}, \lambda\right) h\right\| \leqq c \lambda^{-1}\left\|\Lambda^{s}(\lambda) h\right\| \quad ;|\gamma|=1$

9) $\left\|\Lambda^{s}(\lambda) J_{\gamma}^{2}\left(x, \partial_{x}, \lambda\right) h\right\| \leqq c \lambda^{-|\gamma| / 2-1}\left\|\Lambda^{s-|\gamma| / 2+1}(\lambda) h\right\| ;|\gamma| \geqq 2$

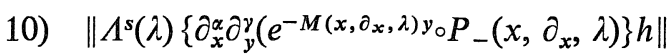

$$
\leqq c\left\{\int e^{\left.-2 a|\zeta| y|\zeta|^{2(s+|\gamma|)}|\hat{h}(\zeta)|^{2} d \xi\right\}^{1 / 2}}\right.
$$

$$
\leqq c e^{-a \lambda y}\left\|\Lambda^{s+|\gamma|}(\lambda) h\right\|
$$


11) $\left\{\int_{0}^{\infty} \| \Lambda^{s}(\lambda)\left\{\partial_{x}^{\alpha} \partial_{y}^{\gamma}\left(e^{\left.\left.-M\left(x, \partial_{x}, \lambda\right) y_{\circ} P_{-}\left(x, \partial_{x}, \lambda\right)\right\} h \|^{2} d y\right\}^{1 / 2}}\right.\right.\right.$

$$
\leqq c\left\|\Lambda^{s+|\gamma|-1 / 2}(\lambda) h\right\|
$$

12) $\left\|\left(i \eta-\Lambda^{1}(\lambda)\right)^{\gamma} \Lambda^{s-1}(\lambda)\left\{\partial_{x}^{\alpha}\left(A^{-\gamma}\left(x, \partial_{x}, \eta, \lambda\right)\right)\right\} h\right\|$

$$
\leqq c\left\|\Lambda^{s-1}(\lambda) h\right\|,
$$

where $A^{-\gamma}(x, \xi, \eta, \lambda) \equiv\{i \eta+M(x, \xi, \lambda)\}^{-\gamma}$

13) $\left\|\Lambda^{s}(\lambda)\left[B\left(x, \partial_{x}, \lambda\right), P_{-}\left(x, \partial_{x}, \lambda\right)\right] h\right\| \leqq c\left\|\Lambda^{s-1}(\lambda) h\right\|$

14) $\left\|\Lambda^{s}(\lambda)\left[D\left(x, \partial_{x}, \lambda\right), E\left(x, \partial_{x}, \lambda\right)\right] h\right\| \leqq c \lambda^{-1}\left\|\Lambda^{s}(\lambda) h\right\|$

15) $\left\|\Lambda^{s}(\lambda)\left[E\left(x, \partial_{x}, \lambda\right), D\left(x, \partial_{x}, \lambda\right)\right] h\right\| \leqq c \lambda^{-1}\left\|\Lambda^{s}(\lambda) h\right\|$

16) $\left\|\left(i \eta-\Lambda^{1}(\lambda)\right)^{\gamma} \Lambda^{s-1}(\lambda)\left[M\left(x, \partial_{x}, \lambda\right), A^{-\gamma}\left(x, \partial_{x}, \eta, \lambda\right)\right] h\right\|$

$$
\leqq c\left\|\Lambda^{s-1}(\lambda) h\right\|
$$

17) $\left\|\left(i \eta-\Lambda^{1}(\lambda)\right)^{\gamma} \Lambda^{s-1}(\lambda)\left[A^{-\gamma}\left(x, \partial_{x}, \eta, \lambda\right), M\left(x, \partial_{x}, \lambda\right)\right] h\right\|$

$$
\leqq c\left\|\Lambda^{s-1}(\lambda) h\right\|
$$

18) $\left\{\int_{0}^{\infty} \| \Lambda^{s}(\lambda)\left\{\partial_{y}^{\gamma}\left(\left[M\left(x, \partial_{x}, \lambda\right), e^{\left.\left.\left.\left.-M\left(x, \partial_{x}, \lambda\right) y_{\circ} P_{-}\left(x, \partial_{x}, \lambda\right)\right]\right)\right\} h \|^{2} d y\right\}^{1 / 2}}\right.\right.\right.\right.$

$$
\leqq c\left\|\Lambda^{s+|\gamma|-1 / 2}(\lambda) h\right\|
$$

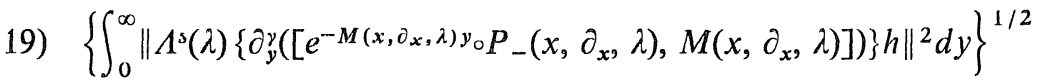

$$
\leqq c\left\|\Lambda^{s+|\gamma|-1 / 2}(\lambda) h\right\| \text {. }
$$

Remark. $\left[q\left(x, \partial_{x}\right), p\left(x, \partial_{x}\right)\right] \equiv q\left(x, \partial_{x}\right) \cdot p\left(x, \partial_{x}\right)-q\left(x, \partial_{x}\right) \circ p\left(x, \partial_{x}\right)$

Proof. 1) 12) follow easily from Lemmas 2.6, 2.7 and 3.8. From the definition of $[$,$] we have that$

$$
\left[D\left(x, \partial_{x}, \lambda\right), E\left(x, \partial_{x}, \lambda\right)\right]=-\sum_{1 \leqq|\alpha|<N}(i)^{|\alpha|}(\alpha !)^{-1} J_{\alpha}^{2}\left(x, \partial_{x}, \lambda\right)+K .
$$

Here, the error term $K$ is negligible to be estimated if $N$ is sufficiently large because $D$ and $E$ satisfy the hypotheses of Lemma 3.9. The other 
terms $J_{\alpha}^{2}$ satisfy the estimates 8) and 9) of this lemma. Thus we obtain 14). The other estimates are proved by the same way as 14).

End of proof.

Let $\varphi \in C_{0}^{\infty}\left(\overline{R_{+}^{n+1}}\right) ; \overline{R_{+}^{n+1}} \equiv\left\{(x, y) ; y \geqq 0,(x, y) \in R^{n+1}\right\}$. We define the operators $S_{1}(\lambda), S_{7}(\lambda)$ and $T_{11}(\lambda)$ as

$$
\begin{aligned}
& S_{1}(\lambda) \varphi \equiv \int e^{i y \eta} A^{-1}\left(x, \partial_{x}, \eta, \lambda\right) \hat{\varphi}(\eta) d \eta \\
& S_{7}(\lambda) \varphi \equiv\left[S_{1}(\lambda), M\left(x, \partial_{x}, \lambda\right)\right] \varphi \\
&=\int e^{i y \eta}\left[A^{-1}\left(x, \partial_{x}, \eta, \lambda\right), M\left(x, \partial_{x}, \lambda\right)\right] \hat{\varphi}(\eta) d \eta \\
& T_{11}(\lambda) \varphi \equiv\left[M\left(x, \partial_{x}, \lambda\right), S_{1}(\lambda)\right] \varphi \\
&=\int e^{i y \eta}\left[M\left(x, \partial_{x}, \lambda\right), A^{-1}\left(x, \partial_{x}, \eta, \lambda\right)\right] \hat{\varphi}(\eta) d \eta \\
& \hat{\varphi}(\eta) \equiv \int_{0}^{\infty} e^{-i \eta y} \varphi(\cdot, y) d y \text { and } A^{-1}(x, \xi, \eta, \lambda) \equiv\{i \eta+M(x, \xi, \lambda)\}^{-1} .
\end{aligned}
$$

Then, we have the following lemma.

Lemma 3.11. Let $\varphi \in C_{0}^{\infty}\left(\overline{R_{+}^{n+1}}\right)$. For any non-negative integer $m$,

1) $\left(\frac{\partial}{\partial y}\right)^{m+1} S_{1}(\lambda) \varphi$

$$
\begin{aligned}
=S_{1}(\lambda) & \cdot\left(\frac{\partial}{\partial y}\right)^{m+1} \varphi \\
& +\sum_{j=0}^{m}\left(\frac{\partial}{\partial y}\right)^{j} e^{-M\left(x, \partial_{x}, \lambda\right) y_{\circ} P_{-}\left(x, \partial_{x}, \lambda\right)\left(\left(\frac{\partial}{\partial y}\right)^{m-j} \varphi\right)(\cdot, 0)}
\end{aligned}
$$

2) $\left(\frac{\partial}{\partial y}\right)^{m+1} S_{7}(\lambda) \varphi$

$$
\begin{aligned}
& =S_{7}(\lambda) \cdot\left(\frac{\partial}{\partial y}\right)^{m+1} \varphi
\end{aligned}
$$

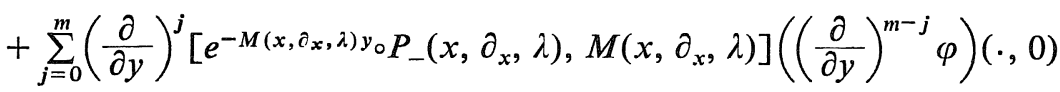


3) $\quad\left(\frac{\partial}{\partial y}\right)^{m+1} \cdot T_{11}(\lambda) \varphi=T_{11}(\lambda) \cdot\left(\frac{\partial}{\partial y}\right)^{m+1} \varphi$

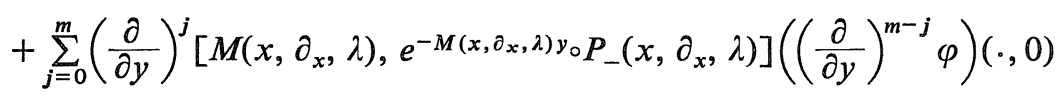

in $R_{+}^{n+1}$.

Proof. This is proved by using the relations that

$$
\begin{aligned}
& \int_{0}^{\infty} e^{-i \sigma t} \frac{\partial}{\partial t} f(t) d t=\int_{0}^{\infty} i \sigma e^{-i \sigma t} f(t) d t-f(0) \\
& \int_{-\infty}^{+\infty} e^{i s \sigma} \prod_{j=1}^{k}\left(i \sigma-\sigma_{j}\right)^{-1} d \sigma=(i)^{-1} \int_{\Gamma} e^{s \mu} \prod_{j=1}^{k}\left(\mu-\sigma_{j}\right)^{-1} d \mu
\end{aligned}
$$

where $s>0, \operatorname{Re} \sigma_{j} \neq 0$ and $\Gamma$ is a $C^{\infty}$-Jordan contour which surrounds $\sigma_{j}$ such that $\operatorname{Re} \sigma_{j}<0$.

End of proof.

Lemma 3.12. Let $\varphi \in C_{0}^{\infty}\left(\overline{R_{+}^{n+1}}\right)$. For any non-negative integer $m$,

1) $\sum_{|\alpha|+|\beta|+|\gamma|=m+1} c_{\alpha \beta \gamma}\left\|\lambda^{\alpha} \partial_{x}^{\beta} \partial_{y}^{\gamma} S_{1}(\lambda) \varphi\right\|_{+}^{2}$

$$
\leqq c \sum_{|\alpha|+|\beta|+|\gamma|=m} c_{\alpha \beta \gamma}\left\|\lambda^{\alpha} \partial_{x}^{\beta} \partial_{y}^{\gamma} \varphi\right\|_{+}^{2}
$$

2) $\sum_{|\alpha|+|\beta|+|\gamma|=m+1} c_{\alpha \beta \gamma}\left\|\lambda^{\alpha} \partial_{x}^{\beta} \partial_{y}^{\gamma} S_{7}(\lambda) \varphi\right\|_{+}^{2}$

$$
\leqq c_{|\alpha|+|\beta|+|\gamma|=m} c_{\alpha \beta \gamma}\left\|\lambda^{\alpha} \partial_{x}^{\beta} \partial_{y}^{\gamma} \varphi\right\|_{+}^{2}
$$

3) $\sum_{|\alpha|+|\beta|+|\gamma|=m+1} c_{\alpha \beta \gamma}\left\|\lambda^{\alpha} \partial_{x}^{\beta} \partial_{y}^{\gamma} T_{11}(\lambda) \varphi\right\|_{+}^{2}$

$$
\leqq c \sum_{|\alpha|+|\beta|+|\gamma|=m} c_{\alpha \beta \gamma}\left\|\lambda^{\alpha} \partial_{x}^{\beta} \partial_{y}^{\gamma} \varphi\right\|_{+}^{2}
$$

where $\|\varphi\|_{+}^{2} \equiv \iint_{R_{+}^{n+1}}|\varphi(x, y)|^{2} d x d y$.

Proof. We prove 1). When $m=0$, it follows from the non-singularity of $i \eta+M(x, \xi, \lambda)$, that is, from 12) of Lemma 3.10 ,

$$
\sum_{|\alpha|+|\beta|+|\gamma|=1}\left\|\lambda^{\alpha} \partial_{x}^{\beta} \partial_{y}^{\gamma} S_{1}(\lambda) \varphi\right\|_{+}^{2}
$$




$$
\begin{aligned}
& \leqq c \int\left\|\left(i \eta-\Lambda^{1}(\lambda)\right) A^{-1}\left(x, \partial_{x}, \eta, \lambda\right) \hat{\varphi}(\eta)\right\|^{2} d \eta \\
& \leqq c \int\|\hat{\varphi}(\eta)\|^{2} d \eta=c\|\varphi\|_{+}^{2} .
\end{aligned}
$$

We use Lemma 3.11 in the general case of $m$. The terms that $\gamma=0$ are estimated immediately by 12 ) of Lemma 3.10 , that is,

$$
\sum_{|\alpha|+|\beta|=m+1} c_{\alpha \beta}\left\|\lambda^{\alpha} \partial_{x}^{\beta} S_{1}(\lambda) \varphi\right\|_{+}^{2} \leqq c \sum_{|\alpha|+|\beta|=m} c_{\alpha \beta}\left\|\lambda^{\alpha} \partial_{x}^{\beta} \varphi\right\|_{+}^{2} .
$$

When $\gamma \neq 0$, we have by 1 ) of Lemma 3.11 that

$$
\begin{aligned}
& \left\|\lambda^{\alpha} \partial_{x}^{\beta} \partial_{y}^{\gamma} S_{1}(\lambda) \varphi\right\|_{+} \leqq\left\|\lambda^{\alpha} \partial_{x}^{\beta} \partial_{y}^{\gamma} S_{1}(\lambda) \partial_{y}^{\gamma-1} \varphi\right\|_{+} \\
& \quad+\sum_{j=1}^{\gamma-1} \| \lambda^{\alpha} \partial_{x}^{\beta} \partial_{y}^{\gamma-j} e^{-M\left(x, \partial_{x}, \lambda\right) y_{\circ} P_{-}\left(x, \partial_{x}, \lambda\right)\left(\partial_{y}^{j-1} \varphi\right)(\cdot, 0) \|_{+} \cdot}
\end{aligned}
$$

The first term is estimated by 12) of Lemma 3.10, too.

$$
\begin{aligned}
\left\|\lambda^{\alpha} \partial_{x}^{\beta} \partial_{y} S_{1}(\lambda) \partial_{y}^{\gamma-1} \varphi\right\|_{+}^{2} & \leqq c \int\left\|\Lambda^{|\alpha|+|\beta|}(\lambda) \widehat{\left(\partial_{y}^{\gamma-1} \varphi\right)}(\eta)\right\|^{2} d \eta \\
& \leqq c \sum_{|\alpha|+|\beta|=m-|\gamma|} c_{\alpha \beta \gamma}\left\|\lambda^{\alpha} \partial_{x}^{\beta} \partial_{y}^{\gamma} \varphi\right\|_{+}^{2} .
\end{aligned}
$$

We use 11) of Lemma 3.10 for the remained terms.

$$
\begin{aligned}
& \sum_{|\alpha|+|\beta|=m-|\gamma|+1} c_{\alpha \beta}\left\|\lambda^{\alpha} \partial_{x}^{\beta} \partial_{y}^{\gamma-j} e^{-M\left(x, \partial_{x}, \lambda\right) y_{\circ} P_{-}}\left(x, \partial_{x}, \lambda\right)\left(\partial_{y}^{j-1} \varphi\right)(\cdot, 0)\right\|_{+}^{2} \\
& \leqq c\left\|\Lambda^{|\alpha|+|\beta|+|\gamma|-j-1 / 2}(\lambda)\left(\partial_{y}^{j-1} \varphi\right)(\cdot, 0)\right\|^{2} \\
& \leqq c\left(\sum_{|\alpha|+|\beta|=m-|\gamma|} c_{\alpha \beta}\left\|\lambda^{\alpha} \partial_{x}^{\beta} \partial_{y}^{\gamma} \varphi\right\|_{+}^{2}+\sum_{|\alpha|+|\beta|=m-|\gamma|+1} c_{\alpha \beta}\left\|\lambda^{\alpha} \partial_{x}^{\beta} \partial_{y}^{\gamma-1} \varphi\right\|_{+}^{2}\right),
\end{aligned}
$$

where we use the well-known inequality that

$$
\left\|\Lambda^{1 / 2}(\lambda) \varphi(\cdot, 0)\right\|^{2} \leqq\left\|\Lambda^{1}(\lambda) \varphi\right\|_{+}^{2}+\left\|\partial_{y} \varphi\right\|_{+}^{2} .
$$

Thus, we obtain 1) of this lemma. We can prove 2) and 3) using 16) 19) of Lemma 3.10 in the same way as 1 ) because $S_{7}(\lambda)$ and $T_{11}(\lambda)$ have the similar expression 2) and 3) of Lemma 3.11. End of proof.

Lemma 3.13. Let $p(x, \xi, \lambda)$ satisfy the hypotheses of Lemma 3.7, where $0 \leqq \delta_{1} \leqq \rho_{1} \leqq 1$ and $\delta_{1} \neq 1$. Then we obtain 


$$
\left\|\Lambda^{m}(\lambda) p(x, \partial, \lambda) u\right\| \leqq c_{0} \lambda^{-m_{1}}\left\|\Lambda^{m+m_{1}}(\lambda) u\right\|+c(l, m)\left\|\Lambda^{l}(\lambda) u\right\|
$$

for $\lambda \in[1, \infty)$ and for any real number $l$ and $m$ such that $m+m_{1}>l$, where $c_{0}$ is a constant which does not depend on $\lambda, l$ and $m$.

Proof. This lemma is a corollary of Lemma 3.8. We know well the inequality that

$$
\left\|\Lambda^{\alpha}(\lambda) u\right\| \leqq \varepsilon\left\|\Lambda^{\alpha+\beta}(\lambda) u\right\|+\varepsilon^{(\gamma-\alpha) / \beta}\left\|\Lambda^{\gamma}(\lambda) u\right\|
$$

So we obtain

$$
\begin{aligned}
& \left\|\Lambda^{m+m_{1}+\delta_{1}-1}(\lambda) u\right\| \\
& \quad \leqq \varepsilon\left\|\Lambda^{m+m_{1}}(\lambda) u\right\|+\varepsilon^{\left(2 l-m-m_{1}-\delta_{1}+1\right) /\left(1-\delta_{1}\right)}\left\|\Lambda^{2 l}(\lambda) u\right\| \\
& \quad \leqq \varepsilon\left\|\Lambda^{m+m_{1}}(\lambda) u\right\|+c(l, m) \lambda^{m_{1}}\left\|\Lambda^{l}(\lambda) u\right\|
\end{aligned}
$$

where $\varepsilon=c_{0} / c_{m}$ and $l<\min \left(0, m_{1}\right)$. (It is no restriction that we assume it.)

End of proof.

Lemma 3.14. Let $\chi_{i} \in C_{0}^{\infty}\left(R^{n}\right)$ or $\chi_{i} \equiv 1(i=1,2)$ such that $0 \leqq \chi_{i} \leqq 1$ and $\chi_{2}=\chi_{2} \chi_{1}$. Let $p(x, \xi, \lambda)$ satisfy the hypotheses of Lemma 3.7, where $0 \leqq \delta_{1} \leqq \rho_{1} \leqq 1$ and $\delta_{1} \neq 1$. Then, we obtain that

$$
\begin{aligned}
& \left\|\Lambda^{m}(\lambda) \chi_{2} p(x, \partial, \lambda) u\right\| \leqq c_{0} \lambda^{-m_{1}}\left\|\Lambda^{m+m_{1}}(\lambda) \chi_{2} u\right\| \\
& \quad+c\left(l, m, \chi_{i}\right) \lambda^{-m_{1}-1+\rho_{1}}\left\|\Lambda^{m+m_{1}-\rho_{1}}(\lambda) \chi_{1} u\right\|+c\left(l, m, \chi_{i}\right)\left\|\Lambda^{l}(\lambda) u\right\|
\end{aligned}
$$

for $\lambda \in[1, \infty)$ and for any real number $l$ and $m$ such that $m+m_{1}>l$, where $c_{0}$ is a constant which does not depend on $\lambda, l, m$ and $\chi_{i}$.

Proof. In Lemma 3.9 we put $p(x, \partial, \lambda)=\chi_{2}(x), q(x, \partial, \lambda)=p(x, \partial, \lambda)$, $s=m$ and $m \leqq l$. Then we obtain that

$$
\begin{aligned}
& \left\|\Lambda^{m}(\lambda)\left\{p(x, \partial, \lambda) \chi_{2} u-\sum_{|\alpha|<N}(i)^{|\alpha|}(\alpha !)^{-1} \chi_{2(\alpha)} p^{(\alpha)}(x, \partial, \lambda) u\right\}\right\| \\
& \quad \leqq c\left(l, m, \chi_{2}\right)\left\|\Lambda^{l}(\lambda) u\right\| .
\end{aligned}
$$

We use again Lemma 3.9 for $p(x, \partial, \lambda)=\chi_{1}(x)$ and $q(x, \partial, \lambda)=\chi_{2(\alpha)} p^{(\alpha)}(x$, $\partial, \lambda)$ when $|\alpha| \geqq 1$. 


$$
\begin{aligned}
& \left\|\Lambda^{m}(\lambda)\left\{\chi_{2(\alpha)} p^{(\alpha)}(x, \partial, \lambda) \chi_{1} u-\chi_{2(\alpha)} p^{(\alpha)}(x, \partial, \lambda) u\right\}\right\| \\
& \quad \leqq c\left(l, m, \chi_{i}\right)\left\|\Lambda^{l}(\lambda) u\right\|
\end{aligned}
$$

because $\chi_{2(\alpha)} \chi_{1(\beta)} p^{(\alpha+\beta)}(x, \partial, \lambda)=0$, when $|\beta| \geqq 1$, and $\chi_{2(\alpha)} \chi_{1}=\chi_{2(\alpha)}$. Thus, we obtain that

$$
\begin{aligned}
& \left\|\Lambda^{m}(\lambda) \chi_{2} p(x, \partial, \lambda) u\right\| \leqq\left\|\Lambda^{m}(\lambda) p(x, \partial, \lambda) \chi_{2} u\right\| \\
& \quad+\left\|\Lambda^{m}(\lambda)\left\{\sum_{|\alpha|<N}(i)^{|\alpha|}(\alpha !)^{-1} \chi_{2(\alpha)} p^{(\alpha)}(x, \partial, \lambda)\right\} \chi_{1} u\right\| \\
& \quad+c\left(l, m, \chi_{i}\right)\left\|\Lambda^{l}(\lambda) u\right\| .
\end{aligned}
$$

We apply Lemma 3.13 to the first and second terms in the right hand side of the above inequality. Then, we obtain the result of Lemma 3.14 , because $\lambda^{|\alpha|} \chi_{2(\alpha)} p^{(\alpha)}(x, \partial, \lambda)(|\alpha| \geqq 1)$ satisfy the hypotheses of Lemma 3.7 for $p(x, \partial, \lambda)$ when we replace $m_{1}$ by $m_{1}-\rho_{1}|\alpha|$.

End of proof.

Using Lemma 3.14, we obtain the similar lemma as Lemma 3.10. This is used to prove the existence of regular solution or the regularity of solutions.

Lemma 3.15. Let $h$ be an element of $C_{0}^{\infty}\left(R^{n}\right), \lambda \in[1, \infty), m>l$ and $\chi_{i}(i=1,2)$ be these in Lemma 3.14. Then, there exists a constant $c_{0}$ which does not depend on $l, m, \lambda$ and $\chi_{i}$ such that

1) $\left\|\Lambda^{m}(\lambda) \chi_{2} M\left(x, \partial_{x}, \lambda\right) h\right\| \leqq c_{0}\left\|\Lambda^{m+1}(\lambda) \chi_{2} h\right\|$

$$
+c\left(l, m, \chi_{i}\right)\left\{\left\|\Lambda^{m}(\lambda) \chi_{1} h\right\|+\left\|\Lambda^{l}(\lambda) h\right\|\right\}
$$

2) $\left\|\Lambda^{m}(\lambda) \chi_{2} B\left(x, \partial_{x}, \lambda\right) h\right\| \leqq c_{0}\left\|\Lambda^{m}(\lambda) \chi_{2} h\right\|$

$$
+c\left(l, m, \chi_{i}\right)\left\{\left\|\Lambda^{m-1}(\lambda) \chi_{1} h\right\|+\left\|\Lambda^{l}(\lambda) h\right\|\right\}
$$

3) $\left\|\Lambda^{m}(\lambda) \chi_{2} P_{-}\left(x, \partial_{x}, \lambda\right) h\right\| \leqq c_{0}\left\|\Lambda^{m}(\lambda) \chi_{2} h\right\|$

$$
+c\left(l, m, \chi_{i}\right)\left\{\left\|\Lambda^{m-1}(\lambda) \chi_{1} h\right\|+\left\|\Lambda^{l}(\lambda) h\right\|\right\}
$$

4) $\left\|\Lambda^{m}(\lambda) \chi_{2} D\left(x, \partial_{x}, \lambda\right) h\right\| \leqq c_{0}\left\|\Lambda^{m}(\lambda) \chi_{2} h\right\|$

$$
+c\left(l, m, \chi_{i}\right)\left\{\left\|\Lambda^{m-1}(\lambda) \chi_{1} h\right\|+\left\|\Lambda^{l}(\lambda) h\right\|\right\}
$$


5) $\left\|\Lambda^{m}(\lambda) \chi_{2} E\left(x, \partial_{x}, \lambda\right) h\right\| \leqq c_{0} \lambda^{-1}\left\|\Lambda^{m+1}(\lambda) \chi_{2} h\right\|$

$$
+c\left(l, m, \chi_{i}\right)\left\{\lambda^{-3 / 2}\left\|\Lambda^{m+1 / 2}(\lambda) \chi_{1} h\right\|+\left\|\Lambda^{l}(\lambda) h\right\|\right\}
$$

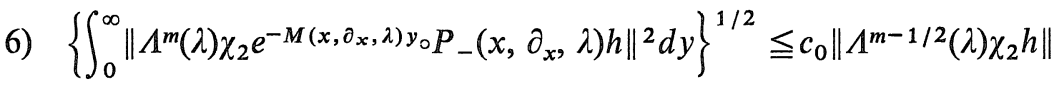

$$
+c\left(l, m, \chi_{i}\right)\left\{\left\|\Lambda^{m-3 / 2}(\lambda) \chi_{1} h\right\|+\left\|\Lambda^{l}(\lambda) h\right\|\right\}
$$

7) $\left\|\left(i \eta-\Lambda^{1}(\lambda)\right) \Lambda^{m-1}(\lambda) \chi_{2} A^{-1}\left(x, \partial_{x}, \eta, \lambda\right) h\right\| \leqq c_{0}\left\|\Lambda^{m-1}(\lambda) \chi_{2} h\right\|$

$$
+c\left(l, m, \chi_{i}\right)\left\{\left\|\Lambda^{m-2}(\lambda) \chi_{1} h\right\|+\left\|\Lambda^{l}(\lambda) h\right\|\right\}
$$

8) $\left\|\Lambda^{m}(\lambda) \chi_{2}\left[B\left(x, \partial_{x}, \lambda\right), P_{-}\left(x, \partial_{x}, \lambda\right)\right] h\right\| \leqq c_{0}\left\|\Lambda^{m-1}(\lambda) \chi_{2} h\right\|$

$$
+c\left(l, m, \chi_{i}\right)\left\{\left\|\Lambda^{m-2}(\lambda) \chi_{1} h\right\|+\left\|\Lambda^{l}(\lambda) h\right\|\right\}
$$

9) $\left\|\Lambda^{m}(\lambda) \chi_{2}\left[D\left(x, \partial_{x}, \lambda\right), E\left(x, \partial_{x}, \lambda\right)\right] h\right\| \leqq c_{0} \lambda^{-1}\left\|\Lambda^{m}(\lambda) \chi_{2} h\right\|$

$$
+c\left(l, m, \chi_{i}\right)\left\{\lambda^{-3 / 2}\left\|\Lambda^{m-1 / 2}(\lambda) \chi_{1} h\right\|+\left\|\Lambda^{l}(\lambda) h\right\|\right\}
$$

10) $\left\|\Lambda^{m}(\lambda) \chi_{2}\left[E\left(x, \partial_{x}, \lambda\right), D\left(x, \partial_{x}, \lambda\right)\right] h\right\| \leqq c_{0} \lambda^{-1}\left\|\Lambda^{m}(\lambda) \chi_{2} h\right\|$

$$
+c\left(l, m, \chi_{i}\right)\left\{\lambda^{-3 / 2}\left\|\Lambda^{m-1 / 2}(\lambda) \chi_{1} h\right\|+\left\|\Lambda^{l}(\lambda) h\right\|\right\}
$$

11) $\left\|\left(i \eta-\Lambda^{1}(\lambda)\right) \Lambda^{m-1}(\lambda) \chi_{2}\left[A^{-1}\left(x, \partial_{x}, \eta, \lambda\right), M\left(x, \partial_{x}, \lambda\right)\right] h\right\|$

$$
\leqq c_{0}\left\|\Lambda^{m-1}(\lambda) \chi_{2} h\right\|+c\left(l, m, \chi_{i}\right)\left\{\left\|\Lambda^{m-2}(\lambda) \chi_{1} h\right\|+\left\|\Lambda^{l}(\lambda) h\right\|\right\}
$$

12) $\left\|\left(i \eta-\Lambda^{1}(\lambda)\right) \Lambda^{m-1}(\lambda) \chi_{2}\left[M\left(x, \partial_{x}, \lambda\right), A^{-1}\left(x, \partial_{x}, \eta, \lambda\right)\right] h\right\|$

$$
\leqq c_{0}\left\|\Lambda^{m-1}(\lambda) \chi_{2} h\right\|+c\left(l, m, \chi_{i}\right)\left\{\left\|\Lambda^{m-2}(\lambda) \chi_{1} h\right\|+\left\|\Lambda^{l}(\lambda) h\right\|\right\}
$$

13) $\left\{\int_{0}^{\infty} \| \Lambda^{m}(\lambda) \chi_{2}\left[M\left(x, \partial_{x}, \lambda\right), e^{\left.\left.-M\left(x, \partial_{x}, \lambda\right) y_{0} P_{-}\left(x, \partial_{x}, \lambda\right)\right] h \|^{2} d y\right\}^{1 / 2}}\right.\right.$

$$
\leqq c_{0}\left\|\Lambda^{m-1 / 2}(\lambda) \chi_{2} h\right\|+c\left(l, m, \chi_{i}\right)\left\{\left\|\Lambda^{m-3 / 2}(\lambda) \chi_{1} h\right\|+\left\|\Lambda^{l}(\lambda) h\right\|\right\}
$$

14) $\left\{\int_{0}^{\infty} \| \Lambda^{m}(\lambda) \chi_{2}\left[e^{\left.\left.-M\left(x, \partial_{x}, \lambda\right) y_{\circ} P_{-}\left(x, \partial_{x}, \lambda\right), M\left(x, \partial_{x}, \lambda\right)\right] h \|^{2} d y\right\}^{1 / 2}}\right.\right.$

$$
\leqq c_{0}\left\|\Lambda^{m-1 / 2}(\lambda) \chi_{2} h\right\|+c\left(l, m, \chi_{i}\right)\left\{\left\|\Lambda^{m-3 / 2}(\lambda) \chi_{1} h\right\|+\left\|\Lambda^{l}(\lambda) h\right\|\right\} \text {. }
$$

We say finally some properties about a special type of pseudo-differ- 
ential operators in $R_{+}^{n+1}$. One of them has the symbol $(|\zeta|-i \eta)^{\alpha}$, which will appear in the lower terms and in the translators when we shall adapt the general cases to the special cases at the section 6 . It is a typical example of pseudo-differential operators satisfying the transmission property with which L. Boutet de Monvel has dealt in [3]. We may be able to deal with boundary value problems of these types of pseudo-differential operators. But we use only the special ones and it is sufficient as far as we deal with the boundary value problems of partial differential operators in this paper. 12), 16) and 17) of Lemma 3.10 show that $\Lambda^{1} \cdot A^{-1}, \Lambda^{1} \cdot\left[M, A^{-1}\right]$ and $\Lambda^{1} \cdot\left[A^{-1}, M\right]$ are ones of the simplest symbols of pseudo-differential operators in $y \in R^{1}$, which take their values in $B L\left(H^{s}\left(R^{n}\right)\right)$, because $\partial_{\eta}^{\alpha} A^{-1}=A^{-\alpha}, \partial_{\eta}^{\alpha}\left[M, A^{-1}\right]=\left[M, A^{-\alpha}\right]$ and $\partial_{\eta !}^{\alpha}\left[A^{-1}, M\right]=\left[A^{-\alpha}, M\right]$. We generalize them in the case that they depend on the variable $y$. Let $M(x, y, \xi, \lambda)$ be a $C^{\infty}$-function, which is constant in $y$ outside a bounded set. We assume that it satisfies the conditions of $M(x, \xi, \lambda)$ for each fixed $y$. We can define $A_{2}^{-\alpha}\left(x, y, \partial_{x}\right.$, $\eta, \lambda)$ with the symbol $A_{2}^{-\alpha}(x, y, \xi, \eta, \lambda) \equiv\{i \eta+M(x, y, \xi, \lambda)\}^{-\alpha}$, also $[M$, $\left.A_{2}^{-\alpha}\right]$ and $\left[A_{2}^{-\alpha}, M\right]$, where the bracket $[$,$] is the one as in the$ pseudo-differential operator in $x$. We have Lemma 3.16 as well as 3.10 .

Lemma 3.16. For $h \in C_{0}^{\infty}\left(R^{n}\right)$

1) $\left\|\left(i \eta-\Lambda^{1}(\lambda)\right)^{\alpha} \Lambda^{m}(\lambda) \partial_{y}^{\beta} A_{2}^{-\alpha}\left(x, y, \partial_{x}, \eta, \lambda\right) h\right\| \leqq c\left\|\Lambda^{m}(\lambda) h\right\|$

2) $\left\|\left(i \eta-\Lambda^{1}(\lambda)\right)^{\alpha} \Lambda^{m}(\lambda) \partial_{y}^{\beta}\left[M\left(x, y, \partial_{x}, \lambda\right), A_{2}^{-\alpha}\left(x, y, \partial_{x}, \eta, \lambda\right)\right] h\right\|$

$$
\leqq c\left\|\Lambda^{m}(\lambda) h\right\|
$$

3) $\left\|\left(i \eta-\Lambda^{1}(\lambda)\right)^{\alpha} \Lambda^{m}(\lambda) \partial_{y}^{\beta}\left[A_{2}^{-\alpha}\left(x, y, \partial_{x}, \eta, \lambda\right), M\left(x, y, \partial_{x}, \lambda\right)\right] h\right\|$

$$
\leqq c\left\|\Lambda^{m}(\lambda) h\right\| \text {. }
$$

(We omit the proof.)

This shows us that they have stronger properties, for example, $A_{2}^{-\alpha}$ takes its value in $B L\left(H^{s}\left(R^{n}\right), H^{s+\alpha}\left(R^{n}\right)\right)$. So, we modify Lemmas 3.2 and 3.6 in order to make a good use of them. 
Lemma 3.17. Let $Y$ be another Hilbert space densely contained in $X$ by a continuous injection. Let $p(x, \xi)$ and $q(x, \xi)$ be ones in Lemma 3.2 or 3.6 , and let $q(x, \xi)$ satisfy that there exists an integer $\alpha_{0}$ such that $q_{(\beta)}^{(\alpha)}(x, \xi) \in B L(X, Y)$ (bounded linear operators from $X$ to $Y$ ) and

$$
\left|q_{(\beta)}^{(\alpha)}(x, \xi)\right|_{X Y} \leqq c_{\alpha \beta}(1+|\xi|)^{m_{3}-\rho_{2}|\alpha|+\delta_{2}|\beta|}
$$

for all $|\alpha| \geqq \alpha_{0}$ and $|\beta| \geqq 0$, where $|\cdot|_{X Y}$ is the norm of $B L(X, Y)$. Then, there exist $N$ and $c$ for any integer $m$ such that, for $u \in C_{0}^{\infty}\left(R^{n}, X\right)$,

$$
q(x, \partial) \cdot p(x, \partial) u-\sum_{|\alpha|<N}(i)^{|\alpha|}(\alpha !)^{-1} q^{(\alpha)}(x, \partial) \circ p_{(\alpha)}(x, \partial) u=K u
$$

and $\|K u\|_{Y^{0}} \leqq c\|u\|_{X^{m}}$. Here $\|\cdot\|_{X^{m}}$ and $\|\cdot\|_{Y^{m}}$ stand for the norms of $H^{m}\left(R^{n}, X\right)$ and $H^{m}\left(R^{n}, Y\right)$, respectively。

Proof. We have only to replace the norm $|\cdot|$ of $B L(X)$ by the norm $|\cdot|_{X Y}$ of $B L(X, Y)$ for the estimation of $\hat{r}^{(\alpha)}(\zeta, \xi, \eta-\xi)$ in Lemma 3.3 or $\hat{r}^{(\alpha)}(\xi, \eta-\xi)$ in Lemma 3.6 .

End of proof.

We immediately obtain Lemma 3.18 by applying Lemmas 3.1 and 3.17 .

Lemma 3.18. Let $\chi_{i}(x, y) \in C^{\infty}\left(R^{n+1}\right)$ such that $\chi_{i} \equiv 1$ or $\in C_{0}^{\infty}\left(R^{n+1}\right)$, $0 \leqq \chi_{i} \leqq 1$ and $\chi_{2} \chi_{1}=\chi_{2}$. Let $\lambda \geqq 1, \alpha, \beta, \gamma$ integers and $s, t, \sigma, \tau$ real numbers. Then, we obtain the following estimates for all $u \in C_{0}^{\infty}\left(R^{n+1}\right)$, where $\|\cdot\|$ stands for the standard $L^{2}$-norm on $R^{n+1}$ and $c_{0}$ is a constant not depending on $s, t, \sigma, \tau$ and $\lambda$. We set

$$
\begin{aligned}
& A_{2(\beta)}^{-\alpha}(x, y, \xi, \eta, \lambda) \equiv \partial_{y}^{\beta} A_{2}^{-\alpha}(x, y, \xi, \eta, \lambda) \\
& {\left[M, A_{2}^{-\alpha}\right]_{(\beta)}\left(x, y, \partial_{x}, \eta, \lambda\right) \equiv \partial_{y}^{\beta}\left[M\left(x, y, \partial_{x}, \lambda\right), A_{2}^{-\alpha}\left(x, y, \partial_{x}, \eta, \lambda\right)\right]} \\
& {\left[A_{2}^{-\alpha}, M\right]_{(\beta)}\left(x, y, \partial_{x}, \eta, \lambda\right) \equiv \partial_{y}^{\beta}\left[A_{2}^{-\alpha}\left(x, y, \partial_{x}, \eta, \lambda\right), M\left(x, y, \partial_{x}, \lambda\right)\right] .}
\end{aligned}
$$

1) $\left\|\left(\Lambda^{1}(\lambda)-i \partial_{y}\right)^{\alpha} \cdot \Lambda^{s}(\lambda) \cdot A_{2(\beta)}^{-\alpha}\left(x, y, \partial_{x}, \partial_{y}, \lambda\right) u\right\| \leqq c\left\|\Lambda^{s}(\lambda) u\right\|$

2) $\left\|\left(\Lambda^{1}(\lambda)-i \partial_{y}\right)^{\alpha} \cdot \Lambda^{s}(\lambda) \cdot\left[M, A_{2}^{-\alpha}\right]_{(\beta)}\left(x, y, \partial_{x}, \partial_{y}, \lambda\right) u\right\| \leqq c\left\|\Lambda^{\alpha}(\lambda) u\right\|$

3) $\left\|\left(\Lambda^{1}(\lambda)-i \partial_{y}\right)^{\alpha} \cdot \Lambda^{s}(\lambda) \cdot\left[A_{2}^{-\alpha}, M\right]_{(\beta)}\left(x, y, \partial_{x}, \partial_{y}, \lambda\right) u\right\| \leqq c\left\|\Lambda^{\alpha}(\lambda) u\right\|$ 
4) $\left\|\Lambda^{s}(\lambda) \cdot\left(\Lambda^{1}(\lambda)-i \partial_{y}\right)^{\sigma} \cdot \chi_{2} \cdot\left(\Lambda^{1}(\lambda)-i \partial_{y}\right)^{-\sigma} u\right\|$

$$
\leqq\left\|\Lambda^{s}(\lambda) \chi_{2} u\right\|+c\left\{\left\|\Lambda^{s}(\lambda) \cdot\left(\Lambda^{1}(\lambda)-i \partial_{y}\right)^{-1} \chi_{1} u\right\|+\left\|\Lambda^{\tau}(\lambda) u\right\|\right\}
$$

5) $\quad\left\|\left(\Lambda^{1}(\lambda)-i \partial_{y}\right) \cdot \Lambda^{s}(\lambda) \cdot \chi_{2} \cdot A_{2}^{-1}\left(x, y, \partial_{x}, \partial_{y}, \lambda\right) u\right\| \leqq c_{0}\left\|\Lambda^{s}(\lambda) \cdot \chi_{2} u\right\|$

$$
+c\left\{\left\|\Lambda^{s-1}(\lambda) \cdot \chi_{1} u\right\|+\left\|\Lambda^{\tau}(\lambda) u\right\|\right\}
$$

6) $\left\|\left(\Lambda^{1}(\lambda)-i \partial_{y}\right) \cdot \Lambda^{s}(\lambda) \cdot \chi_{2} \cdot\left[M, A_{2}^{-1}\right]_{(0)}\left(x, y, \partial_{x}, \partial_{y}, \lambda\right) u\right\|$

$$
\leqq c_{0}\left\|\Lambda^{s}(\lambda) \cdot \chi_{2} u\right\|+c\left\{\left\|\Lambda^{s-1}(\lambda) \cdot \chi_{1} u\right\|+\left\|\Lambda^{\tau}(\lambda) u\right\|\right\}
$$

7) $\left\|\left(\Lambda^{1}(\lambda)-i \partial_{y}\right) \cdot \Lambda^{s}(\lambda) \cdot \chi_{2} \cdot\left[A_{2}^{-1}, M\right]_{(0)}\left(x, y, \partial_{x}, \partial_{y}, \lambda\right) u\right\|$

$$
\leqq c_{0}\left\|\Lambda^{s}(\lambda) \cdot \chi_{2} u\right\|+c\left\{\left\|\Lambda^{s-1}(\lambda) \cdot \chi_{1} u\right\|+\left\|\Lambda^{\tau}(\lambda) u\right\|\right\}
$$

Proof. We give the proof of (5). We first consider the commutation of $\chi_{2}$ and $A_{2}^{-1}\left(x, y, \partial_{x}, \partial_{y}, \lambda\right)$ in the variable $y$. We have (3.11) by Lemma 3.17 as well as we have had the similar one in the proof of Lemma 3.14.

$$
\begin{aligned}
& A_{2}^{-1}\left(x, y, \partial_{\lambda}, \partial_{y}, \lambda\right) \cdot \chi_{2}(x, y) v \\
& \quad-\sum_{v=0}^{N} c_{v} A_{2}^{-1(v)}\left(x, y, \partial_{x}, \partial_{y}, \lambda\right) \underset{(y)}{\circ} \chi_{2(v)}(x, y) v \\
& \quad=K v, \quad v \in C_{0}^{\infty}\left(R^{n+1}\right)
\end{aligned}
$$

and

$$
\left\|\left(\Lambda^{1}(\lambda)-i \partial_{y}\right) \Lambda^{s}(\lambda) K v\right\| \leqq c\left\|\Lambda^{\tau}(\lambda) v\right\|
$$

for a sufficiently large $N$, where $\underset{(y)}{\circ}$ stands for the product in the sense of symbols as pseudo-differential operators in $y$ valued in pseudo-differential operators in $x$. By definition $A_{2}^{-1(v)}\left(x, y, \partial_{x}, \partial_{y}, \lambda\right)_{(y)}^{\circ} \chi_{2(v)}(x, y)$ have the symbols $A_{2}^{-1(v)}\left(x, y, \partial_{x}, \eta, \lambda\right) \cdot \chi_{2(v)}(x, y)$. So we apply it Lemma 3.14 as pseudo-differential operators in $x$. We have for a sufficiently large $N$

$$
\begin{aligned}
& A_{2}^{-1(v)}\left(x, y, \partial_{x}, \eta, \lambda\right) \cdot \chi_{2(v)}(x, y) h \\
& \quad-\sum_{|\alpha|<N} \chi_{2(v)(\alpha)}(x, y) A_{2}^{-1(v)(\alpha)}\left(x, y, \partial_{x}, \eta, \lambda\right) h
\end{aligned}
$$




$$
=K^{\prime}(y, \eta, \lambda) h, \quad h \in C_{0}^{\infty}\left(R^{n}\right)
$$

and

$$
\left\|\Lambda^{s}(\lambda) \partial_{y}^{\alpha} \partial_{\eta}^{\beta} K^{\prime}(y, \eta, \lambda) h\right\| \leqq c_{\alpha \beta s \tau}(1+|\eta|)^{-\beta-v-1}\left\|\Lambda^{\tau}(\lambda) h\right\|,
$$

where $N$ does not depend on $\alpha$ and $\beta$, because

$$
\begin{aligned}
& \left|\partial_{x}^{\gamma} \partial_{\xi}^{\delta} \partial_{y}^{\alpha} \partial_{\eta}^{\beta} A_{2}^{-1(v)}(x, y, \xi, \eta, \lambda)\right| \\
& \quad \leqq c_{\alpha \beta \gamma \delta v}(1+|(\xi, \eta, \lambda)|)^{-v-\beta-|\delta|-1} \\
& \quad \leqq c_{\alpha \beta \gamma \delta v}(1+|\eta|)^{-v-\beta-1}(1+|(\xi, \lambda)|)^{-|\delta|} .
\end{aligned}
$$

Therefore we have for $u \in C_{0}^{\infty}\left(R^{n+1}\right)$

$$
\left\|\left(\Lambda^{1}(\lambda)-i \partial_{y}\right) \cdot \Lambda^{s}(\lambda) \cdot K^{\prime}\left(y, \partial_{y}, \lambda\right) u\right\| \leqq c_{s \tau}\left\|\Lambda^{\tau}(\lambda) u\right\| .
$$

This means that

$$
\begin{aligned}
& A_{2}^{-1}\left(x, y, \partial_{x}, \partial_{y}, \lambda\right) \cdot \chi_{2}(x, y) v \\
& \quad-\sum_{v<N} \sum_{|\alpha|<N} \chi_{2(v)(\alpha)}(x, y) A_{2}^{-1(v)(\alpha)}\left(x, y, \partial_{x}, \partial_{y}, \lambda\right) v \\
& \quad \equiv K_{0} v, \quad v \in C_{0}^{\infty}\left(R^{n+1}\right)
\end{aligned}
$$

and

$$
\left\|\left(\Lambda^{1}(\lambda)-i \partial_{y}\right) \cdot \Lambda^{s}(\lambda) \cdot K_{0} v\right\| \leqq c\left\|\Lambda^{\tau}(\lambda) v\right\|
$$

We commute $\chi_{1}$ and each term at the second part of the left hand side to modify $v$ by $\chi_{1}$ except for the principal term

$$
\chi_{2}(x, y) A_{2}^{-1}\left(x, y, \partial_{x}, \partial_{y}, \lambda\right) v
$$

Then, we have that

$$
\begin{aligned}
& A_{2}^{-1} \cdot \chi_{2} v-\chi_{2} \cdot A_{2}^{-1} v-\sum_{\substack{v<N \\
|\alpha|<N \\
v, \alpha) \neq 0}} \chi_{2(v)(\alpha)} \cdot A_{2}^{-1(v)(\alpha)} \cdot \chi_{1} v \\
& \quad=K_{1} v
\end{aligned}
$$

and $\left\|\left(\Lambda^{1}(\lambda)-i \partial_{y}\right) \cdot \Lambda^{s}(\lambda) K_{1} v\right\| \leqq c\left\|\Lambda^{\tau}(\lambda) v\right\|$, where it is clear that $\chi_{2(v)(\alpha)}{ }^{\circ}$ 
$A_{2}^{-1(v)(\alpha)}$ satisfy the estimates

$$
\begin{gathered}
\left\|\left(\Lambda^{1}(\lambda)-i \partial_{y}\right) \cdot \Lambda^{s}(\lambda) \chi_{2(v)(\alpha)} \cdot A_{2}^{-1(v)(\alpha)} v\right\| \\
\leqq c_{v s}\left\|\Lambda^{s-|\alpha|}(\lambda) \cdot\left(\Lambda^{1}(\lambda)-i \partial_{y}\right)^{-v} v\right\| .
\end{gathered}
$$

Thus we obtain the estimate 5). The other estimates 4), 6) and 7) can be analogically proved.

End of proof.

The operators $\left(\Lambda^{1}(\lambda) \pm i \partial_{y}\right)^{s}$ are isomorphisms from $H^{s+t}\left(R^{n+1}\right)$ to $H^{t}\left(R^{n+1}\right)$ for real numbers $s$ and $t$ and the symbols of $\left(\Lambda^{1}(\lambda) \pm i \partial_{y}\right)^{s}$ are $(|\zeta| \pm i \eta)^{s} ; \zeta=(\xi, \lambda)$, where we take the branch of $z^{s}$ for $\operatorname{Re} z \geqq 0$ which is real if $z$ is real. They are the adjoint operators to each other on $H^{0}\left(R^{n+1}\right) . \quad\left(\Lambda^{1}(\lambda) \pm i \partial_{y}\right)^{s} u=0 \quad$ in $\quad R_{+}^{n+1} \quad$ if $u \in H^{t}\left(R^{n+1}\right)(t \geqq 0, s+t \geqq 0)$ and if $u=0$ in $R_{+}^{n+1}$. Moreover $y^{-1 / 2}\left(\Lambda^{1}(\lambda) \pm i \partial_{y}\right)^{-1 / 2} u \in H^{0}\left(R_{ \pm}^{n+1}\right)$ if $u \in H^{0}\left(R^{n+1}\right)$ and if $u=0$ in $R_{+}^{n+1}$. So, $\left(\Lambda^{1}(\lambda)-i \partial_{y}\right)^{s}$ induces the isomorphism from $H^{s+t}\left(R_{+}^{n+1}\right)$ to $H^{t}\left(R_{+}^{n+1}\right)$ and $\left(\Lambda^{1}(\lambda)+i \partial_{y}\right)^{s}$ the isomorphism from $H_{0}^{s+t}\left(R_{+}^{n+1}\right)$ to $H_{0}^{t}\left(R_{+}^{n+1}\right)$ if $s+t$ and $t$ are positive, where $H_{0}^{t}\left(R_{+}^{n+1}\right)$ are the closed subspace of $H^{t}\left(R_{+}^{n+1}\right)$ densely containing $C_{0}^{\infty}\left(R_{+}^{n+1}\right)$ if $t$ are non-negative integers and the others are defined by the interpolation of them.

$\left(\Lambda^{1}(\lambda)+i \partial_{y}\right)^{s *}$ the adjoint operator of $\left(\Lambda^{1}(\lambda)+i \partial_{y}\right)^{s}$ on $H_{0}^{t}\left(R_{+}^{n+1}\right)$ is the isomorphism from $H^{s-t}\left(R_{+}^{n+1}\right)$ to $H^{-t}\left(R_{+}^{n+1}\right)$ if $s-t$ and $-t$ are non-positive and it is equal to $\left(\Lambda^{1}(\lambda)-i \partial_{y}\right)^{s}$ on $C_{0}^{\infty}\left(\overline{R_{+}^{n+1}}\right)$. So we denote again it by $\left(\Lambda^{1}(\lambda)-i \partial_{y}\right)^{s}$. It has the representation that for $\varphi \in C_{0}^{\infty}\left(\overline{R_{+}^{n+1}}\right)$

$$
\begin{aligned}
\left(\Lambda^{1}(\lambda)-i \partial_{y}\right)^{s} \varphi & =\iint e^{i(x \xi+y \eta)}(|\zeta|-i \eta)^{s} \hat{\varphi}(\xi, \eta) d \xi d \eta \\
\hat{\varphi} & =\int_{R^{n}} d x \int_{0}^{\infty} e^{-i(\xi x+\eta y)} \varphi(x, y) d y
\end{aligned}
$$

It is clear that $\left(\Lambda^{1}(\lambda)-i \partial_{y}\right)^{s}\left(\Lambda^{1}(\lambda)-i \partial_{y}\right)^{t}=\left(\Lambda^{1}(\lambda)-i \partial_{y}\right)^{s+t}$. Therefore we obtain Lemma 3.19 .

Lemma 3.19. $\left(\Lambda^{1}(\lambda)-i \partial_{y}\right)^{s}$ induces the isomorphism from $H^{s+t}\left(R_{+}^{n+1}\right)$ to $H^{t}\left(R_{+}^{n+1}\right)$ for all real number $s$ and $t$ and its inverse is $\left(\Lambda^{1}(\lambda)\right.$ $\left.-i \partial_{y}\right)^{-s}$. Let $\chi_{i}(i=1,2)$ be $C^{\infty}$-functions defined in Lemma 3.18. Then we have for all real number $s, \sigma$ and $\tau$ and for all $u \in C_{0}^{\infty}\left(\overline{R_{+}^{n+1}}\right)$ 


$$
\begin{aligned}
& \left\|\Lambda^{s}(\lambda) \cdot\left(\Lambda^{1}(\lambda)-i \partial_{y}\right)^{\sigma} \cdot \chi_{2} \cdot\left(\Lambda^{1}(\lambda)-i \partial_{y}\right)^{-\sigma} u\right\|_{+} \\
& \quad \leqq\left\|\Lambda^{s}(\lambda) \cdot \chi_{2} u\right\|_{+}+c\left\{\left\|\Lambda^{s}(\lambda) \cdot\left(\Lambda^{1}(\lambda)-i \partial_{y}\right)^{-1} \chi_{1} u\right\|_{+}+\left\|\Lambda^{\tau}(\lambda) u\right\|_{+}\right\}
\end{aligned}
$$

Proof. In the same way of (3.12) we have

$$
\begin{aligned}
\chi_{2} v & -\left(\Lambda^{1}(\lambda)-i \partial_{y}\right)^{\sigma} \cdot \chi_{2} \cdot\left(\Lambda^{1}(\lambda)-i \partial_{y}\right)^{-\sigma} v \\
& -\sum_{v=1}^{N} a_{v}\left(x, y, \partial_{x}, \lambda\right) \cdot\left(\Lambda^{1}(\lambda)-i \partial_{y}\right)^{-v} \cdot \chi_{1} v=K v
\end{aligned}
$$

where $a_{v}\left(x, y, \partial_{x}, \lambda\right)$ are pseudo-differential operators in $x$ such that $\left\|\Lambda^{s}(\lambda) \cdot a_{v}\left(x, y, \partial_{x}, \lambda\right) \cdot h\right\| \leqq c\left\|\Lambda^{s+v-1}(\lambda) h\right\|$ for $h \in C_{0}^{\infty}\left(R^{n}\right)$ and $K$ is the error term such that $\left\|\Lambda^{s}(\lambda) K v\right\| \leqq c\left\|\Lambda^{\tau}(\lambda) v\right\|$ for $v \in C_{0}^{\infty}\left(R^{n+1}\right)$. Thus (3.13) holds for $\tilde{u}=u$ on $\overline{R_{+}^{n+1}}$ and $\tilde{u}=0$ on $R_{-}^{n+1}$, where $u \in C_{0}^{\infty}\left(\overline{R_{+}^{n+1}}\right)$. We obtain the estimate of the lemma if we take the norm \|\|$_{+}$in $R_{+}^{n+1}$.

End of proof.

Lemma 3.20. Let $\chi_{i}(i=1,2)$ be $C^{\infty}$-functions defined in Lemma 3.18 and $\chi_{i}^{0}(x) \equiv \chi_{i}(x, 0)$. We obtain for $h \in C_{0}^{\infty}\left(R^{n}\right)$ and for all real number $s$ and $\tau$

1) $\left\|\Lambda^{s}(\lambda) \chi_{2} e^{-\Lambda(\lambda) y} h\right\|_{+} \leqq c_{0}\left\|\Lambda^{s-1 / 2}(\lambda) \chi_{2}^{0} h\right\|$

$$
+c\left(s, \tau, \chi_{i}\right)\left\{\left\|\Lambda^{s-3 / 2}(\lambda) \chi_{1}^{0} h\right\|+\left\|\Lambda^{\tau}(\lambda) h\right\|\right\}
$$

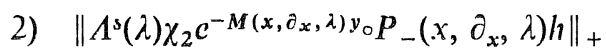

$$
\leqq c_{0}\left\|\Lambda^{s-1 / 2}(\lambda) \chi_{2}^{0} h\right\|+c\left(s, \tau, \chi_{i}\right)\left\{\left\|\Lambda^{s-3 / 2}(\lambda) \chi_{1}^{0} h\right\|+\left\|\Lambda^{\mathrm{r}}(\lambda) h\right\|\right\}
$$

3) $\| \Lambda^{s}(\lambda) \chi_{2}\left[M\left(x, \partial_{x}, \lambda\right), e^{\left.-M\left(x, \partial_{x}, \lambda\right) y_{\circ} P_{-}\left(x, \partial_{x}, \lambda\right)\right] h \|_{+}}\right.$

$$
\leqq c_{0}\left\|\Lambda^{s-1 / 2}(\lambda) \chi_{2}^{0} h\right\|+c\left(s, \tau, \chi_{i}\right)\left\{\left\|\Lambda^{s-3 / 2}(\lambda) \chi_{1}^{0} h\right\|+\left\|\Lambda^{\tau}(\lambda) h\right\|\right\}
$$

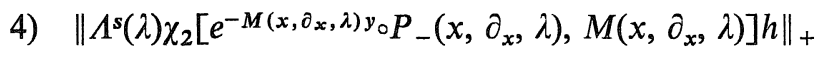

$$
\leqq c_{0}\left\|\Lambda^{s-1 / 2}(\lambda) \chi_{2}^{0} h\right\|+c\left(s, \tau, \chi_{i}\right)\left\{\left\|\Lambda^{s-3 / 2}(\lambda) \chi_{1}^{0} h\right\|+\left\|\Lambda^{\tau}(\lambda) h\right\|\right\}
$$

where $c_{0}$ does not depend on $\lambda, s, \tau$ and $\chi_{i}$.

Proof. It is sufficient in order to show 1) that we prove the same estimate for $e^{-\Lambda(\lambda) y} \cdot \chi_{2}$ without assumption for $\chi_{2}$ to bc non-negativc. 


$$
\begin{aligned}
& \left\|\Lambda^{s}(\lambda) e^{-\Lambda(\lambda){ }^{y}} \chi_{2} h\right\|_{+}^{2} \\
& =\int_{0}^{\infty}\left\||\zeta|^{s} e^{-|\zeta| y_{\chi_{2} h}}\right\|^{2} d y, \quad \zeta=(\xi, \lambda) \\
& =\left.\int d \xi \int_{0}^{\infty}|\zeta|^{2 s} e^{-|\zeta| y} \widehat{\mid \chi_{2} h}\right|^{2} d y \\
& =\left.\sum_{v=1}^{l} \int|\zeta|^{2 s-v} \partial_{y}^{v-1}\left|\widehat{\chi_{2} h}\right|^{2}\right|_{y=0} d \xi \\
& +\int d \xi \int_{0}^{\infty}|\zeta|^{2 s-l} e^{-|\zeta| y} \partial_{y}^{l}\left|\widehat{\chi_{2} h}\right|^{2} d y .
\end{aligned}
$$

We use many times this equality and Lemma 3.14. And we modify $\left.\partial_{y}^{v} \chi_{2}\right|_{y=0}$ by $\chi_{1}^{0}$ except for the principal term because $\left.\chi_{1}^{0} \partial_{y}^{v} \chi_{2}\right|_{y=0}=\left.\partial_{y}^{v} \chi_{2}\right|_{y=0}$. The last terms form the rcmained term $\left.\left\|\Lambda^{\tau}(\lambda) h\right\| .2\right)$, 3) and 4) are proved similarly.

End of proof.

\section{§4. Existence, Uniqueness and Regularity (Special Case)}

In this section we shall treat a special case, that is, the domain is $R_{+}^{n+1} \equiv\left\{(x, y) ; x \in R^{n}, y \in R^{1}\right.$ and $\left.y>0\right\}$ and the equation is

$$
\left\{\begin{array}{l}
\left\{\frac{\partial}{\partial y}+M\left(x, \partial_{x}, \lambda\right)\right\} u=f \\
\left.B\left(x, \partial_{x}, \lambda\right) u\right|_{y-0}=y
\end{array},\right.
$$

where $M\left(x, \partial_{x}, \lambda\right)$ and $B\left(x, \partial_{\lambda}, \lambda\right)$ are pseudo-differcntial operators whose symbols are $M(x, \xi, \lambda)$ and $B(x, \xi, \lambda)$ in the section 2 and satisfy the assumption (A). We shall show existence, uniqueness and regularity of solutions for (4.1) by means of constructing right and left parametrices with the pseudo-differential operators which we have dealt with in the sections 2 and 3 .

We first make (4.1) more exact. We assume that $u \in H_{m}^{0}\left(R_{+}^{n+1}\right)$, $f \in H_{m}^{0}\left(R_{+}^{n+1}\right)$ and

$$
\left\{\frac{\partial}{\partial y}+M\left(x, \partial_{x}, \lambda\right)\right\} u(x, y)=f(x, y) ; y>0
$$


Lemma 4.1. $u(x, y)$ can be considered as $H_{m}^{-1 / 2}\left(R^{n}\right)$ valued continuous function in $y$ on $[0, \infty)$. Therefore, the boundary value of $u(x, y)$ in $R_{+}^{n+1}$ which belongs in $H_{m}^{-1 / 2}\left(R^{n}\right)$ is well-defined.

Proof. We first prove the following inequality (4.3) in the case that $u \in C_{0}^{\infty}\left(\overline{R_{+}^{n+1}}\right)$. To (4.2) we operate $\Lambda^{-1}(\lambda)$ which is a pseudo-differential operator with the symbol $\left(|\xi|^{2}+\lambda^{2}\right)^{-1 / 2}$ and consider the inner product of it and $u(\cdot, y)$ in $x$.

$$
\begin{aligned}
-\frac{\partial}{\partial y}< & \Lambda^{-1}(\lambda) u(\cdot, y), u(\cdot, y)> \\
= & 2 \operatorname{Re}\left\{<\Lambda^{-1}(\lambda) \cdot M\left(x, \partial_{x}, \lambda\right) u(\cdot, y), u(\cdot, y)>\right. \\
& \left.-<\Lambda^{-1}(\lambda) f(\cdot, y), u(\cdot, y)>\right\} .
\end{aligned}
$$

Integrating from 0 to $\infty$ in $y$, we obtain that

$$
\begin{aligned}
& <\Lambda^{-1}(\lambda) u(\cdot, 0), u(\cdot, 0)> \\
& \quad=2 \operatorname{Re}\left\{\left(\Lambda^{-1}(\lambda) M\left(x, \partial_{x}, \lambda\right) u, u\right)_{+}-\left(\Lambda^{-1}(\lambda) f, u\right)_{+}\right\}
\end{aligned}
$$

where $<,>$ and $(,)_{+}$are the standard inner products in $H_{m}^{0}\left(R^{n}\right)$ and $H_{m}^{0}\left(R_{+}^{n+1}\right)$, respectively. $\Lambda^{-1}(\lambda) \cdot M\left(x, \partial_{x}, \lambda\right)$ is a bounded operator on $H_{m}^{0}\left(R^{n}\right)$, also on $H_{m}^{0}\left(R_{+}^{n+1}\right)$. Thus, we obtain that

$$
\begin{aligned}
& \ll \Lambda^{-1 / 2}(\lambda) u(\cdot, 0) \gg^{2} \\
& =<\Lambda^{-1}(\lambda) u(\cdot, 0), u(\cdot, 0)> \\
& \leqq c\left\{\|u\|_{+}^{2}+\|f\|_{+}\|u\|_{+}\right\},
\end{aligned}
$$

where the constant $c$ does not depend on $\lambda$. Let us prove it in the case that, $u, f \in H_{m}^{0}\left(R_{+}^{n+1}\right)$ satisfy (4.2). Since $\left\{\frac{\partial}{\partial y}+M\left(x, \partial_{x}, \lambda\right)\right\}$ is an elliptic system, $u(x, y+a) \in H_{m}^{1}\left(R_{+}^{n+1}\right)$ for $a>0$. This means that $u(\cdot, y)$ is an $H_{m}^{1 / 2}\left(R^{n}\right)$, also $H_{m}^{-1 / 2}\left(R^{n}\right)$ valued continuous function in $y$ on $(0$, $\infty)$. Since $C_{0}^{\infty}\left(\overline{R_{+}^{n+1}}\right)$ is dense in $H_{m}^{1}\left(\overline{R_{+}^{n+1}}\right), u(x, y+a)-u(x, y+b)$ satisfies (4.3) for $a, b>0$. Since $\lim _{a \rightarrow 0} u(x, y+a)=u(x, y)$ and $\lim _{a \rightarrow 0} f(x, y+a)=f(x, y)$ in $H_{m}^{0}\left(R_{+}^{n+1}\right)$, there exists the limit of $u(\cdot, a)$ in $\stackrel{a}{H}_{m}^{-1 / 2}\left(R^{n}\right)$ as $a \rightarrow+0$. Putting $u(\cdot, 0) \equiv \lim _{a \rightarrow 0} u(\cdot, a), u(\cdot, 0)$ and $u$ satisfy (4.3). End of proof. 
Definition 4.1. Let us define $W$ as $u \in W$ if and only if $u$ and $\left\{\frac{\partial}{\partial y}+M\left(x, \partial_{x}, \lambda\right)\right\} u \in H_{m}^{0}\left(R_{+}^{n+1}\right)$.

Remark. $W$ does not depend on $\lambda$ because any homogeneous function $p(\xi, \lambda)$ of order $\alpha$ in $(\xi, \lambda)$ is written as $p(\xi, \lambda)=p(\xi, 0)+\lambda q(\xi, \lambda)$, where $q(\xi, \lambda)$ is a homogeneous function of order $\alpha-1$ in $(\xi, \lambda)$.

Corollary of Lemma 4. 且. $C_{0}^{\infty}\left(\overline{R_{+}^{n+1}}\right)$ is dense in $W$ with the norm $\|u\| \equiv\left(\|u\|_{+}^{2}+\left\|\left\{\frac{\partial}{\partial y}+M\left(x, \partial_{x}, \lambda\right)\right\} u\right\|_{+}^{2}\right)^{1 / 2}$.

Definition 4.2. Let $u s$ define $W_{b}$ as $u \in W_{b}$ if and only if $u \in W$ and $B\left(x, \partial_{x}, \lambda\right) u(\cdot, 0) \in H_{l}^{1 / 2}\left(R^{n}\right)$.

Remark. $W_{b}$ does not depend on $\lambda$ because of the same reason as the remark of Definition 4.1.

Remark. When we regard as $W\left(W_{b}\right) \subset H_{m}^{0}\left(R_{+}^{n+1}\right) \times H_{m}^{-1 / 2}\left(R^{n}\right), W\left(W_{b}\right)$ stand for the elements $(f, g)$ such that $f \in W\left(W_{b}\right)$ and $g=\left.f\right|_{y=0}$.

Definition 4.3. Let $C_{0}(\lambda)$ stand for the closed operator, from $H_{m}^{0}\left(R_{+}^{n+1}\right) \times H_{m}^{-1 / 2}\left(R^{n}\right)$ to $H_{m}^{0}\left(R_{+}^{n+1}\right) \times H_{l}^{1 / 2}\left(R^{n}\right)$,

$\left(\begin{array}{cc}\frac{\partial}{\partial y}+M\left(x, \partial_{x}, \lambda\right), & 0 \\ 0 & , B\left(x, \partial_{x}, \lambda\right)\end{array}\right)$ whose definition domain is $W_{b}$.

\section{$\S \S 4.1$. Existence of Solutions}

We first define some operators in the following way.

Definition 4.4. $\quad A_{0}(\lambda) \equiv \frac{\partial}{\partial y}+M\left(x, \partial_{x}, \lambda\right)$

$$
A(x, \xi, \eta, \lambda) \equiv i \eta+M(x, \xi, \lambda)
$$

Definition 4.5 .

$$
S_{1}(\lambda) f \equiv \iint e^{i(x \xi+y \eta)} A(x, \xi, \eta, \lambda)^{-1} \hat{f}(\xi, \eta) d \xi d \eta \quad \in H_{m}^{1}\left(R_{+}^{n+1}\right)
$$




$$
\begin{aligned}
& S_{2}(\lambda) f \equiv \iint e^{i x \xi} A(x, \xi, \eta, \lambda)^{-1} \hat{f}(\xi, \eta) d \xi d \eta \quad \in H_{m}^{1 / 2}\left(R^{n}\right) \\
& S_{3}(\lambda) g \equiv \int e^{i x \xi} e^{-M(x, \xi, \lambda) y} P_{-}(x, \xi, \lambda) \hat{g}(\xi) d \xi \quad \in H_{m}^{0}\left(R_{+}^{n+1}\right) \\
& S_{4}(\lambda) g \equiv \int e^{i x \xi} P_{-}(x, \xi, \lambda) \hat{g}(\xi) d \xi \quad \in H_{m}^{-1 / 2}\left(R^{n}\right) \\
& S_{5}(\lambda) h \equiv \int e^{i x \xi} E(x, \xi, \lambda) \hat{h}(\xi) d \xi \quad \in H_{m}^{-1 / 2}\left(R^{n}\right)
\end{aligned}
$$

where $\quad f \in H_{m}^{0}\left(R_{+}^{n+1}\right), \quad g \in H_{m}^{-1 / 2}\left(R^{n}\right), \quad h \in H_{l}^{1 / 2}\left(R^{n}\right)$,

$$
\begin{aligned}
& \hat{f}(\xi, \eta) \equiv \iint_{R_{+}^{n+1}} e^{-i(x \xi+y \eta)} f(x, y) d x d y, \\
& \hat{g}(\check{\zeta}) \equiv \int e^{-i x \xi} g(x) d x \quad \text { and } \\
& \hat{h}(\xi) \equiv \int e^{-i x \xi} h(x) d x .
\end{aligned}
$$

(We use the symbol of integral for Fourier transform in $\xi,(\xi, \eta), x$ or $(x, y)$ to be visible though they may not be integrable. Refer to the section 2 for definitions of $e^{-M y} P_{-}, P_{-}$and $E$.) These operators are well defined by Lemmas 3.10 and 3.12. $\left(S_{1}(\lambda) f\right)(\cdot, y)$ is an $H_{m}^{1 / 2}\left(R^{n}\right)$ valued continuous function in $y$ and $\left(S_{1}(\lambda) f\right)(\cdot, 0)=S_{2}(\lambda) f . \quad\left(S_{3}(\lambda) g\right)(\cdot, y)$ is an $H_{m}^{-1 / 2}\left(R^{n}\right)$ valued continuous function in $y \geqq 0$ and $\left(S_{3}(\lambda) g\right)(\cdot, 0)$ $=S_{4}(\lambda) g$.

Definition 4.6. Let us put

$$
\begin{aligned}
& B_{0}(\lambda) \equiv B\left(x, \partial_{x}, \lambda\right) \\
& S(\lambda) \equiv\left(\begin{array}{ll}
S_{1}(\lambda), & S_{3}(\lambda) \cdot S_{5}(\lambda) \\
S_{2}(\lambda), & S_{4}(\lambda) \cdot S_{5}(\lambda)
\end{array}\right) .
\end{aligned}
$$

$S(\lambda)$ is a continuous linear operator from $H_{m}^{0}\left(R_{+}^{n+1}\right) \times H_{l}^{1 / 2}\left(R^{n}\right)$ to $H_{m}^{0}\left(R_{+}^{n+1}\right) \times H_{m}^{-1 / 2}\left(R^{n}\right)$ with a parameter $\lambda$. We operate $\left(\begin{array}{cc}A_{0}(\lambda), & 0 \\ 0, & B_{0}(\lambda)\end{array}\right)$ to $S(\lambda)$ from the left hand side and represent it by pseudo-differential 
operators.

$$
\begin{aligned}
& \left(\begin{array}{cc}
A_{0}(\lambda), & 0 \\
0, & B_{0}(\lambda)
\end{array}\right) \cdot S(\lambda)=\left(\begin{array}{cc}
A_{0}(\lambda) \cdot S_{1}(\lambda), & A_{0}(\lambda) \cdot S_{3}(\lambda) \cdot S_{5}(\lambda) \\
B_{0}(\lambda) \cdot S_{2}(\lambda), & B_{0}(\lambda) \cdot S_{4}(\lambda) \cdot S_{5}(\lambda)
\end{array}\right) \\
& =I+T(\lambda) \\
& T(\lambda)=\left(\begin{array}{cc}
T_{11}(\lambda), & T_{12}(\lambda) \\
T_{21}(\lambda), & T_{22}(\lambda)
\end{array}\right) \\
& T_{11}(\lambda)=\left[M\left(x, \partial_{x}, \lambda\right), S_{1}(\lambda)\right]
\end{aligned}
$$

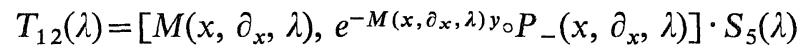

$$
\begin{aligned}
& T_{21}(\lambda)=B\left(x, \partial_{x}, \lambda\right) \cdot S_{2}(\lambda) \\
& T_{22}(\lambda)=\left[B\left(x, \partial_{x}, \lambda\right), P_{-}\left(x, \partial_{x}, \lambda\right)\right] \cdot S_{5}(\lambda)+\left[D\left(x, \partial_{x}, \lambda\right), S_{5}(\lambda)\right] .
\end{aligned}
$$

([p, $q]=p \cdot q-p \circ q$, where $p$ and $q$ are pseudo-differential operators and $\circ$ is product in symbol space.)

\section{Lemma 4.2.}

1) $\left\|T_{11}(\lambda)\right\|<c \lambda^{-1}$ on $H_{m}^{0}\left(R_{+}^{n+1}\right)$

2) $\left\|T_{12}(\lambda)\right\|<c \lambda^{-1}$ from $H_{l}^{1 / 2}\left(R^{n}\right)$ to $H_{m}^{0}\left(R_{+}^{n+1}\right)$

3) $\left\|T_{21}(\lambda)\right\|<c$ from $H_{m}^{0}\left(R_{+}^{n}\right)$ to $H_{l}^{1 / 2}\left(R^{n}\right)$

4) $\left\|T_{22}(\lambda)\right\|<c \lambda^{-1}$ on $H_{l}^{1 / 2}\left(R^{n}\right)$

5) $\|T(\lambda) \cdot T(\lambda)\|<c \lambda^{-1}$ on $H_{m}^{0}\left(R_{+}^{n+1}\right) \times H_{l}^{1 / 2}\left(R^{n}\right)$

6) There exists the inverse operator of $I+T(\lambda)$ on $H_{m}^{0}\left(R_{+}^{n+1}\right) \times H_{l}^{1 / 2}\left(R^{n}\right)$ for sufficiently large $\lambda$.

$$
(I+T(\lambda))^{-1}=(I-T(\lambda))(I-T(\lambda) \cdot T(\lambda))^{-1}
$$

and $\left\|(I+T(\lambda))^{-1}\right\|<c ; \lambda \geqq{ }^{\exists} \lambda_{0}$, where we use $\left\|\Lambda^{1 / 2}(\lambda) \varphi\right\|, \varphi \in H_{l}^{1 / 2}\left(R^{n}\right)$ as the norms of $H_{l}^{1 / 2}\left(R^{n}\right)$ with parameter $\lambda$. 
Proof. If we assume 1), 2), 3) and 4), then 5), also 6) are clear. From the definitions, $T_{i j}$ are combinations of pseudo-differential operators which we have dealt with in Lemmas 3.10 and 3.12. We can use them to obtain 1), 2), 3) and 4). For example, by 5), 13) and 14) of Lemma 3.10 ,

$$
\begin{aligned}
&\left\|\Lambda^{1 / 2}(\lambda) T_{22}(\lambda) h\right\| \\
& \leqq\left\|\Lambda^{1 / 2}(\lambda) \cdot\left[B\left(x, \partial_{x}, \lambda\right), P_{-}\left(x, \partial_{x}, \lambda\right)\right] \cdot E\left(x, \partial_{x}, \lambda\right) h\right\| \\
&+\left\|\Lambda^{1 / 2}(\lambda) \cdot\left[D\left(x, \partial_{x}, \lambda\right), E\left(x, \partial_{x}, \lambda\right)\right] h\right\| \\
& \leqq c\left\|\Lambda^{-1 / 2}(\lambda) \cdot E\left(x, \partial_{x}, \lambda\right) h\right\| \\
&+\left\|\Lambda^{1 / 2}(\lambda) \cdot\left[D\left(x, \partial_{x}, \lambda\right), E\left(x, \partial_{x}, \lambda\right)\right] h\right\| \\
& \leqq c \lambda^{-1}\left\|\Lambda^{1 / 2}(\lambda) h\right\| .
\end{aligned}
$$

End of proof.

Theorem 2. There exist bounded operators $R_{0}(\lambda)$ from $H_{m}^{0}\left(R_{+}^{n+1}\right) \times$ $H_{l}^{1 / 2}\left(R^{n}\right)$ to $H_{m}^{0}\left(R_{+}^{n+1}\right) \times H_{m}^{-1 / 2}\left(R^{n}\right)$ for $\lambda \geqq \mathrm{E} \lambda_{0}$ such that range of $R_{0}(\lambda)$ $\subset W_{b}, C_{0}(\lambda) \cdot R_{0}(\lambda)=I$ on $H_{m}^{0}\left(R_{+}^{n+1}\right) \times H_{l}^{1 / 2}\left(R^{n}\right)$ and $\left\|R_{0}(\lambda)\right\| \leqq c \lambda^{-1}$.

Proof. We can define $R_{0}(\lambda) \equiv S(\lambda) \cdot(I+T(\lambda))^{-1}$ by Lemma 4.2. Then $\left(\begin{array}{cc}A_{0}(\lambda), & 0 \\ 0 & B_{0}(\lambda)\end{array}\right) \cdot R_{0}(\lambda)=I$ by (4.4). The remark after the definition 4.4 for $S(\lambda)$ shows that range of $R_{0}(\lambda) \subset W_{b}$. Thus

$$
C_{0}(\lambda) \cdot R_{0}(\lambda)=I
$$

The estimate for $R_{0}(\lambda)$ follows from the estimates for $S_{1}(\lambda) \sim S_{5}(\lambda)$ of Lemmas 3.10 and 3.12. Here, we have to notice that we use the norms with parameter $\lambda$ such that $\left\|\Lambda^{s}(\lambda) \varphi\right\|, \varphi \in H_{m}^{s}\left(R^{n}\right)$ for $H_{m}^{s}\left(R^{n}\right)$.

End of proof.

\section{$\S \S 4.2$. Uniqueness and regularity of solutions}

Definition 4.7. $S_{6}(\lambda) f \equiv \iint e^{i(x \xi+y \eta)} A(x, \xi, \eta, \lambda)^{-1} i \eta \hat{f}(\xi, \eta) d \xi d \eta$ $\in H_{m}^{0}\left(R_{+}^{n+1}\right)$, where $f \in H_{m}^{0}\left(R_{+}^{n+1}\right)$ and 


$$
\hat{f}(\xi, \eta) \equiv \iint_{R_{+}^{n+1}} e^{-i(x \xi+y \eta)} f(x, y) d x d y .
$$

Lemma 4.3. Let $\varphi \in C_{0}^{\infty}\left(\overline{R_{+}^{n+1}}\right)$.

1) $S_{1}(\lambda) \frac{\partial}{\partial y} \varphi=S_{6}(\lambda)+S_{3}(\lambda) \varphi(\cdot, 0)$ in $R_{+}^{n+1}$.

2) $S_{1}(\lambda) \cdot A_{0}(\lambda) \varphi=\varphi+S_{3}(\lambda) \varphi(\cdot, 0)+\left[S_{1}(\lambda), M\left(x, \partial_{x}, \lambda\right)\right] \varphi$, in $R_{+}^{n+1}$.

(We omit the proof.)

Lemma 4.4. Let $u \in W$ and $f=A_{0}(\lambda) u$.

1) $u+S_{3}(\lambda) u(\cdot, 0)+\left[S_{1}(\lambda), M\left(x, \partial_{x}, \lambda\right)\right] u=S_{1}(\lambda) f$.

2) $u(\cdot, 0)+S_{4}(\lambda) u(\cdot, 0)+\left.\left[S_{1}(\lambda), M\left(x, \partial_{x}, \lambda\right)\right] u\right|_{y=0}=S_{2}(\lambda) f$.

Proof. If $\left.u \in C_{0}^{\infty}\left(\overline{R_{+}^{n+1}}\right), 1\right)$ and 2) are valid by 2) of Lemma 4.3. $\left[S_{1}(\lambda), M\left(x, \partial_{x}, \lambda\right)\right]$ is a bounded operator from $H_{m}^{0}\left(R_{+}^{n+1}\right)$ to $H_{m}^{1}\left(R_{+}^{n+1}\right)$, $S_{3}(\lambda)$ from $H_{m}^{-1 / 2}\left(R^{n}\right)$ to $H_{m}^{0}\left(R_{+}^{n+1}\right), S_{1}(\lambda)$ from $H_{m}^{0}\left(R_{+}^{n+1}\right)$ to $H_{m}^{1}\left(R_{+}^{n+1}\right)$, $S_{4}(\lambda)$ from $H_{m}^{-1 / 2}\left(R^{n}\right)$ to $H_{m}^{-1 / 2}\left(R^{n}\right)$ and $S_{2}(\lambda)$ from $H_{m}^{0}\left(R_{+}^{n+1}\right)$ to $H_{m}^{1 / 2}\left(R^{n}\right)$. $C_{0}^{\infty}\left(\overline{R_{+}^{n+1}}\right)$ is dense in $W$ with the norm $\left(\|u\|_{+}^{2}+\|f\|_{+}^{2}\right)^{1 / 2}$ by Corollary of Lemma 4.1. Thus, 1) and 2) are valid for $u \in W$. End of proof.

Definition 4.8. For $u \in H_{m}^{0}\left(R_{+}^{n+1}\right)$ and $v \in H_{m}^{-1 / 2}\left(R^{n}\right)$, we define $S_{7}(\lambda)$, $S_{8}(\lambda)$ and $S_{9}(\lambda)$ as

$$
\begin{array}{rlrl}
S_{7}(\lambda) u \equiv\left[S_{1}(\lambda), M\left(x, \partial_{x}, \lambda\right)\right] u & & \in H_{m}^{1}\left(R_{+}^{n+1}\right) \\
\left.S_{8}(\lambda) u \equiv\left[S_{1}(\lambda), M\left(x, \partial_{x}, \lambda\right)\right] u\right|_{y=0} & \in H_{m}^{1 / 2}\left(R^{n}\right) \\
S_{9}(\lambda) v \equiv\left\{S_{5}(\lambda) \cdot\left[B_{0}(\lambda), S_{4}(\lambda)\right]+\left[S_{5}(\lambda), D\left(x, \partial_{x}, \lambda\right)\right]\right\} v \\
& \in H_{m}^{-1 / 2}\left(R^{n}\right) .
\end{array}
$$

Lemma 4.5. Let $u \in W_{b}, f=A_{0}(\lambda) u$ and $g=B_{0}(\lambda) u(\cdot, 0)$.

$$
\begin{aligned}
& u(\cdot, 0)-S_{9}(\lambda) u(\cdot, 0)+S_{8}(\lambda) u-S_{2}(\lambda) \cdot B_{0}(\lambda) \cdot S_{8}(\lambda) u \\
& \quad=S_{5}(\lambda) g+S_{5}(\lambda) \cdot B_{0}(\lambda) \cdot S_{5}(\lambda) f \text { in } H_{m}^{-1 / 2}\left(R^{n}\right) .
\end{aligned}
$$


Proof. We operate $B_{0}(\lambda)$ to the equality 2) of Lemma 4.4 from the left hand side.

$$
\begin{aligned}
& B_{0}(\lambda) u(\cdot, 0)+B_{0}(\lambda) \cdot S_{4}(\lambda) u(\cdot, 0)+B_{0}(\lambda) \cdot S_{8}(\lambda) u \\
& \quad=B_{0}(\lambda) \cdot S_{2}(\lambda) f .
\end{aligned}
$$

We further operate $S_{5}(\lambda)$ to this equality.

$$
\begin{aligned}
& S_{5}(\lambda) g+S_{5}(\lambda) \cdot B_{0}(\lambda) \cdot S_{4}(\lambda) u(\cdot, 0)+S_{5}(\lambda) \cdot B_{0}(\lambda) \cdot S_{8}(\lambda) u \\
& \quad=S_{5}(\lambda) \cdot B_{0}(\lambda) \cdot S_{2}(\lambda) f .
\end{aligned}
$$

Since

$$
\begin{aligned}
& S_{5}(\lambda) \cdot B_{0}(\lambda) \cdot S_{4}(\lambda) \\
& \quad=S_{4}(\lambda)+S_{5}(\lambda) \cdot\left[B_{0}(\lambda), S_{4}(\lambda)\right]+\left[S_{5}(\lambda), D\left(x, \partial_{x}, \lambda\right)\right], \\
& S_{4}(\lambda) u(\cdot, 0)=-\left\{S_{5}(\lambda) \cdot\left[B_{0}(\lambda), S_{4}(\lambda)\right]+\left[S_{5}(\lambda), D\left(x, \partial_{x}, \lambda\right)\right]\right\} u(\cdot, 0) \\
& \quad-S_{5}(\lambda) \cdot B_{0}(\lambda) \cdot S_{8}(\lambda) u-S_{5}(\lambda) g+S_{5}(\lambda) \cdot B_{0}(\lambda) \cdot S_{2}(\lambda) f .
\end{aligned}
$$

Putting this in the equality 2) of Lemma 4.4 again, we obtain the equality of this Lemma 4.5 .

End of proof.

\section{Definition 4.9.}

$$
\begin{aligned}
& Z_{11}(\lambda) \equiv S_{7}(\lambda), \quad Z_{12}(\lambda) \equiv S_{3}(\lambda) \\
& Z_{21}(\lambda) \equiv S_{8}(\lambda)-S_{5}(\lambda) \cdot B_{0}(\lambda) \cdot S_{8}(\lambda), \quad Z_{22}(\lambda) \equiv-S_{9}(\lambda) \\
& Z(\lambda) \equiv\left(\begin{array}{c}
Z_{11}(\lambda), Z_{12}(\lambda) \\
Z_{21}(\lambda), Z_{22}(\lambda)
\end{array}\right), \tilde{S}(\lambda) \equiv\left(\begin{array}{cc}
S_{1}(\lambda), & 0 \\
S_{5}(\lambda) \cdot B_{0}(\lambda) \cdot S_{2}(\lambda), & S_{5}(\lambda)
\end{array}\right) .
\end{aligned}
$$

Lemma 4.6. Let $u \in W_{b}, f=A_{0}(\lambda) u$ and $g=B_{0}(\lambda) u(\cdot, 0)$.

$$
\{I+Z(\lambda)\}\left(\begin{array}{l}
u \\
u(\cdot, 0)
\end{array}\right)=\tilde{S}(\lambda)\left(\begin{array}{l}
f \\
g
\end{array}\right)
$$

Proof. It follows from the definition of $Z(\lambda), 1)$ of Lemma 4.4 and Lemma 4.5.

End of proof. 
Lemma 4.7. Let $\lambda \geqq 1$.

1) $\left\|Z_{11}(\lambda)\right\|<c \lambda^{-1}$

2) $\left\|Z_{12}(\lambda)\right\|<c$

3) $\left\|Z_{21}(\lambda)\right\|<c \lambda^{-1}$

4) $\left\|Z_{22}(\lambda)\right\|<c \lambda^{-1}$

5) $\|Z(\lambda) \cdot Z(\lambda)\|<c \lambda^{-1}$

6) There exists the inverse operator of $I+Z(\lambda)$ on $H_{m}^{0}\left(R_{+}^{n+1}\right) \times H_{m}^{-1 / 2}\left(R^{n}\right)$ for sufficiently large $\lambda$.

$$
\begin{aligned}
& (I+Z(\lambda))^{-1}=(I-Z(\lambda))(I-Z(\lambda) \cdot Z(\lambda))^{-1} \\
& \left\|(I+Z(\lambda))^{-1}\right\|<c ; \lambda \geqq^{\exists} \lambda_{0},
\end{aligned}
$$

where we use $\left\|\Lambda^{-1 / 2}(\lambda) \varphi\right\|, \varphi \in H_{m}^{-1 / 2}\left(R^{n}\right)$ as the norms of $H_{m}^{-1 / 2}\left(R^{n}\right)$ with parameter $\lambda$.

Proof. 6) follows from 5), which follows from 1), 2), 3) and 4), which follow from Lemmas 3.10 and 3.12. For example, by 5), 13) and 15) of Lemma 3.10 ,

$$
\begin{aligned}
& \left\|\Lambda^{-1 / 2}(\lambda) \cdot Z_{22}(\lambda) h\right\| \\
& \leqq \\
& \quad\left\|\Lambda^{-1 / 2}(\lambda) \cdot E\left(x, \partial_{x}, \lambda\right) \cdot\left[B\left(x, \partial_{x}, \lambda\right), P_{-}\left(x, \partial_{x}, \lambda\right)\right] h\right\| \\
& \quad+\left\|\Lambda^{-1 / 2}(\lambda) \cdot\left[E\left(x, \partial_{x}, \lambda\right), D\left(x, \partial_{x}, \lambda\right)\right] h\right\| \\
& \leqq c \lambda^{-1}\left\|\Lambda^{-1 / 2}(\lambda) h\right\| .
\end{aligned}
$$

End of proof.

Theorem 3. For any $\lambda \geqq \lambda_{0}$, which is a fixed constant, there exists a bounded operator $L_{0}(\lambda)$, from $H_{m}^{0}\left(R_{+}^{n+1}\right) \times H_{l}^{1 / 2}\left(R^{n}\right)$ to $H_{m}^{0}\left(R_{+}^{n+1}\right) \times$ $H_{m}^{-1 / 2}\left(R^{n}\right)$, which is a left inverse operator of $C_{0}(\lambda)$, that is, $\left(\begin{array}{c}A_{0}(\lambda) \text {, } \\ 0\end{array}\right.$, $\left.\begin{array}{c}0 \\ B_{0}(\lambda)\end{array}\right)$ whose definition domain is $W_{b} \subset H_{m}^{0}\left(R_{+}^{n+1}\right) \times H_{m}^{-1 / 2}\left(R^{n}\right)$ and satisfies the estimate

$$
\left\|L_{0}(\lambda)\right\|<c \lambda^{-1}
$$


Proof. We consider the operator $(I+Z(\lambda))^{-1} \tilde{S}(\lambda)$. This is what we need because of Lemma 4.6. The estimate is obtained by combining those of $(I+Z(\lambda))^{-1}, S_{1}(\lambda), S_{5}(\lambda)$ and $S_{2}(\lambda)$. (See Lemmas 3.10 and 3.12.)

End of proof.

Theorem 4. Let $u \in H_{m}^{0}\left(R_{+}^{n+1}\right)$. If $u$ satisfies that $A_{0}(\lambda) u=0$ and $B_{0}(\lambda) u(\cdot, 0)=0$ for a sufficiently large $\lambda$, then $u$ is zero of $H_{m}^{0}\left(R_{+}^{n+1}\right)$.

Proof. This is a corollary of Theorem 3. End of proof.

We now go on to the next problem, the regularity of solutions. We show the regularity in $x$ of solutions because the regularity in $y$ is brought by the equation.

Lemma 4.8. Let $u \in C_{0}^{\infty}\left(\overline{R_{+}^{n+1}}\right), v \in C_{0}^{\infty}\left(R^{n}\right), \chi_{i} \in C^{\infty}\left(\overline{R_{+}^{n+1}}\right)$ and $\chi_{i}^{0}(x) \equiv$ $\chi_{i}(x, 0) \in C^{\infty}\left(R^{n}\right)$ such that $\chi_{i} \equiv 1$ or $\in C_{0}^{\infty}\left(R_{+}^{n+1}\right), 0 \leqq \chi_{i} \leqq 1$ and $\chi_{1} \chi_{2}=\chi_{2}$. Then, there is a positive constant $c_{0}$ not depending on $\lambda \in[1, \infty), s \geqq 0$ and $\chi_{i}$ such that

1) $\left\|\Lambda^{s}(\lambda) \chi_{2} Z_{11}(\lambda) u\right\|_{+} \leqq c_{0} \lambda^{-1}\left\|\Lambda^{s}(\lambda) \chi_{2} u\right\|_{+}$

$$
+c\left(s, \chi_{i}\right) \lambda^{-3 / 2}\left\{\left\|\Lambda^{s-1 / 2}(\lambda) \chi_{1} u\right\|_{+}+\|u\|_{+}\right\}
$$

2) $\left\|\Lambda^{s}(\lambda) \chi_{2} Z_{12}(\lambda) v\right\|_{+} \leqq c_{0}\left\|\Lambda^{s-1 / 2}(\lambda) \chi_{2}^{0} v\right\|$

$$
+c\left(s, \chi_{i}\right) \lambda^{-1 / 2}\left\{\left\|\Lambda^{s-1}(\lambda) \chi_{1}^{0} v\right\|+\left\|\Lambda^{-1 / 2}(\lambda) v\right\|\right\}
$$

3) $\left\|\Lambda^{s-1 / 2}(\lambda) \chi_{2}^{0} Z_{21}(\lambda) u\right\| \leqq c_{0} \lambda^{-1}\left\|\Lambda^{s}(\lambda) \chi_{2} u\right\|_{+}$

$$
+c\left(s, \chi_{i}\right) \lambda^{-3 / 2}\left\{\left\|\Lambda^{s-1 / 2}(\lambda) \chi_{1} u\right\|_{+}+\|u\|_{+}\right\}
$$

4) $\left\|\Lambda^{s-1 / 2}(\lambda) \chi_{2}^{0} Z_{22}(\lambda) v\right\| \leqq c_{0} \lambda^{-1}\left\|\Lambda^{s-1 / 2}(\lambda) \chi_{2}^{0} v\right\|$

$$
+c\left(s, \chi_{i}\right) \lambda^{-3 / 2}\left\{\left\|\Lambda^{s-1}(\lambda) \chi_{1}^{0} v\right\|+\left\|\Lambda^{-1 / 2}(\lambda) v\right\|\right\},
$$

where $\|\cdot\|_{+}$and $\|\cdot\|$ are $L^{2}$-norms in $R_{+}^{n+1}$ and in $R^{n}$, respectively.

Proof. These follow from Lemmas 3.15 and 3.18. End of proof.

Lemma 4.9. Let $X$ be a Banach space and $\left\{p_{i}\right\}_{i=0, \ldots, l}$ be seminorms on $X$ such that $q_{l}$ is a norm of $X$, where $q_{j} \equiv\left(\sum_{i=0}^{j} p_{i}^{2}\right)^{1 / 2}$; 
$j=0, \ldots, l$. Let $T$ be a bounded linear operator on $X$ such that

$$
p_{i}(T x)^{2} \leqq a_{i} p_{i}(x)^{2}+b_{i} q_{i-1}(x)^{2} ; \quad i=0, \ldots, l,
$$

where $0 \leqq a_{i}<a_{i+1}<1$ and $q_{-1}=0$. Then, $I+T$ is an isomorphism on $X$ and it satisfies the estimate:

$$
q_{l}\left((I+T)^{-1} x\right)^{2} \leqq\left(1-\sqrt{a_{l}}\right)^{-2} q_{l}(x)^{2}+c q_{l-1}(x)^{2} .
$$

Proof. We consider $\tilde{q} \equiv\left(\sum_{i=0}^{l} c_{i} p_{i}^{2}\right)^{1 / 2} ; c_{i}=1+d_{i} /\left(a_{l}-a_{i}\right), d_{i}=\sum_{j=i+1}^{l} b_{j} c_{j}$ $(i=0, \ldots, l-1)$ and $d_{l}=0$. Then, $\tilde{q}$ is a norm of $X$ and $\tilde{q}(T x)^{2} \leqq a_{l} \tilde{q}(x)^{2}$, that is, $T$ is a contraction operator on $X$ with the norm $\tilde{q}$. Thus, there exists $(I+T)^{-1}$, which satisfies the estimate:

$$
\tilde{q}\left((I+T)^{-1} x\right) \leqq\left(1-\sqrt{a_{l}}\right)^{-1} \tilde{q}(x) . \quad \text { End of proof. }
$$

Definition 4.10. Let $s \geqq 0$ and $i=0$ or 1 .

1) $Y_{i}^{s}(\lambda) \equiv L^{2}\left(R_{+}^{1} ; H_{m}^{s}\left(R^{n}\right)\right) \times H_{\alpha_{i}}^{s-1 / 2+i}\left(R^{n}\right)$ with the norm

$$
\|U\|_{Y_{i}^{s}(\lambda)} \equiv\left(\left\|\Lambda^{s}(\lambda) u\right\|_{+}^{2}+\left\|\Lambda^{s-1 / 2+i}(\lambda) v\right\|^{2}\right)^{1 / 2} ; U=(u, v) \in Y_{i}^{s}(\lambda),
$$

where $\alpha_{0}=m, \alpha_{1}=l$ and $L^{2}\left(R_{+}^{1} ; H_{m}^{s}\left(R^{n}\right)\right)$ is a space of $H_{m}^{s}\left(R^{n}\right)$-valued $L^{2}$-functions in $y \in(0, \infty)$.

2) Let $\chi=\left(\chi_{j}\right)_{j=0, \ldots, k}$ be a system of $C^{\infty}$-functions such that $\chi_{0} \equiv 1$, $\chi_{i} \in C^{\infty}\left(\overline{R_{+}^{n+1}}\right) ; \chi_{i} \equiv 1$ or $\in C_{0}^{\infty}\left(\overline{R_{+}^{n+1}}\right), 0 \leqq \chi_{i} \leqq 1$ and $\chi_{i+1} \chi_{i}=\chi_{i+1}(i \geqq 1)$.

$X_{i}^{s}(\lambda, \chi)$ stands for the closure of $C_{0}^{\infty}\left(\overline{R_{+}^{n+1}}\right) \times C_{0}^{\infty}\left(R^{n}\right)$ in the space $\left\{U ; \chi_{j} U \in Y_{i}^{\sigma_{j}}(\lambda), \sigma_{j}=s-(k-j) / 2,1 \leqq j \leqq k\right.$ and $\left.\sigma_{0}=0\right\}$ with the seminorms $p_{i j}(U) \equiv\left\|\chi_{j} U\right\|_{Y_{i}^{\sigma} j_{(\lambda)}}$, and the norm $\|U\|_{X_{j}^{s}(\lambda, \chi)} \equiv\left(\sum_{j=0}^{k} p_{i j}(U)^{2}\right)^{1 / 2}$, where $\chi_{j} U=\left(\chi_{j} u, \chi_{j}^{0} v\right) ; U=(u, v)$ and $\chi_{j}^{0}=\left.\chi_{j}\right|_{y=0}$.

Lemma 4.10. Let $0 \leqq s \leqq k / 2$. Then, for all $U \in X_{0}^{s}(\lambda, \chi)$,

$$
p_{0 j}(Z(\lambda) \cdot Z(\lambda) U) \leqq c_{0} \lambda^{-1} p_{0 j}(U)+c(s, \chi) \lambda^{-3 / 2} q_{0 j-1}(U)
$$

$0 \leqq j \leqq k$, where $q_{0 j} \equiv\left(\sum_{j=0}^{v} p_{0 v}^{2}\right)^{1 / 2} ; 0 \leqq j \leqq k, q_{0-1}=0$ and $c_{0}$ is a constant which does not depend on $s \geqq 0, \lambda \geqq 1$ and $\chi$.

Proof. These follow from Lemmas 4.7 and 4.8. End of proof. 
Lemma 4.11. There exist constants $\lambda_{0}$ and $c_{0}$, which do not depend on $s \geqq 0$ and $\chi$, such that for any $\lambda \geqq \lambda_{0}$ and $s \geqq 0, I+Z(\lambda)$ gives an isomorphism on $X_{0}^{s}(\lambda, \chi)$ and satisfies the following estimate.

$$
\left\|(I+Z(\lambda))^{-1} U\right\|_{X_{0}^{s}(\lambda, \chi)} \leqq c_{0}\|U\|_{X_{0}^{s}(\lambda, \chi)}+c(s, \chi) \lambda^{-1 / 2}\|U\|_{X_{0}^{s-1 / 2}\left(\lambda, \chi^{\prime}\right)},
$$

where $\chi^{\prime}=\left\{\chi_{j}\right\}_{0 \leqq j \leqq k-1}$ when $\chi=\left(\chi_{j}\right\}_{0 \leqq j \leqq k}$.

Proof. This follows from Lemmas 4.9 and 4.10 because $(I+Z(\lambda))^{-1}$ $=(I-Z(\lambda))(I-Z(\lambda) \cdot Z(\lambda))^{-1}$.

End of proof.

Lemma 4.12. Let $0 \leqq s \leqq k / 2$. Then, for all $U \in X_{1}^{s}(\lambda, \chi)$,

$$
p_{0 j}(\tilde{S}(\lambda) U) \leqq c_{0} \lambda^{-1} p_{1 j}(U)+c(s, \chi) \lambda^{-3 / 2} q_{1 j-1}(U) ; \quad 0 \leqq j \leqq k,
$$

where $q_{1 j} \equiv\left(\sum_{v=0}^{j} p_{1 v}^{2}\right)^{1 / 2} ; 0 \leqq j \leqq k, q_{1-1}=0$ and $c_{0}$ is a constant which does not depend on $s \geqq 0, \lambda \geqq 1$ and $\chi$. (See Definition 4.9 for $\tilde{S}(\lambda)$.)

Proof. These follow from Lemmas 3.10, 3.15 and 3.18. End of proof.

Theorem 5. The left inverse operator $L_{0}(\lambda)$ of $C_{0}(\lambda)$ in Theorem 3 is a bounded operator from $X_{1}^{s}(\lambda, \chi)$ to $X_{0}^{s}(\lambda, \chi)$ when $\lambda \geqq \lambda_{0}$ and $0 \leqq s$ $\leqq k / 2$, where $\lambda_{0}$ is in Lemma 4.11. It satisfies the following estimates for $U \in X_{1}^{s}(\lambda, \chi)$.

$$
p_{0 j}\left(L_{0}(\lambda) U\right) \leqq c_{0} \lambda^{-1} p_{1 j}(U)+c(s, \chi) \lambda^{-3 / 2} q_{1 j-1}(U)
$$

where $c_{0}$ is a constant not depending on $s \geqq 0, \lambda \geqq \lambda_{0}$ and $\chi$.

Proof. By the definition of $L_{0}(\lambda), L_{0}(\lambda)$ is written as $L_{0}(\lambda)=(I$ $+Z(\lambda))^{-1} \tilde{S}(\lambda)$. By Lemmas 4.11 and 4.12, $L_{0}(\lambda) U$ belongs to $X_{0}^{s}(\lambda, \chi)$ and satisfies the estimate in Theorem 5 when $U \in X_{1}^{s}(\lambda, \chi)$.

End of proof.

Corollary of Theorem 5. Let $u \in W_{b}, A_{0}(\lambda) u=f, B_{0}(\lambda) u(\cdot, 0)=g$ and $F=(f, g) \in H_{m}^{s}\left(R_{+}^{n+1}\right) \times H_{l}^{s+1 / 2}\left(R^{n}\right)$. Then $(u, u(\cdot, 0)) \in H_{m}^{s}\left(R_{+}^{n+1}\right) \times H_{m}^{s-1 / 2}\left(R^{n}\right)$ when $\lambda \geqq \lambda_{0}$ and $s \geqq 0$. 
Proof. It is enough to prove the theorem when $s$ is integer. $U$ $=(u, u(\cdot, 0))$ is written as $U=L_{0}(\lambda) F$. We put $\chi=\left\{\chi_{j} ; \chi_{j} \equiv 1\right\} 0 \leqq j \leqq k$ in Theorem 5. We have that $U \in X_{0}^{s}(\lambda, \chi)$ because $F \in X_{1}^{s}(\lambda, \chi)$. So, in order to prove the corollary it is enough to show that $\partial_{y}^{s} u \in H_{m}^{0}\left(R_{+}^{n+1}\right)$. Since $A_{0}(\lambda) u=f, \partial_{y}^{s} u$ is written as

$$
\partial_{y}^{s} u=p\left(x, \partial_{x}\right) u+q\left(x, \partial_{x}, \partial_{y}\right) f
$$

where $p\left(x, \partial_{x}\right)$ is a pseudo-differential operator in $x$ of order $s$ and $q\left(x, \partial_{x}, \partial_{y}\right)$ is a pseudo-differential operator in $(x, y)$ of order $s$ (differential operator in $y)$. Thus, $\partial_{y}^{s} u \in H_{m}^{0}\left(R_{+}^{n+1}\right)$.

End of proof.

\section{§5. Existence, Uniqueness and Regularity (Special Case, Continue)}

In this section we shall treat a more general case. It is the case where $M\left(x, \partial_{x}, \lambda\right)$ in the section 4 is $M\left(x, y, \partial_{x}, \lambda\right)$, that is, the pseudodifferential operator in (4.1) may depend on the variable $y$ while the domain is same. The equation is

$$
\left\{\begin{array}{l}
\left\{\frac{\partial}{\partial y}+M\left(x, y, \partial_{x}, \lambda\right)+M_{2}\left(x, y, \partial_{x}, \partial_{y}, \lambda\right)\right\} u=f \\
\left.\left\{B\left(x, \partial_{x}, \lambda\right)+B_{2}\left(x, \partial_{x}, \lambda\right)+B_{3}\left(x, \partial_{x}, \partial_{y}, \lambda\right)\right\} u\right|_{y=0}=g
\end{array}\right.
$$

where $M(x, y, \xi, \lambda)$ and $B(x, \xi, \lambda)$ are homogeneous functions of order 1 and 0 in $(\xi, \lambda)$, respectively, $\{\eta+M(x, 0, \xi, \lambda), B(x, \xi, \lambda)\}$ satisfies <Assumption $(\mathrm{A})>($ Section 2$)$, and $B_{2}\left(x, \partial_{x}, \lambda\right), B_{3}\left(x, \partial_{x}, \partial_{y}, \lambda\right)$ and $M_{2}(x, y$, $\left.\partial_{x}, \partial_{y}, \lambda\right)$ are compositions of pseudo-differential operators in $x$ or $(x, y)$ such that $C_{3}(\lambda) \equiv\left(\begin{array}{l}M_{2}\left(x, y, \partial_{x}, \partial_{y}, \lambda\right), \quad 0 \\ B_{3}\left(x, \partial_{x}, \partial_{y}, \lambda\right), B_{2}\left(x, \partial_{x}, \lambda\right)\end{array}\right)$ is a bounded operator from $Y_{0}^{0}(\lambda)$ to $Y_{1}^{0}(\lambda)$ and satisfies for any $\chi$ and $s$ the estimates that, for $U \in X_{0}^{s}(\lambda, \chi)$ and $j=0, \ldots, k$,

$$
p_{1 j}\left(C_{3}(\lambda) U\right) \leqq c_{0} p_{0 j}(U)+c(s, \chi) \lambda^{-1 / 2} q_{0 j-1}(U),
$$

where $c_{0}$ does not depend on $\lambda, \chi, s, j$ and $k$. (Refer Definition 4.10 for notations.) Moreover we assume that no pseudo-differential operator in the equation $(5.1)$ depends on $(x, y)$ or $x$ outside a ball in $\overline{R_{+}^{n+1}}$ or 
$R^{n}$. We shall show that we can regard (5.1) as a small perturbation of the type dealt with in the section 4 and that we can obtain the same results as ones in the section 4.

\section{Definition 5.1.}

$$
\begin{aligned}
& A_{0}(\lambda) \equiv \frac{\partial}{\partial y}+M\left(x, 0, \partial_{x}, \lambda\right), \\
& A_{1}(\lambda) \equiv \frac{\partial}{\partial y}+M\left(x, y, \partial_{x}, \lambda\right)+M_{2}\left(x, y, \partial_{x}, \partial_{y}, \lambda\right), \\
& A_{2}(\lambda) \equiv \frac{\partial}{\partial y}+M\left(x, y, \partial_{x}, \lambda\right), \\
& B_{0}(\lambda) \equiv B\left(x, \partial_{x}, \lambda\right), \quad B_{1}(\lambda) \equiv B\left(x, \partial_{x}, \lambda\right)+B_{2}\left(x, \partial_{x}, \lambda\right), \\
& B_{2}(\lambda) \equiv B_{2}\left(x, \partial_{x}, \lambda\right), \quad B_{3}(\lambda) \equiv B_{3}\left(x, \partial_{x}, \partial_{y}, \lambda\right), \\
& M_{1}(\lambda) \equiv M\left(x, y, \partial_{x}, \lambda\right)-M\left(x, 0, \partial_{x}, \lambda\right), \quad M_{2}(\lambda) \equiv M_{2}\left(x, y, \partial_{x}, \partial_{y}, \lambda\right) .
\end{aligned}
$$

Lemma 5.1. Let $\varphi \in C_{0}^{\infty}\left(\overline{R_{+}^{1}}\right)$; a function in $y$ and $\varphi(0)=0$. If $u \in W$ (See Definition 4.1.), then $\varphi u \in H_{m}^{1}\left(R_{+}^{n+1}\right)$ and

$$
\left\|\Lambda^{1}(\lambda) \varphi u\right\|_{+} \leqq c\left\{\left\|A_{0}(\lambda) \varphi u\right\|_{+}+\|\varphi u\|_{+}\right\},
$$

where $c$ does not depend on $\varphi$ and $\lambda \geqq 1$.

Proof. Since $u \in W$, so $\varphi u \in W$, too. By Lemma 4.4

$$
\varphi u+\left[S_{1}(\lambda), M\left(x, 0, \partial_{x}, \lambda\right)\right] \varphi u=S_{1}(\lambda) f ; \quad f=A_{0}(\lambda) \varphi u
$$

We apply Lemma 3.12 to this equality. Then, we obtain the estimate we need.

End of proof.

Definition 5.2. $W(A)$ stands for the space such that $u \in W(A)$ if and only if $u$ and $A u \in H_{m}^{0}\left(R_{+}^{n+1}\right)$, where $A$ is an elliptic first order system.

Lemma 5.2. Let $u \in W\left(A_{1}(\lambda)\right)$. Then $u(y) \equiv u(\cdot, y)$ is considered as an $H_{m}^{-1 / 2}\left(R^{n}\right)$-valued continuous function in $y$ on $[0, \infty)$.

Proof. We omit the proof because we can show it in the same way as in Lemma 4.1 if we use the fact that $\Lambda^{-1}(\lambda) \cdot M\left(x, y, \partial_{x}, \lambda\right)$ is a uni- 
formly continuous function in $y \in[0, \infty)$ which is valued in the space of bounded operators on $H_{m}^{0}\left(R^{n}\right)$.

End of proof.

Lemma 5.3. $W\left(A_{0}(\lambda)\right) \equiv W=W\left(A_{1}(\lambda)\right)$.

Proof. From Lemma 5.1 it is clear that $W \subset W\left(A_{1}(\lambda)\right)$, because $A_{1}(\lambda) u=A_{0}(\lambda) u+M_{1}(\lambda) u+M_{2}(\lambda) u$ each term of which is in $H_{m}^{0}\left(R_{+}^{n+1}\right)$ if $u \in W$. We assume that $u$ and $A_{1}(\lambda) u \in H_{m}^{0}\left(R_{+}^{n+1}\right)$. It is enough to show that $\varphi u \in H_{m}^{1}\left(R_{+}^{n+1}\right)$ in order to prove that $W \supset W\left(A_{1}(\lambda)\right)$, where $\varphi \in C_{0}^{\infty}\left(\overline{R_{+}^{1}}\right)$ (a function in $y$ ) and $\varphi(0)=0$, because $A_{0}(\lambda) u=A_{1}(\lambda) u-$ $M_{1}(\lambda) u-M_{2}(\lambda) u$ where $A_{1}(\lambda) u$ and $M_{2}(\lambda) u$ are already in $H_{m}^{0}\left(R_{+}^{n+1}\right)$ and $M_{1}(\lambda) u$ becomes an element in $H_{m}^{0}\left(R_{+}^{n+1}\right)$ if it is shown. It is done by the fact that $A_{1}(\lambda) \varphi u=\left(\frac{\partial}{\partial y} \varphi\right) u+\varphi\left(A_{1}(\lambda)-M_{2}(\lambda)\right) u+M_{2}(\lambda) \varphi u$ $\in H_{m}^{0}\left(R^{n+1}\right)$ on the whole space $R^{n+1}$, where we properly extend $A_{1}(\lambda)$ on $R^{n+1}$ as an elliptic system and $\varphi=u=0$ in $y<0$. And this is verified by using the result of Lemma 5.2 .

End of proof.

Definition 5.3. Let $C_{1}(\lambda)$ stand for the closed operator, from $H_{m}^{0}\left(R_{+}^{n+1}\right) \times H_{m}^{-1 / 2}\left(R^{n}\right)$ to $H_{m}^{0}\left(R_{+}^{n+1}\right) \times H_{l}^{1 / 2}\left(R^{n}\right),\left(\begin{array}{cc}A_{1}(\lambda), & 0 \\ B_{3}(\lambda), & B_{1}(\lambda)\end{array}\right)$ whose definition domain is $W_{b}$.

Remark. It is well-defined because of Lemma 5.3 .

\section{$\S \S 5.1$. Existence of Solutions}

Definition 5.4. Let $R_{0}(\lambda)$ stand for the right inverse $R_{0}(\lambda)$ of $C_{0}(\lambda)$ in Theorem 2 and $P_{1}\left(P_{2}\right)$ stand for the canonical projection from $H_{m}^{0}\left(R_{+}^{n+1}\right) \times H_{m}^{-1 / 2}\left(R^{n}\right)$ to $H_{m}^{0}\left(R_{+}^{n+1}\right)\left(H_{m}^{-1 / 2}\left(R^{n}\right)\right)$ such that $U=\left(u_{1}, u_{2}\right) \rightarrow$ $P_{i} U=u_{i}$. Let us put

$$
R_{0 i}(\lambda) \equiv P_{i} \cdot R_{0}(\lambda)
$$

Lemma 5.4. Let $F=\left(f_{1}, f_{2}\right) \in H_{m}^{0}\left(R_{+}^{n+1}\right) \times H_{l}^{1 / 2}\left(R^{n}\right)$ and $\varphi \in C_{0}^{\infty}\left(\overline{R_{+}^{1}}\right)$; a function in $y$. Then,

$$
\left\|M_{1}(\lambda) \varphi R_{01}(\lambda) F\right\|_{+} \leqq c\left\{\left\|\left(\left|\frac{\partial}{\partial y}(y \varphi)\right|+|y \varphi|\right) R_{01}(\lambda) F\right\|_{+}+\left\|y \varphi f_{1}\right\|_{+}\right\},
$$


where $c$ does not depend on $\varphi$ and $\lambda \geqq \lambda_{0}$.

Proof. We first note that $M_{1}(\lambda)$ is written as $M_{1}(\lambda)=y \tilde{M}(\lambda)$ where $\tilde{M}(\lambda) \cdot \Lambda^{-1}(\lambda)$ is a zero order (bounded) pseudo-differential operator in $x$ with $C_{0}^{\infty}\left(\overline{R_{+}^{n+1}}\right)$-coefficients. We put $u=R_{01}(\lambda) F$. Then, $u \in W$. Using Lemm 5.1, we obtain that

$$
\begin{aligned}
& \left\|M_{1}(\lambda) \varphi R_{01}(\lambda) F\right\|_{+} \\
& \quad \leqq c_{1}\left\|\Lambda^{1}(\lambda) y \varphi R_{01}(\lambda) F\right\|_{+} \\
& \quad \leqq c_{2}\left\{\left\|A_{0}(\lambda) y \varphi R_{01}(\lambda) F\right\|_{+}+\left\|y \varphi R_{01}(\lambda) F\right\|_{+}\right\} \\
& \quad=c_{2}\left\{\left\|\left(\frac{\partial}{\partial y} y \varphi\right) R_{01}(\lambda) F+y \varphi f_{1}\right\|_{+}+\left\|y \varphi R_{01}(\lambda) F\right\|_{+}\right\} \\
& \quad \leqq c_{3}\left\{\left\|\left(\left|\frac{\partial}{\partial y} y \varphi\right|+|y \varphi|\right) R_{01}(\lambda) F\right\|_{+}+\left\|y \varphi f_{1}\right\|_{+}\right\} .
\end{aligned}
$$

End of proof.

Definition 5.5. We may assume that $A_{2}(\lambda)$ is defined as a first order elliptic pseudo-differential system in the whole space $R^{n+1}$. We denote the inverse operator of $A_{2}(\lambda)$ on $H_{m}^{0}\left(R^{n+1}\right)$, whose definition domain is $H_{m}^{1}\left(R^{n+1}\right)$, by $R_{2}(\lambda)$.

Lemma 5.5. There exist some constants $c>0$ and $\lambda_{0}$ such that for any $f \in H_{m}^{0}\left(R^{n+1}\right)$ and $\lambda \geqq \lambda_{0}$ we have that

$$
\left\|\Lambda^{1}(\lambda) \cdot R_{2}(\lambda) f\right\|^{2}+\left\|\frac{\partial}{\partial y} R_{2}(\lambda) f\right\|^{2} \leqq c\|f\|^{2} .
$$

(We omit the proof.)

Let $\psi$ be a function of $C_{0}^{\infty}\left(\overline{R_{+}^{1}}\right)$ in $y$ such that $0 \leqq \psi(y) \leqq 1$ and $\psi(y)=1$ at the neighborhood of $y=0$. Let $u_{1} \equiv \psi R_{01}(\lambda) F, u_{2} \equiv(1-$ $\psi) R_{2}(\lambda) f_{1}$ and $u \equiv u_{1}+u_{2}$, where $F=\left(f_{1}, f_{2}\right) \in H_{m}^{0}\left(R_{+}^{n+1}\right) \times H_{l}^{1 / 2}\left(R^{n}\right)$. We operate $A_{1}(\lambda), B_{3}(\lambda)$ to $u$ and $B_{1}(\lambda)$ to $u(\cdot, 0)$.

$$
\begin{aligned}
& A_{1}(\lambda) u \\
& \quad=A_{1}(\lambda) u_{1}+A_{1}(\lambda) u_{2}
\end{aligned}
$$




$$
\begin{aligned}
= & \left(A_{0}(\lambda)+M_{1}(\lambda)+M_{2}(\lambda)\right) u_{1}+\left(A_{2}(\lambda)+M_{2}(\lambda)\right) u_{2} \\
= & \left(A_{0}(\lambda) \psi\right) R_{01}(\lambda) F+\psi f_{1}+M_{1}(\lambda)\left(\psi R_{01}(\lambda) F\right)+M_{2}(\lambda)\left(\psi R_{01}(\lambda) F\right) \\
& +(1-\psi) A_{2}(\lambda) \cdot R_{2}(\lambda) f_{1}-\left(\frac{\partial}{\partial y} \psi\right) \cdot R_{2}(\lambda) f_{1}+M_{2}(\lambda)\left((1-\psi) R_{2}(\lambda) f_{1}\right) \\
= & f_{1}+\left(\frac{\partial}{\partial y} \psi\right) R_{01}(\lambda) F+M_{1}(\lambda)\left(\psi R_{01}(\lambda) F\right)+M_{2}(\lambda)\left\{\psi R_{01}(\lambda) F\right. \\
& \left.+(1-\psi) R_{2}(\lambda) f_{1}\right\}-\left(\frac{\partial}{\partial y} \psi\right) R_{2}(\lambda) f_{1} \cdot \\
B_{1}(\lambda) u(\cdot, 0) & \quad \text { because } u_{2}(\cdot, 0)=0 \\
= & B_{1}(\lambda) u_{1}(\cdot, 0), \\
= & B_{0}(\lambda) u_{1}(\cdot, 0)+B_{2}(\lambda) u_{1}(\cdot, 0) \\
= & f_{2}+B_{2}(\lambda) \cdot R_{02}(\lambda) F . \\
B_{3}(\lambda) u & \\
= & B_{3}(\lambda) \psi R_{01} F+B_{3}(\lambda)(1-\psi) R_{2}(\lambda) f_{1} .
\end{aligned}
$$

Thus, we obtain the equation (5.2) that $F$ should satisfy in order that $u$ is a solution of (5.1).

Definition 5.6. Let us put

$$
\begin{gathered}
C_{2}(\lambda) \equiv\left(\begin{array}{cc}
M_{1}(\lambda)+M_{2}(\lambda), & 0 \\
B_{3}(\lambda) & , B_{2}(\lambda)
\end{array}\right), \quad C_{3}(\lambda) \equiv\left(\begin{array}{cc}
M_{2}(\lambda), & 0 \\
B_{3}(\lambda), & B_{2}(\lambda)
\end{array}\right) \\
R_{2}(\lambda) \equiv\left(\begin{array}{r}
R_{2}(\lambda), 0 \\
0,0
\end{array}\right), \quad \Psi \equiv\left(\begin{array}{c}
\psi, 0 \\
0, I
\end{array}\right) \text { and } \Psi_{y} \equiv\left(\begin{array}{cc}
\frac{\partial}{\partial y} \psi, & 0 \\
0 & , 0
\end{array}\right) . \\
F+T_{1}(\lambda) F=G,
\end{gathered}
$$

where $F=\left(f_{1}, f_{2}\right), G=(f, g)$, which is one given in (5.1), and (5.3) $\quad T_{1}(\lambda) \equiv C_{2}(\lambda) \cdot \Psi \cdot R_{0}(\lambda)+\Psi_{y} \cdot R_{0}(\lambda)+C_{3}(\lambda) \cdot(I-\Psi) \cdot R_{2}(\lambda)-\Psi_{y}^{\prime} \cdot R_{2}(\lambda)$. 
Lemma 5.6. Let $F \in H_{m}^{0}\left(R_{+}^{n+1}\right) \times H_{l}^{1 / 2}\left(R^{n}\right)=Y_{1}^{0}(\lambda)$. For any $a(0<a<1)$ there exist $\psi$ and $\lambda_{0}$ such that, for $\lambda \geqq \lambda_{0}$,

$$
\left\|T_{1}(\lambda) F\right\| \leqq a\|F\|
$$

where $\|F\| \equiv\|F\|_{Y_{1}^{0}(\lambda)}=\left(\left\|f_{1}\right\|_{+}^{2}+\left\|\Lambda^{1 / 2}(\lambda) f_{2}\right\|^{2}\right)^{1 / 2} ; F=\left(f_{1}, f_{2}\right)$.

Proof. Let $|\varphi|_{+} \equiv \max _{y \in[0, \infty)}|\varphi(y)|$ for $\varphi \in C_{0}^{\infty}\left(\overline{R_{+}^{1}}\right)$. Then,

$$
\left\|M_{1}(\lambda) \cdot \psi \cdot R_{01}(\lambda) F\right\|_{+} \leqq \alpha_{1}\left\{\left(\left|\frac{\partial}{\partial y} y \psi\right|_{+}+|y \psi|_{+}\right) \lambda^{-1}+|y \psi|_{+}\right\}\|F\|
$$

because of Lemma 5.4 and Theorem 2 .

$$
\begin{aligned}
& \left\|C_{3}(\lambda) \cdot\left(\Psi \cdot R_{0}(\lambda)+(I-\Psi) \cdot R_{2}(\lambda)\right) F\right\| \\
& \quad \leqq \alpha_{2}^{\prime}\left\|\left(\Psi \cdot R_{0}(\lambda)+(I-\Psi) R_{2}(\lambda)\right) F\right\| \leqq \alpha_{2} \lambda^{-1}\|F\|
\end{aligned}
$$

because of the definition of $C_{3}(\lambda)$, Theorem 2 and Lemma 5.5 .

$$
\begin{aligned}
\left\|\Psi_{y} \cdot\left(R_{0}(\lambda)-R_{2}(\lambda)\right) F\right\| \\
\quad=\left\|\left(\frac{\partial}{\partial y} \psi\right)\left(R_{01}(\lambda)-R_{2}(\lambda)\right) F\right\|_{+} \leqq \alpha_{3} \lambda^{-1}\left|\frac{\partial}{\partial y} \psi\right|_{+}\|F\|
\end{aligned}
$$

because of Theorem 2 and Lemma 5.5.

Thus, there exist $\psi$ and $\lambda_{0}$ such that for $\lambda \geqq \lambda_{0}\left\|T_{1}(\lambda)\right\| \leqq a<1$, that is, $\alpha_{1}|y \psi|_{+}+\left\{\alpha_{1}\left(\left|\frac{\partial}{\partial y} y \psi\right|_{+}+|y \psi|_{+}\right)+\alpha_{2}+\alpha_{3}\left|\frac{\partial}{\partial y} \psi\right|_{+}\right\} \lambda^{-1} \leqq a<1$. In fact there exists $\psi$ such that $\alpha_{1}|y \psi|_{+}<a / 2$ because $|y \psi|_{+}<\varepsilon$ when the support of $\psi$ is contained in $[0, \varepsilon]$, and we put for such a $\psi$ fixed $\lambda_{0} \equiv 2\left\{\alpha_{1}\left(\left|\frac{\partial}{\partial y} y \psi\right|_{+}\right.\right.$ $\left.\left.+|y \psi|_{+}\right)+\alpha_{2}+\alpha_{3}\left|\frac{\partial}{\partial y} \psi\right|_{+}\right\} a^{-1}$.

End of proof.

Theorem 6. There exists a bounded operator $R_{1}(\lambda)$ from $H_{m}^{0}\left(R_{+}^{n+1}\right)$ $\times H_{l}^{1 / 2}\left(R^{n}\right)$ to $H_{m}^{0}\left(R_{+}^{n+1}\right) \times H_{m}^{-1 / 2}\left(R^{n}\right)$ for $\lambda \geqq \lambda_{0}$ such that the range of $R_{1}(\lambda)$ is contained in $W_{b},\left\|R_{1}(\lambda)\right\| \leqq c \lambda^{-1}$ and $C_{1}(\lambda) \cdot R_{1}(\lambda)=I$ on $H_{m}^{0}\left(R_{+}^{n+1}\right)$ $\times H_{l}^{1 / 2}\left(R^{n}\right)$.

Proof. There exists the inverse operator of $\left(I+T_{1}(\lambda)\right)$ for $\lambda \geqq \lambda_{0}$ because of Lemma 5.6. We now put 


$$
R_{1}(\lambda) \equiv\left\{\Psi \cdot R_{0}(\lambda)+(I-\Psi) \cdot R_{2}(\lambda)\right\} \cdot\left(I+T_{1}(\lambda)\right)^{-1}
$$

The previous discussion shows that this $R_{1}(\lambda)$ is what we need. The norm of $R_{1}(\lambda)$ is obtained by combining Theorem 2, Lemma 5.5 and Lemma 5.6.

End of proof.

\section{$\S \S 5.2$. Uniqueness of Solution}

Let $U \in W_{b}$ (See Definition 4.2.) $\subset H_{m}^{0}\left(R_{+}^{n+1}\right) \times H_{m}^{-1 / 2}\left(R^{n}\right)$ and satisfy (5.1). We operate $L_{0}(\lambda)$, which is a left inverse of $C_{0}(\lambda)$, that is, $\left(\begin{array}{c}A_{0}(\lambda) \text {, } \\ 0\end{array}\right.$, $\left.\begin{array}{c}0 \\ B_{0}(\lambda)\end{array}\right)$ whose definition domain is $W_{b}$ (See Theorem 3.), to (5.1), that is, $C_{1}(\lambda) U=F$.

$$
\begin{aligned}
L_{0}(\lambda) F & =L_{0}(\lambda) \cdot C_{1}(\lambda) U \\
& =L_{0}(\lambda) \cdot C_{0}(\lambda) U+L_{0}(\lambda) \cdot C_{2}(\lambda) U \\
& =U+L_{0}(\lambda) \cdot C_{2}(\lambda) U
\end{aligned}
$$

Let $\left.\varphi=C^{\infty} \overline{\left(R_{+}^{1}\right.}\right), 0<\varphi(y) \leqq 1(y>0), \varphi(y)=y(0 \leqq y \leqq 1 / 2$.$) and \varphi(y)=1(y$ $>1)$. Then, $M_{1}(\lambda)=\tilde{M}(\lambda) \varphi ; \tilde{M}(\lambda)$ is a first order pseudo-differential system with $C_{0}^{\infty}\left(\overline{R_{+}^{n+1}}\right)$ coefficients. Since $A_{2}(\lambda) \varphi u=\left(\frac{\partial}{\partial y} \varphi\right) u-\varphi M_{2}(\lambda) u+\varphi f$ on $R^{n+1}$, where $U=\left(\begin{array}{l}u \\ \left.u\right|_{y=0}\end{array}\right)$ and $\varphi=u=f=0$ in $y<0$, we have that $\varphi u=R_{2}(\lambda)\left(\frac{\partial}{\partial y} \varphi\right) u-R_{2}(\lambda) \cdot \varphi M_{2}(\lambda) u+R_{2}(\lambda) \varphi f . \quad$ (See Definition 5.4.) Thus, we put it in (5.7).

$$
\begin{aligned}
U & +L_{0}(\lambda) \cdot \tilde{M}(\lambda) \cdot R_{2}(\lambda) \cdot\left\{\Phi_{y}-\Phi \cdot C_{3}(\lambda)\right\} U+L_{0}(\lambda) \cdot C_{3}(\lambda) U \\
& =L_{0}(\lambda) F-L_{0}(\lambda) \cdot \tilde{M}(\lambda) \cdot R_{2}(\lambda) \cdot \Phi F
\end{aligned}
$$

where $\Phi \equiv\left(\begin{array}{c}\varphi, 0 \\ 0,0\end{array}\right), \Phi_{y} \equiv\left(\begin{array}{c}\frac{\partial}{\partial y} \varphi, 0 \\ 0,0\end{array}\right)$ and we identify $\tilde{M}(\lambda) \cdot R_{2}(\lambda)$ to $\left(\begin{array}{cr}\tilde{M}(\lambda) \cdot R_{2}(\lambda), & 0 \\ 0 & , 0\end{array}\right) . \quad$ Let us put

$$
T_{2}(\lambda) \equiv L_{0}(\lambda) \cdot \tilde{M}(\lambda) \cdot R_{2}(\lambda) \cdot\left\{\Phi_{y}-\Phi \cdot C_{3}(\lambda)\right\}+L_{0}(\lambda) \cdot C_{3}(\lambda)
$$


Lemma 5.7. $\left\|T_{2}(\lambda)\right\| \leqq c \lambda^{-1} \quad$ on $\quad H_{m}^{0}\left(R_{+}^{n+1}\right) \times H_{m}^{-1 / 2}\left(R^{n}\right)=Y_{0}^{0}(\lambda)$ for $\lambda \geqq \lambda_{0}$.

Proof. $\left\|C_{3}(\lambda) U\right\|_{Y_{1}^{0}(\lambda) \leqq c\|U\|_{Y_{0}^{0}(\lambda)}}$ by the assumption. $\| \tilde{M}(\lambda) \cdot R_{2}(\lambda) \cdot$ $\left\{\Phi_{y}-\Phi \cdot C_{3}(\lambda)\right\} U\left\|_{Y_{1}^{0}(\lambda)}=\right\| \tilde{M}(\lambda) \cdot R_{2}(\lambda)\left\{\frac{\partial}{\partial y} \varphi-\varphi \cdot M_{2}(\lambda)\right\} u\left\|_{+} \leqq c\right\| u \|_{+} \leqq$ $c\|U\|_{Y_{0}^{0}(\lambda)}$ by Lemma 5.5, and $\left\|L_{0}(\lambda) V\right\|_{Y_{0}^{0}(\lambda)} \leqq c \lambda^{-1}\|V\|_{Y_{1}^{0}(\lambda)}$ by Theorem 3, where $\|\cdot\|_{Y_{i}^{0}(\lambda)}$ stands for the norm with the parameter $\lambda$ of $H_{m}^{0}\left(R_{+}^{n+1}\right)$ $\times H_{\alpha_{i}}^{-1 / 2+i}\left(R^{n}\right), \alpha_{0}=m, \alpha_{1}=l$. (See Definition 4.10.) Combining these three, we obtain the estimate of this lemma.

End of proof.

Theorem 7. For any $\lambda \geqq \lambda_{0}$ ( $\lambda_{0}$ is a fixed constant) there exists a bounded operator $L_{1}(\lambda)$ from $H_{m}^{0}\left(R_{+}^{n+1}\right) \times H_{l}^{1 / 2}\left(R^{n}\right)$ to $H_{m}^{0}\left(R_{+}^{n+1}\right) \times$

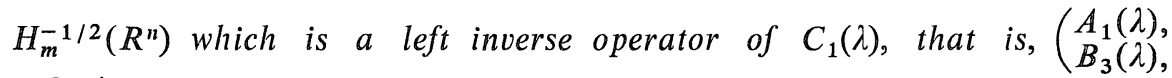
$\left.\begin{array}{c}0 \\ B_{1}(\lambda)\end{array}\right)$ whose definition domains is $W_{b} \subset H_{m}^{0}\left(R_{+}^{n+1}\right) \times H_{m}^{-1 / 2}\left(R^{n}\right)$ and satisfies the estimate $\left\|L_{1}(\lambda)\right\| \leqq c \lambda^{-1}$.

Proof. By Lemma 5.7 there exists a constant $\lambda_{0}$ such that for $\lambda \geqq \lambda_{0}$ there exists $\left(I+T_{2}(\lambda)\right)^{-1}$ on $H_{m}^{0}\left(R_{+}^{n+1}\right) \times H_{m}^{-1 / 2}\left(R^{n}\right)$. We put

$$
L_{1}(\lambda) \equiv\left(I+T_{2}(\lambda)\right)^{-1} \cdot L_{0}(\lambda) \cdot\left(I-\tilde{M}(\lambda) \cdot R_{2}(\lambda) \cdot \Phi\right) .
$$

This is what we need, that is, $L_{1}(\lambda) \cdot C_{1}(\lambda)=I$ and the estimate is obtained from the estimate of $L_{0}(\lambda)$.

End of proof.

\section{§5.3. Regularity of Solutions}

We can easily show the regularity in $x$ of solutions by the similar way as in the section 4 . We use the equation (5.8).

Lemma 5.8. $R_{2}(\lambda)$ satisfies the estimate for $u$ such that $(u, 0)$ $\in X_{i}^{s}(\lambda, \chi), 0 \leqq s \leqq k / 2$. (See Definition 4.10.)

$$
p_{i j}\left(\Lambda^{1}(\lambda) \cdot R_{2}(\lambda) u, 0\right) \leqq c_{0} p_{i j}(u, 0)+c(s, \chi) \lambda^{-1 / 2} q_{i j-1}(u, 0),
$$

where $c_{0}$ is a constant not depending on $\lambda, s$ and $\chi$.

Proof. We remark that it is enough to obtain the following type 
of estimate (5.11) for $\mathscr{T}=\Lambda^{1}(\lambda) \cdot R_{2}(\lambda)$ and $0 \leqq s \leqq k$.

$$
\begin{aligned}
& \left\|\Lambda^{s}(\Lambda) \chi_{k} \mathscr{T} u\right\|^{2} \\
& \leqq c_{0}\left\|\Lambda^{s}(\lambda) \chi_{k} u\right\|^{2}+c(s, \chi) \lambda^{-1 / 2} \sum_{j=0}^{k-1}\left\|\Lambda^{s-(k-j) / 2}(\lambda) \chi_{j} u\right\|^{2}
\end{aligned}
$$

for $u \in C_{0}^{\infty}\left(R^{n+1}\right)$ and $\lambda \geqq \lambda_{0}$, where $\left\{\chi_{j}\right\}_{j=0, \ldots, k}=\chi, \chi_{j}(y)=\chi_{j}(-y)$ if $y<0$, $\|\cdot\|$ is the norm of $H_{m}^{0}\left(R^{n+1}\right)$, and $\lambda_{0}$ and $c_{0}$ are constants not depending on $s$ and $\chi$. Let $S^{\prime}(\lambda)$ stand for the pseudo-differential operators in $R^{n+1}$ whose symbol is $A_{2}^{-1}(x, y, \xi, \eta, \lambda) \equiv\{i \eta+M(x, y, \breve{\zeta}, \lambda)\}^{-1}$ and let $T^{\prime}(\lambda) \equiv\left[A_{2}(\lambda), S^{\prime}(\lambda)\right]$. Then we can represent $R_{2}(\lambda)$ as

$$
R_{2}(\lambda)=S^{\prime}(\lambda) \cdot U^{\prime}(\lambda), \quad \text { where } \quad\left(I+T^{\prime}(\lambda)\right) U^{\prime}(\lambda)=I
$$

on $H_{m}^{0}\left(R^{n+1}\right)$. From Lemma 3.18, we have the estimate (5.11) for $\mathscr{T}$ $=\Lambda^{1}(\lambda) \cdot S^{\prime}(\lambda)$ or $\lambda T^{\prime}(\lambda)$ and $0 \leqq s \leqq k / 2$. So, we have it for $\mathscr{T}=U^{\prime}(\lambda)$ by Lemma 4.9. Combining these, we obtain the estimate for $R_{2}(\lambda)$.

End of proof.

Lemma 5.9. When $0 \leqq s \leqq k / 2, \lambda \geqq \lambda_{0}$ and $0 \leqq j \leqq k, T_{2}(\lambda)$ (See (5.9.)) satisfies the estimates that, for $U \in X_{0}^{s}(\lambda, \chi)$,

$$
p_{0 j}\left(T_{2}(\lambda) U\right) \leqq c_{0} \lambda^{-1} p_{0 j}(U)+c(s, \chi) \lambda^{-3 / 2} q_{0 j-1}(U),
$$

where $c_{0}$ and $\lambda_{0}$ are constants not depending on $s, \lambda$ and $\chi$.

Proof. It is the combination of Theorem 5, Lemma 5.8 and the assumption for $C_{3}(\lambda)$.

End of proof.

Theorem 8. There exists a constant $\lambda_{0}$, which does not depend on $\lambda, s$ and $\chi$, such that the inverse operator $L_{1}(\lambda)$ in Theorem 7 is a bounded operator from $X_{1}^{s}(\lambda, \chi)$ to $X_{0}^{s}(\lambda, \chi)$ when $\lambda \geqq \lambda_{0}$ and $0 \leqq s \leqq k / 2$. $L_{1}(\lambda)$ satisfies the estimate for $F \in X_{1}^{s}(\lambda, \chi)$ and $0 \leqq j \leqq k$ that

$$
p_{0 j}\left(L_{1}(\lambda) F\right) \leqq c_{0} \lambda^{-1} p_{1 j}(F)+c(s, \chi) \lambda^{-3 / 2} q_{1 j-1}(F)
$$

where $c_{0}$ is a constant not depending on $s \geqq 0, \lambda \geqq \lambda_{0}$ and $\chi$.

Proof. We apply $T_{2}(\lambda)$ to Lemma 4.9 because of Lemma 5.9. We obtain that $\left(I+T_{2}(\lambda)\right)^{-1}$ is a bounded operator on $X_{0}^{s}(\lambda, \chi)$ when we 
put $\lambda_{0}>1 / c_{0}$. We combine it with Lemma 5.8. Then, we obtain the results of Theorem 8 from the definition of $L_{1}(\lambda)$. (See (5.10).)

End of proof.

Let us put $M^{(0)}(\lambda) \equiv M(\lambda)+M_{2}(\lambda)$

$$
\begin{aligned}
M^{(i)}(\lambda) & \equiv\left[\frac{\partial}{\partial y}, M^{(i-1)}(\lambda)\right] \\
& =\frac{\partial}{\partial y} \cdot M^{(i-1)}(\lambda)-M^{(i-1)}(\lambda) \cdot \frac{\partial}{\partial y} \\
& =\left(\left(\frac{\partial}{\partial y}\right)^{i} M^{(0)}\right)\left(x, y, \partial_{x}, \partial_{y}, \lambda\right), \quad i=1,2, \ldots
\end{aligned}
$$

We furthermore assume for $M^{(i)}(\lambda)$ to satisfy the additional conditions (5.12). Then, we have the following theorem for regularity of solutions, which will be used for proof of theorem in general cases.

$$
\begin{aligned}
& \text { For } i=0,1,2, \ldots, \quad s, t \in R^{1} \quad \text { and } \quad s \geqq 0, \\
& p_{1 j}\left(\Lambda^{t-1-i}(\lambda) v, 0\right) \\
& \leqq c_{j} p_{0 j}\left(\Lambda^{t}(\lambda) u, 0\right)+c(s, \chi, t, i) \lambda^{-1 / 2} q_{0 j}\left(\Lambda^{t}(\lambda) u, 0\right),
\end{aligned}
$$

if $v=M^{(i)}(\lambda) u, \Lambda^{t}(\lambda) u \in X_{0}^{s}(\lambda, \chi), j=0, \ldots, k$ and $s \leqq k / 2$.

Remark. It is clear that $M(\lambda)$ satisfies a stronger form of inequality (5.12), and $M_{2}(\lambda)$ the lower term which we shall deal with in the next section. In fact, we set $M_{2}^{(0)}(\lambda)=M_{2}(\lambda)$ and $M_{2}^{(i)}(\lambda) \equiv\left[\frac{\partial}{\partial y}, M_{2}^{(i-1)}(\lambda)\right]=$ $=\left(\frac{\partial}{\partial y}\right)^{i} M_{2}(\lambda)$. Then, $C_{3}(\lambda)$ and $M_{2}^{(i)}(\lambda)$ satisfy (5.2) and (5.12) if they satisfy (5.13), (5.14) and (5.15). For any real number $s \geqq 0$ and $\chi_{i}$ $(i=1,2)$ in Lemma 3.14 ,

$$
\begin{aligned}
& \left\|\Lambda^{s}(\lambda) v_{1}\right\|_{+}^{2}+\left\|\Lambda^{s+1 / 2}(\lambda) v_{2}\right\|^{2} \\
& \leqq c_{s}\left(\left\|\Lambda^{s}(\lambda) u_{1}\right\|_{+}^{2}+\left\|\Lambda^{s-1 / 2}(\lambda) u_{2}\right\|^{2}\right) \\
& \left\|\Lambda^{s}(\lambda) \chi_{2} v_{1}\right\|_{+}^{2}+\left\|\Lambda^{s+1 / 2}(\lambda) \chi_{2} v_{2}\right\|^{2} \\
& \leqq c_{0}\left(\left\|\Lambda^{s}(\lambda) \chi_{2} u_{1}\right\|_{+}^{2}+\left\|\Lambda^{s-1 / 2}(\lambda) \chi_{2} u_{2}\right\|^{2}\right)
\end{aligned}
$$




$$
\begin{aligned}
+c\left(\left\|\Lambda^{s-1}(\lambda) \chi_{1} u_{1}\right\|_{+}^{2}\right. & +\left\|\Lambda^{s-3 / 2}(\lambda) \chi_{1} u_{2}\right\| \\
& \left.+\left\|u_{1}\right\|_{+}^{2}+\left\|\Lambda^{-1 / 2}(\lambda) u_{2}\right\|^{2}\right)
\end{aligned}
$$

$$
\begin{aligned}
& \left\|\Lambda^{s}(\lambda) M_{2}^{(i)}(\lambda) u_{1}\right\|_{+} \leqq c_{s i}\left\|\Lambda^{s}(\lambda) u_{1}\right\|_{+} \\
& \left\|\Lambda^{s}(\lambda) \chi_{2} M_{2}^{(i)}(\lambda) u_{1}\right\|_{+} \leqq \\
& \quad c_{s i}\left\|\Lambda^{s}(\lambda) \chi_{2} u_{1}\right\|_{+} \\
& \quad+c\left(\left\|\Lambda^{s-1}(\lambda) \chi_{1} u_{1}\right\|_{+}+\left\|u_{1}\right\|_{+}\right),
\end{aligned}
$$

where we set $V=\left(\begin{array}{l}v_{1} \\ v_{2}\end{array}\right)=C_{3}(\lambda) U$ and $U=\left(\begin{array}{l}u_{1} \\ u_{2}\end{array}\right)$

Theorem 9. We assume the additional condition (5.12) in Theorem 8. Let $F=(f, g)$ and $\left(\Lambda^{-v}(\lambda) \partial_{y}^{v} f, 0\right) \in X_{1}^{s}(\lambda, \chi)(0 \leqq v \leqq s+1)$. Then $\left(\Lambda^{-v}(\lambda) \partial_{y}^{v} u, 0\right) \in X_{0}^{s}(\lambda, \chi)$ for $0 \leqq v \leqq s+1$ and

$$
\begin{aligned}
& p_{0 j}\left(\Lambda^{-v}(\lambda) \partial_{y}^{v} u, 0\right) \\
& \leqq \\
& c_{v} \lambda^{-1}\left\{\sum_{i=0}^{v-1} p_{1 j}\left(\Lambda^{-i}(\lambda) \partial_{y}^{i} f, 0\right)+p_{1 j}(0, g)\right\} \\
& \quad+c(v, s, \chi) \lambda^{-3 / 2}\left\{\sum_{i=0}^{v-1} q_{1 j-1}\left(\Lambda^{-i}(\lambda) \partial_{y}^{i} f, 0\right)+q_{1 j-1}(0, g)\right\}
\end{aligned}
$$

Proof. This is a corollary of Theorem 8. We use the induction in $0 \leqq v \leqq s+1$. Since $\left\{\frac{\partial}{\partial y}+M^{(0)}(\lambda)\right\} u=f$, we have that

$$
\left(\frac{\partial}{\partial y}\right)^{v+1} u=-\sum_{i=0}^{v} c_{v i} M^{(i)}(\lambda)\left(\frac{\partial}{\partial y}\right)^{v-i} u+\left(\frac{\partial}{\partial y}\right)^{v} f
$$

$\left(\Lambda^{-v}(\lambda)\left(\frac{\partial}{\partial y}\right)^{v} f, 0\right)$ and $\left(\Lambda^{i-v}(\lambda)\left(\frac{\partial}{\partial y}\right)^{v-i} u, 0\right) \in X_{0}^{s}(\lambda, \chi)$ by assumption. $\left(\Lambda^{-(v+1)}(\lambda) M^{(i)}(\lambda)\left(\frac{\partial}{\partial y}\right)^{v-i} u, 0\right) \in X_{0}^{s}(\lambda, \chi)$ and are bounded by the right hand side of (5.16) because of (5.12). Thus, $\left(\Lambda^{-(v+1)}(\lambda)\left(\frac{\partial}{\partial y}\right)^{v+1} u, 0\right)$ $\in X_{0}^{s}(\lambda, \chi)$ and satisfies (5.16).

End of proof.

Corollary 1 of Theorem 9. Let $(f, g) \in H_{m}^{s}\left(R_{+}^{n+1}\right) \times H_{l}^{s+1 / 2}\left(R^{n}\right)$ and $\left(\chi f, \chi^{0} g\right) \in H_{m}^{s+1 / 2}\left(R_{+}^{n+1}\right) \times H_{l}^{s+1}\left(R^{n}\right)$. Then, $(u, u(0)) \in H_{m}^{s}\left(R_{+}^{n+1}\right) \times H_{m}^{s-1 / 2}$ $\left(R^{n}\right)$ and $\left(\chi u, \chi^{0} u(0)\right) \in H_{m}^{s+1 / 2}\left(R_{+}^{n+1}\right) \times H_{m}^{s}\left(R^{n}\right)$ if $s \geqq 0, \chi \in C_{0}^{\infty}\left(\overline{R_{+}^{n+1}}\right)$ and 
$\chi^{0}=\left.\chi\right|_{y=0} \in C_{0}^{\infty}\left(R^{n}\right)$

Corollary 2 of Theorem $9 . R_{1}(\lambda)\left(=L_{1}(\lambda)\right)$ satisfies the estimate (5.18) for $\lambda \geqq \lambda_{0}$ and $s \geqq 0$ as operator from $H_{m}^{s}\left(R_{+}^{n+1}\right) \times H_{l}^{s+1 / 2}\left(R^{n}\right)$ to $H_{m}^{s}\left(R_{+}^{n+1}\right) \times H_{m}^{s-1 / 2}\left(R^{n}\right)$.

$$
\left\|R_{1}(\lambda)\right\| \leqq c_{s} \lambda^{-1}
$$

where we use the norm $\left\|\left(\Lambda^{1}(\lambda)-i \partial_{y}\right)^{s} u\right\|_{+}$for $u \in H^{s}\left(R_{+}^{n+1}\right)$ and $\left\|\Lambda^{s}(\lambda) v\right\|$ for $v \in H^{s}\left(R^{n}\right)$.

\section{§6. Proof of Theorem 1}

We here use the symbols, the notations and the assumptions in the section 1. We assume that $(A, B)$ is already the remodeled system of first order with a weight index $(r, s, t)$. We can construct a local right or left parametrix by Theorems 6,7 or 9 , because there exists a partition of unity of class $C^{\infty}$ which satisfies proper conditions (Lemma 6.1), and we shall show that the error term is a small perturbation and that the regularity of solutions is immediately deduced by Theorem 9 .

Lemma 6.1. There exist a system of finite number non-negative real valued functions $\left\{\alpha_{i}, \beta_{i}\right\}$ and a system of finite number local coordinates $\left\{\left(\Omega^{v}, x^{v}\right)\right\}$ of the compact domain $\bar{\Omega}$ with $C^{\infty}$ boundary such that 1) $\left.\sum \alpha_{v}^{2}=1, \alpha_{v} \beta_{v}=\alpha_{v}, \operatorname{supp} \beta_{v} \subset \Omega^{v}, 2\right) x^{v}\left(\Omega^{v}\right)=V^{n+1}$ if $\Omega^{v} \cap \partial \Omega=\phi$, and $x^{v}\left(\Omega^{v}\right)=V_{+}^{n+1}$ if $\Omega^{v} \cap \partial \Omega \neq \phi$, where $V^{n+1}$ is the open unit ball in $R^{n+1}$ and $V_{+}^{n+1}=V^{n+1} \cap \overline{R_{+}^{n+1}}$, and 3) the partial differential system $(A, B)$ is an elliptic system properly (strongly) linked by $\lambda$ on $\Omega^{v}$ for $a$ weight index $(r, s, t)$ and a local coordinate function $\left(x^{v}\right)$.

(We omit the proof.)

Lemma 6.2. Let us put $\left(A_{v}, B_{v}\right)=\left.(A, B)\right|_{\operatorname{supp} \beta_{v}}$. We can extend $\left(A_{v}, B_{v}\right)$ in $\overline{R_{+}^{n+1}}$, if $\Omega^{v} \cap \partial \Omega \neq \phi$ (in $R^{n+1}$ if $\left.\Omega^{v} \cap \partial \Omega=\phi\right)$, as the extended system keeps to be an elliptic system properly (strongly) linked by $\lambda$ and does not depend on $x^{v}$ outside a bounded set. We denote it by $\left(\hat{A}_{v}, \hat{B}_{v}\right)$. 
Proof. Let $\varphi=C_{0}^{\infty}\left(\overline{R_{+}^{1}}\right)$ such that $0 \leqq \varphi \leqq 1, \varphi(t)=1$ on $0 \leqq t \leqq$ $\sup \left\{\left|x^{v}\right| ; x^{v} \in \operatorname{supp} \beta_{v}\right\}<1$ and $\varphi(t)=0$ on $t \geqq 1$. If we replace $x^{v}$ with $x^{v} \varphi\left(\left|x^{v}\right|\right)$, we obtain the systems $\hat{A}_{v}$ and $\hat{B}_{v}$ on $\overline{R_{+}^{n+1}}$ (or $R^{n+1}$ ) and $R^{n}$, and we can show that $\left(\hat{A}_{v}, \hat{B}_{v}\right)$ satisfies the above conditions. End of proof.

We use the symbol $(\hat{A}, \widehat{B})$ for $\left(\widehat{A}_{v}, \widehat{B}_{v}\right)$. We omit the index $v$ since we are not confused without it. Let us define $\mathscr{N}_{i}$ as well as (1.2).

$$
\begin{aligned}
& \mathscr{N}_{0} \equiv \mathscr{N}_{0}(\mu, \zeta) \equiv\left((|\zeta|-\mu)^{-r_{J}+r_{0}} \delta_{i j}\right) \\
& \mathscr{N}_{1} \equiv \mathscr{N}_{1}(\mu, \zeta) \equiv\left((|\zeta|-\mu)^{-s_{j}-r_{0}+1} \delta_{i j}\right) \\
& \mathscr{N}_{2} \equiv \mathscr{N}_{2}(\zeta) \equiv\left(|\zeta|^{-t_{J}-r_{0}} \delta_{i j}\right) \\
& \mathscr{N}_{3} \equiv \mathscr{N}_{3}(\zeta) \equiv\left(|\zeta|^{-r_{j}+r_{0}} \delta_{i j}\right)
\end{aligned}
$$

where $\left(\delta_{i j}\right)$ is Kronecker's $\delta$ and $\zeta=(\xi, \lambda)$. (Refer (1.2).) And let us denote the pseudo-differential operators whose symbols are $\mathscr{N}_{i}$ by $N_{i}(i=0,1,2$ and 3), where we consider the Fourier inverse transform in $\xi$ and the Laplace inverse transform in $\mu$ whose integral is taken on the pure imaginary axis.

Lemma 6.3. $\quad N_{i}(i=0,1,2$ or 3$)$ is an isomorphism from $X$ to $Y$, where $X=\prod_{j=1}^{m} H^{\sigma_{i j}+\tau_{j}}\left(R_{+}^{n+1}\right), Y=\prod_{j=1}^{m} H^{\tau_{j}}\left(R_{+}^{n+1}\right), \tau=\left(\tau_{j}\right)$ a system of real numbers, $\sigma_{l_{i}} \sigma_{0 j}=r_{0}-r_{j}$ and $\sigma_{1 j}=1-r_{0}-s_{j}$ if $i=0$ or 1 , or $X=\prod_{j=1}^{l_{i}} H^{\sigma_{i j}+\tau_{j}}\left(R^{n}\right)$ $Y=\prod_{j=1}^{l_{i}} H^{\tau_{J}}\left(R^{n}\right), \sigma_{2 j}=-r_{0}-t_{j}, \sigma_{3 j}=r_{0}-r_{j}, l_{2}=l$ and $l_{3}=m$ if $i=2$ or 3 .

(We omit the proof. Refer Lemma 3.19.)

Definition 6.1. Let us put as $\tilde{A} \equiv N_{1} \hat{A} N_{0}, \widetilde{B}^{\prime} \equiv N_{2} \widehat{B} N_{0} \equiv \sum_{k=0}^{\tilde{r}_{0}}$ $\tilde{B}_{k}^{\prime}\left(\frac{\partial}{\partial y}\right)^{k}, \tilde{A}_{0} \equiv \hat{\mathscr{A}}_{0}^{(0)}$ (See Definition 1.1), $\tilde{M} \equiv \frac{\partial}{\partial y}-\left(\tilde{A}_{0}\right)^{-1} \tilde{A}, \tilde{M}_{0} \equiv I, \tilde{M}_{k}$ $\equiv\left[\frac{\partial}{\partial y}, \tilde{M}_{k-1}\right]+\tilde{M}_{k-1} \tilde{M}, \tilde{M}_{0}^{\prime} \equiv 0, \tilde{M}_{k}^{\prime} \equiv \frac{\partial}{\partial y} \tilde{M}_{k-1}^{\prime}+\tilde{M}_{k-1},(k=1,2, \ldots), \tilde{B} \equiv$ $\sum_{k=0}^{\tilde{r}_{0}} \widetilde{B}_{k}^{\prime} \tilde{M}_{k}$ and $\widetilde{B}^{\prime \prime} \equiv \sum_{k=0}^{\tilde{r}_{0}} \widetilde{B}_{k}^{\prime} \tilde{M}_{k}^{\prime}$, where $\tilde{r}_{0} \equiv \max _{i}\left\{r_{0}-r_{i}\right\}, \widetilde{B}_{k}^{\prime}$ are compositions of pseudo-differential operators in $x$ with parameter $\lambda$ and we define only $\widetilde{A}$ if $\Omega_{v} \cap \partial \Omega=\phi$. Let $\widetilde{A}^{(0)}, \widetilde{B}^{(0)^{\prime}}, \tilde{M}^{(0)}, \widetilde{B}^{(0)}, \tilde{A}^{(1)}$ and $\tilde{M}^{(1)}$ stand for the pseudo-differential operators whose symbols are $\tilde{\mathscr{A}}^{(0)}, \widetilde{\mathscr{B}}^{(0)^{\prime}}, \tilde{\mathscr{M}}^{(0)}, \widetilde{\mathscr{B}}^{(0)}$, $\widetilde{\mathscr{A}}^{(1)}$ and $\tilde{\mathscr{M}}^{(1)}$, respectively', where $\widetilde{\mathscr{A}}^{(0)} \equiv \mathscr{N}_{1} \hat{\mathscr{A}}^{(0)} \mathscr{N}_{0}, \widetilde{\mathscr{B}}^{(0)^{\prime}} \equiv \mathscr{N}_{2} \hat{\mathscr{B}}^{(0)} \mathscr{N}_{0}$ 
$=\sum_{k=0}^{\tilde{r}_{0}} \widetilde{\mathscr{B}}_{k}^{(0) \prime} \eta^{k}, \quad\left(\left.\widetilde{\mathscr{B}}_{k}^{(0) \prime} \equiv(k !)^{-1}\left(\frac{\partial}{\partial \eta}\right)^{k}\left\{\widetilde{\mathscr{B}}^{(0)^{\prime}}\right\}\right|_{\eta=0}\right), \hat{\mathscr{M}}^{(0)} \equiv \eta-\left(\tilde{\mathscr{A}}_{0}^{(0)}\right)^{-1} \widetilde{\mathscr{A}}^{(0)}$, $\widetilde{\mathscr{B}}^{(0)} \equiv \sum_{k=0}^{\tilde{r}_{0}} \widetilde{\mathscr{B}}_{k}^{(0) \prime}\left(\tilde{\mathscr{M}}^{(0)}\right)^{k}, \widetilde{\mathscr{I}}^{(1)} \equiv \widetilde{\mathscr{A}}^{(0)}+\widetilde{\mathscr{A}}^{(2)},\left(\widetilde{\mathscr{A}}^{(2)} \equiv\left\{a_{i j}^{(2)}\right\}\right.$ such that $a_{i j}^{(2)} \equiv$ $\left(r_{j}-r_{0}\right) \frac{\partial^{2}}{\partial y \partial \eta}\left\{\hat{a}_{i j}\right\}+\left.\hat{a}_{i j}\right|_{(\eta, \xi, \lambda)=0}$ if $r_{j}+s_{i}=1, a_{i j}^{(2)} \equiv\left(r_{j}-r_{0}+1\right) \frac{\partial}{\partial y}\left\{a_{i j}\right\}$ if $r_{j}+s_{i}=0$ and $a_{i j}^{(2)} \equiv 0$ otherwise, $)$ and $\tilde{\mathscr{M}}^{(1)} \equiv \eta-\left(\widetilde{\mathscr{A}}_{0}^{(0)}\right)^{-1} \widetilde{\mathscr{A}}^{(1)}$. Let us set as $\tilde{M}^{(2)} \equiv \tilde{M}-\tilde{M}^{(0)}, \quad \tilde{M}_{0}^{(1)} \equiv \mathbb{I}, \quad \tilde{M}_{k}^{(1)} \equiv\left[\frac{\partial}{\partial y}, \tilde{M}_{k-1}^{(1)}\right]+\tilde{M}_{k-1}^{(1)} \tilde{M}^{(1)}$ $(k=1,2, \ldots), \widetilde{B}^{(1)} \equiv \sum_{k=0}^{\tilde{r}_{0}} \widetilde{B}_{k}^{\prime} \widetilde{M}_{k}^{(1)}, \widetilde{B}^{(2)} \equiv \widetilde{B}^{(1)}-\widetilde{B}^{(0)}$ and $\widetilde{B}^{(3)} \equiv \widetilde{B}-\widetilde{B}^{(1)}$.

Lemma 6.4. $\left(\left(\tilde{A}_{0}\right)^{-1} \tilde{A}, \widetilde{B}\right)$ is a type of first order pseudo-differential system with which we dealt in the section 5. In fact, $\left(\tilde{A}_{0}\right)^{-1} \tilde{A}=\frac{\partial}{\partial y}$ $-\tilde{M}=\frac{\partial}{\partial y}-\tilde{M}^{(0)}-\tilde{M}^{(2)} . \quad \widetilde{B}=\widetilde{B}^{(0)}+\widetilde{B}^{(2)}+\widetilde{B}^{(3)} . \quad\left(\tilde{\mathscr{M}}^{(0)}, \widetilde{\mathscr{B}}^{(0)}\right)$ the symbol of $\left(\tilde{M}^{(0)}, \widetilde{B}^{(0)}\right)$ that is the principal part of $(\tilde{M}, \widetilde{B})$ are homogeneous functions in $(\xi, \lambda)$ with order 1 and $0 . \quad\left(\eta-\tilde{\mathscr{M}}^{(0)}, \widetilde{\mathscr{B}}^{(0)}\right)$ satisfies <Assumption $(A)>$ and $C_{3}(\lambda) \equiv\left(\begin{array}{cc}-\tilde{M}^{(2)}, & 0 \\ \widetilde{B}^{(3)}, & \widetilde{B}^{(2)}\end{array}\right)$ satisfies (5.2) and (5.12).

Proof. It is easy to show that <Assumption (A)> is satisfied. In fact, $\Gamma_{-}$and $D$ in the section 2 that we obtain if we replace $M$ and $B$ with $-\tilde{\mathscr{M}}^{(0)}$ and $\widetilde{\mathscr{B}}^{(0)}$ are equal to $\mathscr{P}$ and $\mathscr{D}$ in the section 1 (1.5), because $\mathscr{N}_{i}^{\prime}=\left(\mathscr{N}_{i}\right)^{-1}(i=0,1), I=\mathscr{N}_{2}=\mathscr{N}_{3}$ if $|\zeta|=|(\xi, \lambda)|=1,\left(\eta-\tilde{\mathscr{M}}^{(0)}\right)^{-1}$ $=\left(\widetilde{\mathscr{A}}^{(0)}\right)^{-1} \hat{\mathscr{A}}_{0}^{(0)}=\left(\mathscr{N}_{0}\right)^{-1}\left(\hat{\mathscr{A}}^{(0)}\right)^{-1}\left(\mathscr{N}_{1}\right)^{-1} \hat{\mathscr{A}}_{0}^{(0)}$ and $D=\int_{\Gamma} \mathscr{B}^{(0)}\left(\eta-\tilde{\mathscr{M}}^{(0)}\right)^{-1}$ $\mathscr{d} \eta=\int_{\Gamma} \widetilde{\mathscr{B}}^{(0)^{\prime}}\left(\eta-\tilde{\mathscr{M}}^{(0)}\right)^{-1} d \eta=\int_{\Gamma} \mathscr{N}_{2} \hat{\mathscr{B}}^{(0)}\left(\hat{\mathscr{A}}^{(0)}\right)^{-1}\left(\mathscr{N}_{1}\right)^{-1} \hat{\mathscr{A}}_{0}^{(0)} d \eta=\mathscr{D} . \quad W e$ use the remark with respect to $C_{3}(\lambda)$ at the last part of the section 5 . Each term of $M_{2}(\lambda)$ consists of finite sum of the pseudo-differential operators

$$
a_{0}(x, y)\left(\Lambda^{1}(\lambda)-i \partial_{y}\right)^{-\alpha} \Lambda^{\beta}(\lambda) a_{1}^{\prime}\left(x, y, \partial_{x}, \lambda\right)
$$

such that $a_{1}^{\prime}\left(x, y, \partial_{x}, \lambda\right)=a_{1}(x, y) \Lambda^{\gamma}(\lambda), a_{1}(x, y) \partial_{x_{i}} \Lambda^{\gamma-1}(\lambda), a_{1}(x, y) \lambda \Lambda^{\gamma-1}(\lambda)$, $\left[a_{1}(x, y), \Lambda^{1}(\lambda)\right] \partial_{x_{i}} \Lambda^{\gamma-1}(\lambda)$ or $\left[a_{1}(x, y), \Lambda^{1}(\lambda)\right] \Lambda^{\gamma-1}(\lambda)$, where $\alpha \geqq \beta+\gamma$, $\alpha \geqq 0$ and $a_{i}(i=0,1)$ are $C^{\infty}$-functions in $(x, y)$. Thus, $M_{2}(\lambda)$ satisfies the types of estimates (5.13), (5.14) and (5.15), because $\left(\Lambda^{1}(\lambda)-i \partial_{y}\right)^{-\alpha} \Lambda^{\alpha}(\lambda)$ and $\Lambda^{-\alpha+\beta}(\lambda) a_{1}^{\prime}\left(x, y, \partial_{x}, \lambda\right)$ satisfy them. (Refer Lemma 3.19.) Each term of $\Lambda^{-1}(\lambda) B_{2}(\lambda)$ consists of finite compositions of types (6.2) such that $\alpha=0$, and each term of $\Lambda^{-1}(\lambda) B_{3}(\lambda)$ consists of finite sum of finite products $\prod_{\text {finite }} P_{i}$ such that $P_{i}$ are of types (6.2) and one of them has 
$\alpha \geqq 1$, because we have divided $B(\lambda)$ as they are. So $B_{2}(\lambda)$ and $B_{3}(\lambda)$ defined for $C_{0}^{\infty}$-functions in $x$ and in $(x, y)$, respectively, satisfy (5.13) and (5.14).

End of proof.

Lemma 6.5. There exists a constant $\lambda_{0}$ such that for any $\lambda \geqq \lambda_{0}$ the partial differential system $(\hat{A}, \hat{B})$ defined from $H_{m}^{\infty}\left(R_{+}^{n+1}\right)$ to $H_{m}^{\infty}\left(R_{+}^{n+1}\right) \times H_{l}^{\infty}\left(R^{n}\right) \quad\left(\hat{A}\right.$ defined on $H_{m}^{\infty}\left(R^{n+1}\right)$ if $\left.\Omega^{v} \cap \partial \Omega=\phi\right)$ has the inverse $\hat{R}(\lambda)$, which satisfies that for $F=\left(F_{1}, F_{2}\right) \in H_{m}^{\infty}\left(R_{+}^{n+1}\right) \times H_{l}^{\infty}\left(R^{n}\right)$ and for $s \geqq \tilde{r}_{0}$

$$
\begin{aligned}
& \left\{\left\|\left(\Lambda^{1}(\lambda)-i \partial_{y}\right)^{s} \cdot N_{0}^{-1} \cdot \hat{R}(\lambda) F\right\|_{+}^{2}+\left\|\left.\Lambda^{\alpha-1 / 2}(\lambda) \cdot N_{3}^{-1} \hat{R}(\lambda) F\right|_{y=0}\right\|^{2}\right\}^{1 / 2} \\
& \leqq c_{s} \lambda^{-1}\left\{\left\|\left(\Lambda^{1}(\lambda)-i \partial_{y}\right) N_{1} \cdot N_{1}\right\|_{+}^{2}+\left\|\Lambda^{s+1 / 2}(\lambda) \cdot N_{2} F_{2}\right\|^{2}\right\}^{1 / 2} . \\
& \left(\left\|\left(\Lambda^{1}(\lambda)-i \partial_{y}\right)^{s} \cdot N_{0}^{-1} \cdot \hat{R}(\lambda) F\right\| \leqq c_{s} \lambda^{-1}\left\|\left(\Lambda^{1}(\lambda)-i \partial_{y}\right)^{s} \cdot N_{1} F\right\|\right. \\
& \text { if } \left.\Omega^{v} \cap \partial \Omega=\phi .\right)
\end{aligned}
$$

Remark. We always identify a distribution $u \in \mathscr{D}^{\prime}(\Omega)$ and a pair of distributions $(u, v) \in \mathscr{D}^{\prime}(\Omega) \times \mathscr{D}^{\prime}(\partial \Omega)$ if $u^{0}=\left.u\right|_{\partial \Omega}$ the boundary value of $u$ is well defined and if $u^{0}=v$.

Proof. Theorems $6 \sim 9$ are valid for $\left(\left(\tilde{A}_{0}\right)^{-1} \cdot \tilde{A}, \widetilde{B}\right)$ by Lemma 6.4. So, there exists the inverse $\widetilde{R}_{1}(\lambda)$ of $\left(\left(\tilde{A}_{0}\right)^{-1} \cdot \widetilde{A}, \widetilde{B}\right)$. Corollary 2 of Theorem 9 and Definition 6.1 show that

$$
\tilde{R}_{1}^{\prime}(\lambda)=\tilde{R}_{1}(\lambda) \cdot\left(\begin{array}{ll}
\left(\tilde{A}_{0}\right)^{-1} & , 0 \\
-\widetilde{B}^{\prime \prime} \cdot\left(\tilde{A}_{0}\right)^{-1}, I
\end{array}\right)
$$

gives the inverse of $(\tilde{A}, \widetilde{B})$ and it satisfies the same estimate of Theorems $7 \sim 9$ if $s \geqq \tilde{r}_{0}$ because $\Lambda^{1}(\lambda) \cdot \widetilde{B}^{\prime \prime}$ consists of the composition of the types (6.2). (If $\Omega^{v} \cap \partial \Omega=\phi, \widetilde{R}_{1}^{\prime}(\lambda)=\widetilde{R}_{1}(\lambda) \cdot\left(\widetilde{A}_{0}\right)^{-1}$. We omit the proof of existence of $\widetilde{R}_{1}(\lambda)$. Refer Definition 5.5 and Lemmas 5.5 and 5.8.) We set

$$
\hat{R}(\lambda)=N_{0} \cdot \tilde{R}_{1}^{\prime}(\lambda) \cdot\left(\begin{array}{cc}
N_{1}, & 0 \\
0, N_{2}
\end{array}\right) .
$$

(If $\Omega^{v} \cap \partial \Omega=\phi$, we set $\hat{R}(\lambda)=N_{0} \cdot \widetilde{R}_{1}^{\prime}(\lambda) \cdot N_{1}$.) This is what we want。 
It satisfies the estimate of this lemma. In fact, if $u=\widehat{R}(\lambda) F$,

$$
\begin{aligned}
& \left\|\left(\Lambda^{1}(\lambda)-i \partial_{y}\right)^{s} \cdot N_{0}^{-1} u\right\|_{+} \\
& =\left\|\left(\Lambda^{1}(\lambda)-i \partial_{y}\right)^{s} \cdot \widetilde{R}_{1}^{\prime}(\lambda) \cdot\left(\begin{array}{cc}
N_{1}, & 0 \\
0, N_{2}
\end{array}\right) F\right\|_{+} \\
& \leqq c_{s} \lambda^{-1}\left\{\left\|\left(\Lambda^{1}(\lambda)-i \partial_{y}\right)^{\alpha} \cdot N_{1} F_{1}\right\|_{+}^{2}+\left\|\Lambda^{s+1 / 2}(\lambda) \cdot N_{2} F_{2}\right\|^{2}\right\}^{1 / 2}
\end{aligned}
$$

and

$$
\left\|\left.\Lambda^{s-1 / 2}(\lambda) \cdot N_{3}^{-1} u\right|_{y=0}\right\| \leqq c\left\|\left(\Lambda^{1}(\lambda)-i \partial_{y}\right)^{s} \cdot N_{0}^{-1} u\right\|_{+}
$$

if $s>1 / 2$.

End of proof.

Definition 6.2. Let us define Hilbert space $\Theta_{i}^{s}(\lambda, \Omega)(i=0,1)$ as followings.

$$
\Theta_{0}^{s}(\lambda, \Omega) \equiv \prod_{j=1}^{m} H^{r_{0}-r_{j}+s}(\Omega) \times \prod_{j=1}^{m} H^{r_{0}-r_{j}+s-1 / 2}(\partial \Omega)
$$

with the norm $\theta_{0}(U) \equiv\left\{\sum_{v} \rho_{0 v}(U)^{2}\right\}^{1 / 2}$;

$$
\rho_{0 v}(U) \equiv\left\{\left\|\left(\Lambda^{1}(\lambda)-i \partial_{y}\right)^{s} \cdot N_{0}^{-1} \beta_{v} u\right\|_{+}^{2}+\left\|\Lambda^{s-1 / 2}(\lambda) N_{3}^{-1} \beta_{v}^{0} v\right\|^{2}\right\}^{1 / 2}
$$

for $U=(u, v) \in H_{m}^{\infty}(\Omega) \times H_{m}^{\infty}(\partial \Omega)$.

$$
\Theta_{1}^{s}(\lambda, \Omega) \equiv \prod_{j=1}^{m} H^{1-r_{0}-s_{j}+s}(\Omega) \times \prod_{j=1}^{l} H^{-r_{0}-t_{j}+s+1 / 2}(\partial \Omega)
$$

with the norm $\theta_{1}(U) \equiv\left\{\sum_{v} \rho_{1 v}(U)^{2}\right\}^{1 / 2}$

$$
\rho_{1 v}(U) \equiv\left\{\left\|\left(\Lambda^{1}(\lambda)-i \partial_{y}\right)^{s} \cdot N_{1} \beta_{v} u\right\|_{+}^{2}+\left\|\Lambda^{s+1 / 2}(\lambda) \cdot N_{2} \beta_{v}^{0} v\right\|^{2}\right\}^{1 / 2}
$$

for $U=(u, v) \in H_{m}^{\infty}(\Omega) \times H_{l}^{\infty}(\partial \Omega)$.

Here, $\left\{\beta_{v}\right\}$ is the system of $C^{\infty}$-functions in Lemma 6.1, $\beta_{v}^{0}=\left.\beta_{v}\right|_{\partial \Omega}$ and $\left(\Lambda^{1}(\lambda)-i \partial_{y}\right)^{s}, \Lambda^{s}(\lambda), N_{i},\|\cdot\|_{+}$and $\|\cdot\|$ are defined with respect to the local coordinate on $\Omega^{v}$.

Let us denote $\alpha_{v} U \equiv\left(\alpha_{v} u, \alpha_{v}^{0} v\right)$ for $U=(u, v) \in \Theta_{i}^{\mathrm{s}}(\lambda, \Omega)$. We consider

$$
R^{\prime}(\lambda) \equiv \sum_{v} \alpha_{v} \hat{R}_{v}(\lambda) \alpha_{v}
$$


which gives a good approximation for the inverse of $\left(\begin{array}{c}A, 0 \\ 0, B\end{array}\right)$. In fact

$$
\begin{aligned}
& R^{\prime}(\lambda)\left(\begin{array}{c}
A, 0 \\
0, B
\end{array}\right)=\sum_{v} \alpha_{v} \hat{R}_{v}(\lambda) \alpha_{v}\left(\begin{array}{c}
\hat{A}_{v}, \\
0 \\
0, \hat{B}_{v}
\end{array}\right)
\end{aligned}
$$

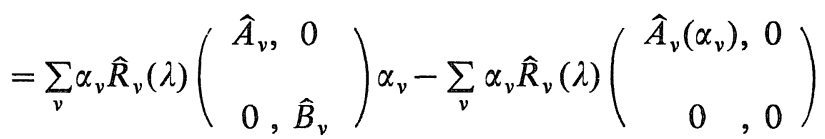

$$
\begin{aligned}
& =\sum_{v} \alpha_{v}^{2}-\sum_{v} \alpha_{v} \hat{R}_{v}(\lambda) A\left(\alpha_{v}\right) \\
& =I-\sum_{v} \alpha_{v} \hat{R}_{v}(\lambda) A\left(\alpha_{v}\right)
\end{aligned}
$$

and

$$
\begin{aligned}
& \left(\begin{array}{c}
A, 0 \\
0, B
\end{array}\right) R^{\prime}(\lambda)=\sum_{v}\left(\begin{array}{cc}
\hat{A}_{v}, & 0 \\
0, \hat{B}_{v}
\end{array}\right) \alpha_{v} \hat{R}_{v}(\lambda) \alpha_{v}
\end{aligned}
$$

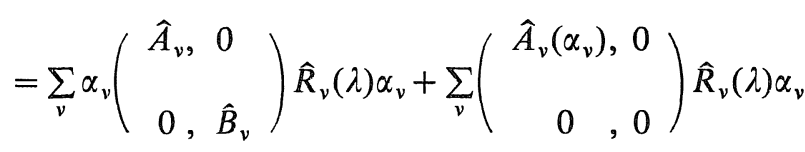

$$
\begin{aligned}
& =\sum_{v} \alpha_{v}^{2}+\sum_{v} A\left(\alpha_{v}\right) \hat{R}_{v}(\lambda) \alpha_{v} \\
& =I+\sum_{v} A\left(\alpha_{v}\right) \hat{R}_{v}(\lambda) \alpha_{v} .
\end{aligned}
$$

Here, $A\left(\alpha_{v}\right)$ stands for $\left(\begin{array}{c}A\left(\alpha_{v}\right), 0 \\ 0,0\end{array}\right)$.

We show that $I-\sum_{v} \alpha_{v} \hat{R}_{v}(\lambda) A\left(\alpha_{v}\right)$ and $I+\sum_{v} A\left(\alpha_{v}\right) \hat{R}_{v}(\lambda) \alpha_{v}$ are invertible on $\Theta_{0}^{s}(\lambda, \Omega)$ and $\Theta_{1}^{s}(\lambda, \Omega)$ for $\lambda \geqq \lambda_{0}$ and $s \geqq \tilde{r}_{0}$, respectively.

Lemma 6.6. Let us put $T_{0} \equiv \sum_{v} \alpha_{v} \hat{R}_{v}(\lambda) A\left(\alpha_{v}\right)$ and $T_{1} \equiv \sum_{v} A\left(\alpha_{v}\right) \hat{R}_{v}(\lambda) \alpha_{v}$. Then there exists $\lambda_{0}$ such that for $\lambda \geqq \lambda_{0}$,

$$
\begin{aligned}
& \theta_{0}\left(R^{\prime}(\lambda) F\right) \leqq c_{s} \lambda^{-1} \theta_{1}(F) \\
& \theta_{0}\left(T_{0} F_{0}\right) \leqq c_{s} \lambda^{-1} \theta_{0}\left(F_{0}\right) \\
& \theta_{1}\left(T_{1} F_{1}\right) \leqq c_{s} \lambda^{-1} \theta_{1}\left(F_{1}\right) .
\end{aligned}
$$


Proof. Let us put $V_{0} \equiv \hat{R}_{v}(\lambda) A\left(\alpha_{v}\right) F_{0}$ and $V_{1} \equiv \hat{R}_{v}(\lambda) \alpha_{v} F_{1}$. If $\Omega^{v} \cap$ $\partial \Omega \neq \phi$, then by Corollary 2 of Theorem 9 ,

$$
\begin{aligned}
& \rho_{0 \mu}\left(\alpha_{v} V_{1}\right) \\
& \quad \leqq c_{s v} \rho_{0 v}\left(V_{1}\right) \quad \text { because } \beta_{v} \beta_{\mu} \alpha_{v}=\beta_{v} \alpha_{v} \\
& \quad \leqq c_{s v}^{\prime} \lambda^{-1} \rho_{1 v}\left(\alpha_{v} F_{1}\right) \quad \text { because of Theorem } 9 \\
& \quad \leqq c_{s v}^{\prime \prime} \lambda^{-1} \rho_{1 v}\left(F_{1}\right), \\
& \rho_{0 \mu}\left(\alpha_{v} V_{0}\right) \\
& \quad \leqq c_{s v} \rho_{0 v}\left(V_{0}\right) \quad \text { because } \beta_{v} \beta_{\mu} \alpha_{v}=\beta_{v} \alpha_{v} \\
& \quad \leqq c_{s v}^{\prime} \lambda^{-1} \rho_{1 v}\left(A\left(\alpha_{0}\right) F_{0}\right) \quad \text { because of Theorem } 9 \\
& \quad \leqq c_{s v}^{\prime \prime} \lambda^{-1} \rho_{0 v}\left(F_{0}\right) \quad \text { because } \\
& \left\|\left(\Lambda^{1}(\lambda)-i \partial_{y}\right)^{s} \cdot N_{0}^{-1} A\left(\alpha_{0}\right) u\right\| \leqq c\left\|\left(\Lambda^{1}(\lambda)-i \partial_{y}\right)^{s} \cdot N_{1} u\right\|, \\
& \rho_{1 \mu}\left(A\left(\alpha_{v}\right) V_{1}\right) \\
& \quad \leqq c_{s v} \rho_{1 v}\left(A\left(\alpha_{v}\right) V_{1}\right) \quad \text { because } \beta_{v} \beta_{\mu} A\left(\alpha_{v}\right)=\beta_{\mu} A\left(\alpha_{v}\right) \\
& \quad \leqq c_{s v}^{\prime} \rho_{0 v}\left(V_{1}\right) \quad \text { because of }(6.8) \\
& \quad \leqq c_{s v}^{\prime \prime} \lambda^{-1} \rho_{1 v}\left(\alpha_{v} F_{1}\right) \quad \text { because of Theorem } 9 \\
& \leqq c_{s}^{\prime \prime \prime} \lambda^{-1} \rho_{1 v}\left(F_{1}\right) .
\end{aligned}
$$

Summing up these in $\mu$ and $v$ we obtain Lemma 6.6. End of proof.

Lemma 6.7. There exists $\lambda_{s}$ for $s \geqq \tilde{r}_{0}$ such that for $\lambda \geqq \lambda_{s} I-T_{0}$ and $I+T_{1}$ are isomorphisms on $\Theta_{1}^{s}(\lambda, \Omega)$ and $\Theta_{0}^{s}(\lambda, \Omega)$, respectively. So, $\left(I-T_{0}\right)^{-1} R^{\prime}(\lambda)$ and $R^{\prime}(\lambda)\left(I+T_{1}\right)^{-1}$ are left and right inverses of the minimal closed extension of $\left(\begin{array}{c}A, 0 \\ 0, B\end{array}\right)$ with the definition domain $H_{m}^{\infty}(\Omega)$ from $\Theta_{0}^{s}(\lambda, \Omega)$ to $\Theta_{1}^{s}(\lambda, \Omega)$. Therefore they are same.

Proof. This lemma follows immediately from Lemma 6.6.

End of proof. 
This lemma means (1.1) has the unique solution in $\Theta_{0}^{s}(\lambda, \Omega)$ for data given in $\Theta_{1}^{s}(\lambda, \Omega)$. So we complete the proof of Theorem 1 if we show this solution belongs to $H_{m}^{\infty}(\Omega)$ when the data is given in $H_{m}^{\infty}(\Omega) \times H_{l}^{\infty}(\partial \Omega)$. We shall show it locally on $\bar{\Omega}$. It is clear at the inner point of $\Omega$. So, we can assume $\Omega=R_{+}^{n+1}$ because, if $U \in \Theta_{0}^{\mathrm{s}}(\lambda, \Omega)$ is a solution of (1.1) for a datum $F$ given in $\Theta_{1}^{s}(\lambda, \Omega), \varphi U \in \Theta_{0}^{s}(\lambda, \Omega)$ is a solution of (1.1) for a datum $F-A(\varphi) U \in \Theta_{1}^{s}(\lambda, \Omega)$, where $\varphi \in C_{0}^{\infty}(\bar{\Omega})$. In Lemma 6.5, $(\widehat{A}, \hat{B})$ has the inverse $\hat{R}(\lambda)$ for $\lambda \geqq \lambda_{0}$. Corollary 2 of Theorem 9 shows $\hat{R}(\lambda)$ is also a bounded operator from $\Theta_{1}^{s}\left(\lambda, R_{+}^{n+1}\right)$ to $\Theta_{0}^{s}\left(\lambda, R_{+}^{n+1}\right)$ for $\lambda \geqq \lambda_{0}$.

Lemma 6.8. If $s \geqq \tilde{r}_{0}, F \in \Theta_{1}^{s}\left(\lambda, R_{+}^{n+1}\right)$ and $\psi F \in \Theta_{1}^{s+1 / 2}\left(\lambda, R_{+}^{n+1}\right)$, then $\hat{R}(\lambda) F \in \Theta_{0}^{s}\left(\lambda, R_{+}^{n+1}\right)$ and $\psi \hat{R}(\lambda) F \in \Theta_{0}^{s+1 / 2}\left(\lambda, R_{+}^{n+1}\right)$ for $\lambda \geqq \lambda_{0}\left(\lambda_{0}\right.$ does not depend on s), where $\psi \in C_{0}^{\infty}\left(\overline{R_{+}^{n+1}}\right), \psi^{0}=\left.\psi\right|_{y=0}, F=\left(f_{1}, f_{2}\right)$ and $\psi F=\left(\psi f_{1}\right.$, $\left.\psi^{0} f_{2}\right)$.

Proof. Let us put $G_{0}=\left(\begin{array}{c}N_{1}^{-1}, \\ 0 \\ 0, N_{2}^{-1}\end{array}\right) F$. Then $G_{0} \in H^{s}\left(R_{+}^{n+1}\right) \times$ $H^{s+1 / 2}\left(R^{n}\right)$ and $\psi G_{0} \in H^{s+1 / 2}\left(R_{+}^{n+1}\right) \times H^{s+1}\left(R^{n}\right)$. (Refer Lemma 3.19 and Definition 6.2.) Thus,

$$
G_{1}=\left(\begin{array}{cc}
\left(\tilde{A}_{0}\right)^{-1} & , 0 \\
-\widetilde{B}^{\prime \prime}\left(\tilde{A}_{0}\right)^{-1}, I
\end{array}\right) G_{0} \in H^{s}\left(R_{+}^{n+1}\right) \times H^{s+1 / 2}\left(R^{n}\right)
$$

and

$$
\psi G_{1} \in H^{s+1 / 2}\left(R_{+}^{n+1}\right) \times H^{s+1}\left(R^{n}\right) .
$$

(Refer Definition 6.1 for $\widetilde{B^{\prime \prime}}$.) By Corollary 1 of Theorem 9

$$
U_{0}=\widetilde{R}_{1}(\lambda) G_{1} \in H^{s}\left(R_{+}^{n+1}\right) \times H^{s-1 / 2}\left(R^{n}\right)
$$

and $\psi U_{0} \in H^{s+1 / 2}\left(R_{+}^{n+1}\right) \times H^{s}\left(R^{n}\right)$. Since $U=\widehat{R}(\lambda) F=N_{0} U_{0}$, we obtain $U \in \Theta_{0}^{s}\left(\lambda, R_{+}^{n+1}\right)$ and $\psi U \in \Theta_{0}^{s+1 / 2}\left(\lambda, R_{+}^{n+1}\right)$ End of proof.

Lemma 6.8 means that, if $F \in H_{m}^{\infty}\left(\Omega_{0}\right) \times H_{l}^{\infty}\left(\Omega_{0} \cap R^{n}\right) \cap \Theta_{1}^{s}\left(\lambda, R_{+}^{n+1}\right)$, $u=\hat{R}(\lambda) F \in H_{m}^{\infty}(K)$ for any compact set of $K \Omega_{0}$ which is an open set in $\overline{R_{+}^{n+1}}$. Thus the equation (1.1) has the unique solution $u$ in $\Theta_{0}^{s}(\lambda, \Omega)$ for data $F=(f, g)$ in $\Theta_{1}^{s}(\lambda, \Omega)$ if $\lambda \geqq \lambda_{0}$ and $s \geqq \tilde{r}_{0}$, and 
$\operatorname{sing} \operatorname{supp} u=\operatorname{sing} \operatorname{supp} F=\operatorname{sing} \operatorname{supp} f \cup \operatorname{sing} \operatorname{supp} g \subset \bar{\Omega}$.

We complete the proof of Theorem 1 .

\section{§7. Remarks}

1. $(A, B)$ is properly linked by $\lambda$ if $(A, B)$ is strongly linked by $\lambda$. $(A, B)$ is strongly linked by $\lambda$ if and only if $(A, B)$ is linked by $\lambda$ and coercive.

2. At the example in the section 1 we can verify it is properly linked by $\lambda$. It is finally enough to make it sure for $\lambda \psi^{-1}+\varphi$ to satisfy the conditions of Assumption (A), where $\psi=\left(a_{i j} \xi_{i} \xi_{j}+\lambda^{2}\right)^{1 / 2}(\neq 0$ if $\zeta=(\xi, \lambda)$ $\neq 0)$. We use the well known property that $|\operatorname{grad} \varphi|^{2} \leqq 2|\varphi| \sup \mid$ hess $\varphi \mid$ if $\varphi \geqq 0$. Then, it is clear that $\left|\lambda \psi^{-1}+\varphi\right| \geqq c \lambda|\zeta|^{-1},\left|\operatorname{grad}_{x}\left(\lambda \psi^{-1}+\varphi\right)\right| \leqq$ $\left|\lambda \psi^{-2} \operatorname{grad}_{x} \psi\right|+\left|\operatorname{grad}_{x} \varphi\right| \leqq c\left(\left|\lambda \psi^{-1}\right|+|\varphi|^{1 / 2}\right) \leqq c\left|\lambda \psi^{-1}+\varphi\right|^{1 / 2}$ and $\mid \operatorname{grad}_{\xi}\left(\lambda \psi^{-1}\right.$ $+\varphi)|=| \lambda \psi^{-2} \operatorname{grad}_{\xi} \psi|\leqq c| \lambda \psi^{-1}+\left.\varphi|| \zeta\right|^{-1}$.

We give next a more general type of boundary conditions. Let $\Omega$ be $R_{+}^{n+1}, A=\left(\frac{\partial}{\partial y}\right)^{2}+\sum_{1 \leqq i, j \leqq n} a_{i j} \frac{\partial^{2}}{\partial x_{i} \partial x_{j}}-a_{n+1} \lambda^{2}+d_{1}$ be a second order elliptic operator with real coefficients and $B=b_{0} \frac{\partial}{\partial y}+\sum_{i=1}^{n} b_{i} \frac{\partial}{\partial x_{i}}+b_{n+1} \lambda+d_{2}$ be a boundary operator with real coefficients, where $d_{1}$ and $d_{2}$ are lower order terms. We put $\sigma=i\left(\sum a_{i j} \xi_{i} \xi_{j}+a_{n+1} \lambda^{2}\right)^{1 / 2}, \zeta=(\xi, \lambda)$ and $\alpha=$ the minimum of $\sigma(\zeta)$ on $\sum_{i=1}^{n} b_{i} \xi_{i}=0$. If we assume that

$$
\begin{aligned}
& b_{0} \leqq 0, b_{0} \alpha>b_{n+1},\left|b_{i}\right|^{2} \leqq c b_{0} \quad i=1, \ldots, n \text { and } \\
& \left|\operatorname{grad}_{x}\left(\sum_{k=1}^{n} b_{k} \xi_{k}\right)\right|^{2} \leqq c\left(b_{0}+\left|\sum_{k=1}^{n} b_{k} \xi_{k}\right|\right) \quad \text { on } \quad|\xi|=1,
\end{aligned}
$$

then we have same results as for the example. The last inequality is satisfied if $\left|\operatorname{grad}_{x} b_{k}\right|^{2} \leqq c b_{0}$ or if $\left(b_{k}\right)=b\left(\hat{b}_{k}\right)$ such that $\sum\left|\hat{b}_{k}\right|^{2}=1$ and $\left|\operatorname{grad}_{x} b\right|^{2} \leqq c\left(b_{0}+|b|\right)$.

3. In this paper we have considered the boundary value problem. The discussion in the section 4 shows us the way to give a sufficient condition for solvability and hypoellipticity of non-elliptic pseudo-differential operators if we watch pseudo-differential operators only on the 
boundary $R^{n}$ of $\overline{R_{+}^{n+1}}$. Let us consider $M(x, \zeta) m \times m$ matrix of infinitely differentiable functions on $R^{n} \times\left\{\overline{R_{+}^{n+1}}-(0,0)\right\}$ which independent of $x$ outside a bounded set of $R^{n}$ and a homogeneous function of order 1 in $\zeta$. We assume that $M(x, \zeta)$ satisfies $\lambda|h| \leqq c|M(x, \zeta) h|, \mid \operatorname{grad}_{(x, \xi)} M(x$, $\zeta)\left.h\right|^{2} \leqq c|M(x, \zeta) h|$ and $\left|\operatorname{grad}_{(x, \xi)} M^{*}(x, \zeta) h\right|^{2} \leqq c\left|M^{*}(x, \zeta) h\right|$ for $h \in \mathbb{C}^{m}, \zeta$ $=(\xi, \lambda),|h|=1$ and $|\zeta|=1$. Then, the equation $M(x, \partial, \lambda) u=f$ on $R^{n}$ has the inverse $R(\lambda)$ on $H^{-\infty}\left(R^{n}\right), R(\lambda) u \in H(s, k, \chi)$ if $f \in H(s, k, \chi)$, and it holds that $\rho_{k}(R(\lambda) f) \leqq c_{s k} \lambda^{-1} \rho_{k}(f)+c_{s h}(\chi) \lambda^{-3 / 2} \rho_{k-1}(f)$, where $\chi$ is a system of infinitely differentiable functions such that $\chi=\left\{\chi_{j}, j=0,1,2, \ldots\right.$; $\chi_{0} \equiv 1, \chi_{j} \equiv 1$ or $\in C_{0}^{\infty}\left(R^{n}\right)$, and $\left.\chi_{j} \chi_{j+1}=\chi_{j+1}\right\}$, and $H(s, k, \chi)$ stands for the completion of $C_{0}^{\infty}\left(R^{n}\right)$ by the norm $\rho_{h}(f) \equiv\left(\sum_{j=0}^{k}\left\|\Lambda^{s+j / 2}(\lambda) \chi_{j} f\right\|^{2}\right)^{1 / 2}$. Moreover if $M_{1}(x, \partial, \lambda)$ is a composition of pseudo-differential operator which satisfies $\rho_{h}\left(M_{1}(x, \partial, \lambda) f\right) \leqq c_{s k} \rho_{h}(f)+c_{s h}(\chi) \lambda^{-1 / 2} \rho_{k-1}(f)$. Then, we obtain the same result for $\left\{M(x, \partial, \lambda)+M_{1}(x, \partial, \lambda)\right\} u=f$.

4. There exists a constant $\lambda_{0}$ such that $\lambda^{2}-\varphi^{2} \Delta$ is solvable and hypoelliptic for $\lambda \geqq \lambda_{0}$ if $\varphi$ is a non-negative infinitely differentiable function. $\lambda-i \psi \Lambda(\lambda)+i[\Lambda(\lambda), \psi]$ and $\lambda+i \psi \Lambda(\lambda)$ satisfy the conditions of the previous remark 3 and so its results, where $\psi=\varphi\left(1+\varphi^{2}\right)^{-1 / 2}$. We consider the product of them. $\left(1-\psi^{2}\right)^{-1}(\lambda-i \psi \Lambda(\lambda)+i[\Lambda(\lambda), \psi])(\lambda+i \psi \Lambda(\lambda))=\lambda^{2}$ $-\varphi^{2} \Delta+N$ because $\Lambda^{2}(\lambda)=\lambda^{2}-\Delta$. We have also the same results for this operator and so for $\lambda^{2}-\varphi^{2} \Delta$ since $N$ is a negligible term.

5. We give an example of non-hypoelliptic differential operator. We construct it by the following procedure.

$$
\begin{aligned}
& \tilde{\psi}(i, j) \equiv\left\{\begin{array}{l}
1,(a-1 / 8) \pi \leqq x \leqq(a+1 / 8) \pi ; a=2^{i+1}-1+j \\
0, \text { otherwise }
\end{array}\right. \\
& \sigma ; \sigma \geqq 0, \int \sigma d x=1 \text { and } \operatorname{supp} \sigma \subset(-\pi / 16, \pi / 16) \\
& \psi(i, j) \equiv \sigma * \tilde{\psi}(i, j) \\
& \psi \quad \equiv \sum_{\substack{1 \leqq j \leqq 2 \\
0 \leqq i \infty \infty}}(-1)^{i} 2^{-i} j \psi(i, j)
\end{aligned}
$$




$$
\begin{aligned}
& \theta(v) \equiv \sum_{\substack{i, j) ; v=(-1)^{i} 2-i_{j} \\
1 \leqq j \leqq 2 i \\
0 \leqq i<\infty}} \psi(i, j) \\
& \tilde{f} \equiv e^{-\lambda x}(\sin x)^{\lambda} \log |\sin x| \\
& \tilde{f}(v) \equiv \theta(v) \tilde{f} \\
& \tilde{\varphi} \equiv \sin x(\cos x-\sin x)^{-1} \psi \\
& f(v) \equiv\left\{\begin{array}{cc}
\tilde{f}(v)\left(e^{1 / x}\right), & x>0 \\
0, & x \leqq 0
\end{array}\right. \\
& \varphi \equiv\left\{\begin{array}{cc}
-x^{2-} e^{1 / x} \tilde{\varphi}\left(e^{1 / x}\right), & x>0 \\
0, & x \leqq 0
\end{array}\right. \\
& \tilde{h} \equiv\left(\lambda \psi-\lambda v+\tilde{\varphi} \frac{d}{d x} \theta(v)\right) \tilde{f}+\theta(v) e^{-\lambda x}(\sin x)^{\lambda-1} \\
& h \equiv \begin{cases}h\left(e^{1 / x}\right), & x>0 \\
0, & x \leqq 0 .\end{cases}
\end{aligned}
$$

Then, $\varphi \frac{d}{d x} f(v)=\lambda v f(v)+h . \quad \varphi$ and $h$ are infinitely differentiable but $f(v)$ is not so at the neighborhood of origin. Thus, $\left(\varphi \frac{d}{d x}-\lambda v\right)$ is not hypoelliptic if $\lambda$ is positive integer and $v$ is one of $(-1)^{i} 2^{-i} j$. Using this differential operator we can easily construct the boundary value problem which is linked by $\lambda$ but not properly linked by $\lambda$.

6. Let $A$ be a symmetric system of first order and elliptic. Let $\operatorname{ker} B$ be a maximal dissipative boundary condition with respect to $A$. Then $(A+\lambda, B)$ is linked by $\lambda$. Moreover it satisfies (1.7). But it is not true to be properly linked by $\lambda$.

7. We can give weaker conditions if it is not necessary to permit perturbations as large as at the remark 3. Let $M(x, \zeta)$ be the same one but its homogeneous order in $\zeta$ be 0.1$) M(x, \zeta)$ is invertible if $\lambda>0$ and $|\zeta|=1$. 2) For each $(x, \zeta)(|\zeta|=1$ and $\lambda=0)$ there exist its neighborhood $\Omega(x, \zeta)$ and positive real number $\delta$ and $\varepsilon$ such that $0<\delta<$ 
$\varepsilon, \lambda \leqq c|M(x, \zeta) u|^{\varepsilon}$ and $\left|\partial_{\xi}^{\alpha} \partial_{x}^{\beta} \partial_{\lambda}^{\gamma} M(x, \zeta) u\right| \leqq c|M(x, \zeta) u|^{1-\delta|\beta|-(\varepsilon-\delta)|\alpha|-(2 \varepsilon-\delta) \gamma}$ on $\Omega(x, \zeta) \cap\{\lambda \geqq 0\}$ for all multi-indices $(\alpha, \beta, \gamma)$ satisfying $\delta|\beta|+(\varepsilon-\delta)|\alpha|$ $+(2 \varepsilon-\delta) \gamma<1$ if $u \in \mathbb{C}^{m}$ and $|u|=1$.

If we assume 1) and 2), then we conclude that there exists a positive $\lambda_{0}$ such that $M(x, \partial, \lambda)$ has a solution in $\mathscr{S}$ for any data in $\mathscr{S}$ when $\lambda \geqq \lambda_{0}$. On the other hand if the adjoint symbol $M^{*}(x, \zeta)$ satisfies 1$)$ and 2), then we can find $\lambda_{0}$ such that $\operatorname{sing} \operatorname{supp} M(x, \partial, \lambda) u=\operatorname{sing} \operatorname{supp} u$ for any $u \in \mathscr{S}^{\prime}$ when $\lambda \geqq \lambda_{0}$. Let $\mathscr{C}$ stand for a ring consisting of symbols $C(x, \zeta)$ such that $C(x, \zeta)$ is a $m \times m$ matrix valued infinitely differentiable function on $R^{n} \times\left\{\overline{R_{+}^{n+1}}-(0)\right\}$, which is independent of $x$ outside a bounded set in $R^{n}$, and satisfies $\left|\partial_{\xi}^{\alpha} \partial_{x}^{\beta} C(x, \zeta)\right| \leqq c_{\alpha \beta}|\zeta|^{-|\alpha|}$ for all multiindices $(\alpha, \beta)$. Let $\mathscr{L}$ and $\mathscr{R}$ stand for the left and right ideals of $\mathscr{C}$ generated by $\left\{\partial_{\xi}^{\alpha} \partial_{x}^{\beta} \partial_{\lambda}^{\gamma} M(x, \zeta) ; 1 \leqq|\beta| \leqq|\alpha|+\gamma\right\}$, respectively. For $M(x$, $\partial, \lambda)+C(x, \partial, \lambda)$ we have same results with another constant $\lambda_{0}(C)$ if $C(x, \zeta)$ is an element of $\mathscr{L}$ or $\mathscr{R}$ and if we assume 1) and 2) to its principal part $M(x, \zeta)$ or the adjoint $M^{*}(x, \zeta)$, respectively.

8. We can find out some well known hypoelliptic partial differential operators by using the conditions at the remark $7 . \lambda^{2}-\varphi^{2} \Delta$ we noted at the remark 4 is also proved to be hypoelliptic by the remark 7 . We have a similar example. $\psi^{3} \Delta+i \lambda^{2}$, where $\psi$ is a real valued $C^{\infty}$-function, is also hypoelliptic and solvable for large $\lambda$. We can show that a parabolic equation $\frac{\partial}{\partial t}+a\left(x, \partial_{x}\right)$ is hypoelliptic and solvable, where $a(x$, $\left.\partial_{x}\right)=\sum_{|\alpha|=2 m} a_{\alpha}(x) \partial_{x}^{\alpha}$ is an elliptic operator of order $2 m>0$ with real coefficients. We may assume $a(x, \xi) \geqq 0$. Let $u$ be a solution of the parabolic equation. Consider the equation which is satisfied by $e^{-\lambda t} u(t /$ $\left.\lambda^{2 m-1}, x\right)$. Then, we have $\lambda^{2 m}+\lambda^{2 m-1} \frac{\partial}{\partial t}+a\left(x, \partial_{x}\right)$, which satisfies the conditions at the remark 7 .

9. We have receipt two preprints [15] and [16] closely related to our results. We note here they have given some interest results for second order elliptic equations. 


\section{Reference}

[1] Agmon, S., On the eigenfunctions and eigenvalues of general elliptic boundary value problems, Comm. Pure Appl. Math., 15 (1962), 119-147.

[2] Agmon, S., Douglis, A. and Nirenberg, L., Estimates near the boundary for solutions of elliptic partial differential equations satisfying general boundary conditions II, Comm. Pure Appl. Math., 17 (1964), 35-92.

[3] Boutet de Monvel, L., Boundary problems for pseudo-differential operators, Acta Math., 126 (1971) 11-51.

[4] Calderon, A.P. and Vaillancourt, R., A calss of bounded pseudo-differential operators, Proc. Math. Acad. Sci. USA 69 (1972), 1185-1187.

[5] Egorov, Ju. V., Nondegenerate subelliptic pseudo-differential operators, Mat. Sbornik Tom 82 (124) (1970), No. 3, (Math. USSR Sbornik vol. 11 (1970) No. 3, 291-309).

[6] Grušin, V. V., Hypoclliptic differential equations and pscudo-diffcrential operators with operator-valued symbols, Mat. Sbornik Tom 88 (130) (1972) No. 4, (Math. USSR Sbornik vol. 17 (1972) No. 2, 497-514).

[7] Hörmander, L., Pseudo-differential operators and non elliptic boundary problems, Ann. of Math. 83 (1966) 129-209.

[8] Hörmander, L., Pseudo-differential operators and hypoelliptic equations, Proc. Symp. on singular integrals, Chicago, April 1966, Amer. Math. Soc. 10 (1967) 138-183.

[9] Kohn, J. J. and Nirenberg, L., Non coercive boundary value problems, Conm. Pure Appl. Math., 18 (1965) 443-492.

[10] Lax, P. D. and Phillips, R. S., Local boundary conditions for dissipative symmetric linear differential operators, Conm. Pure Appl. Math., 13 (1960) 427455.

[11] Lions, J. L. and Magenes, E., Non-homogeneous boundary value problems and applications I, II, III, Grundlehren vol. 181-183 Springer (1972).

[12] Nirenberg, L. and Treves, J. F., On local solvability of linear partial differential equations I and II, Comm. Pure Appl. Math., 23 (1970), 1-38 and 495-510.

[13] Oleinik, O. A. and Radkevich, E. V., On local smoothness of generalied and hypoellipticity of second order differential equations, Uspehi Mat. Nauk 26 (1971) No. 2 (158), 265-281, (Russian Math. Surveys 26 (1971) No. 2, 139-156).

[14] Schechter, M., General boundary value problems for elliptic differential equations, Comm. Pure Appl. Math., 19 (1959) 457-486.

[15] Kannai, Y., Hypoellipticity of certain degenerate elliptic boundary value problems, (to appear).

[16] Taira, K., On the oblique boundary value problems, Proc. Japan Acad. (to appear). 